\title{
POPULATION DYNAMICS OF THREE GAMMARID SPECIES (CRUSTACEA, AMPHIPODA) IN A FRENCH CHALK STREAM
}

\author{
PART II. STANDING CROP
}

\author{
by \\ ANNEMARIE GOEDMAKERS \\ Institute of Taxonomic Zoology, University of Amsterdam, The Netherlands
}

\begin{abstract}
The standing crop of Gammarus pulex pulex (Linnaeus, 1758), G. fossarum Koch in Panzer, 1836 and Ecbinogammarus berilloni (Catta, 1878) has been studied in a small French chalk stream, the Slack. A brief description of all amphipod species encountered in this river is given, with a key to different life stages.

Peaks in abundance could not be explained by population explosions. In spring regions and headwaters population density was highest. The gammarids showed a rather constant zonation in the Slack: $G$. $p$. pulex in the unstable spring region; $G$. fossarum in the upper and middle reaches, confined to the less polluted parts of the latter; and E. berilloni in the middle and lower region of the Slack, where fluctuations in water temperatures increased.

Life histories of all three species revealed great inter- and intraspecific differences. Data on size, growth rate, sexual activity, population structure, sex ratio, fecundity and parasitation yielded ample illustrations of these dissimilarities. Laboratory experiments on egg incubation time and reproduction success demonstrated that temperature affects the three species differently.

Both seasonal variation and between-year differences in life processes were investigated. The impact of temperature was manifestly important in this aspect too.
\end{abstract}

\section{RESUME}

Le «standing crop» de Gammarus pulex pulex (Linnaeus, 1758), G. fossarum Koch in Panzer, 1836, et Echinogammarus berilloni (Catta, 1878) a été étudié dans la Slack, une petite rivière côtière calcaire du Boulonnais. Une courte description est faite de toutes les espèces d'Amphipodes rencontrées dans cette rivière, avec une clef permettant de déterminer les différentes stades de vie.

Les maxima d'abondance ne pouvaient pas être expliqués par des explosions de population. Les densités de population étaient les plus élevées dans les eaux proches de la source. La zonation des Gammares dans la Slack était plutôt constante: G. p. pulex dans les eaux instables proches de la source; $G$. fossarum dans les eaux peu polluées des parties amont et moyen de la rivière; et $E$. berilloni dans les parties moyen et aval de la Slack où les fluctuations de la température de l'eau ont grandies.

Les cycles de vie de toutes les trois espèces montraient des différences importantes inter- et intraspécifiques. Les données recueillies sur la taille, la vitesse de croissance, l'activité sexuelle, la composition des populations, le sex-ratio, la fécondité et le parasitisme livraient d'abondants exemples de ces différences. Des expériences de laboratoire sur le temps d'incubation des oeufs et sur le succès reproducteur montraient des effets de la température différents pour les trois espèces.

L'on a étudié à la fois la variation saisonnière et les différences annuelles des processus de la vie. Là aussi, le facteur de la température se manifestait être d'une grande importance.

\section{CONTENTS}

1. Introduction . . . . . . . . . 32

2. Acknowledgements . . . . . . . . 32

3. Morphology, taxonomy and reproduction . . . 32

1. Morphology . . . . . . . . . 34

2. Diagnosis . . . . . . . . . 34

3. Key . • . • • • • • • • • 34

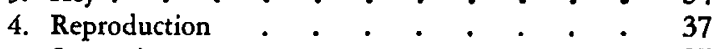

5. Sex ratio . . . . . . . . . . $\quad . \quad 37$

6. Age . . . . . . . . . . . 37

4. Methods . • . . . . . . . 38

5. Results . . . . . . . . . . . 38

1. Population density . . . . . . . 38

2. Microgeographic distribution . . . . $\quad 39$

1. Seasonal and annual variation . . . 39

2. Longitudinal zonation . . . . . 41

3. Reproduction level . . . . . . 42

4. Area shift . . . . . . . . 43

3. Life histories . . . . . . . . . 44

1. Growth and size .. . . . . . 47

1. Interspecific differences . . . . 48

2. Microgeographic variation . . . . 48

3. Seasonal and annual variation . . . 52

2. Sexual activity . . . . . . . 53

1. Interspecific differences . . . . 54

2. Microgeographic variation . . . $\quad 55$

3. Seasonal and annual variation . . . 56

3. Sex ratio . . . . . . . . 56

1. Interspecific differences . . . . 57

2. Microgeographic variation . . . $\quad .57$

3. Seasonal and annual variation . . . 58

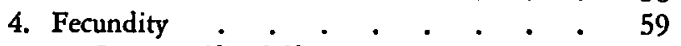

1. Interspecific differences . . . . 59

2. Microgeographic variation . . . . 60

3. Seasonal and annual variation. . . 61 
5. Condition . . . . . . . . 63

1. Parasitation . . . . . . . 63

2. Damaged animals . . . . . . 64

6. Death . . . . . . . . . 64

4. Laboratory experiments . . . . . 64

1. Egg incubation time . . . . . . 64

2. Reproductive success . . . . . . . 65

5. Calculated life histories . . . . . 65

6. Environmental conditions . . . . . . 66

6. Conclusions . . . . . . . . . . 67

References . . . . . . . . . 68

\section{INTRODUCTION}

Clemens (1950) initiated the detailed study of life histories of gammarids in the field with a description of the life cycle of Gammarus fasciatus Say, 1818. Much work has been done afterwards on two European freshwater species Gammarus pulex pulex (Linnaeus, 1758) and G. fossarum Koch in Panzer, 1836 (Hynes, 1955; Macan \& Mackereth, 1957; Maitland, 1966; Lehmann, 1967; Roux, 1970; Hultin, 1971; Obrdlík, 1972; Van den Beld, 1973; Sukop, 1975; Teckelmann, 1974; Iversen \& Jessen, 1977; Pieper, 1978; and Welton, 1979). These closely related species (Pinkster, 1972) are two of the gammarid species living in the limnic part of the river Slack (Pasde-Calais, France). The other freshwater gammarid living in this stream is Echinogammarus berilloni (Catta, 1878). Although many authors published on its distribution, little is known about its biology (Vincent, 1971; Pinkster, 1973).

Studies on life histories often compare different species or populations of one species inhabiting clearly different habitats (Hynes, 1955; Roux, 1970; Meijering \& Teichmann, 1978). It is commonly believed that different populations of one species living under broadly the same circumstances have largely identical life cycles.

The present study is based on samples of the gammarid population collected at different stations in the river Slack at certain time intervals. The aim of this standing crop research was manifold: - to determine the distribution patterns of the gammarid species living in this stream;

- to check whether these patterns are stable or liable to changes during the seasons and the years;

- to supplement our knowledge of E. berilloni;
- to investigate whether life cycles of different populations of the same species are alike throughout this relatively small river.

These standing crop data were related to changes and fluctuations in environmental factors (Goedmakers, 1980).

The present study represents part II of a research program published in four parts: part I, general aspects and environmental factors (Goedmakers, 1980); part III, migration research (Goedmakers \& Pinkster, in press); part IV, review and implications.

With this program we wanted to solve the question when, how, and why freshwater gammarids realize short term fluctuations in their microgeographic distribution area. Our research methods enabled us to give a qualitative impression of biological processes in a small chalk river.

\section{ACKNOWLEDGEMENTS}

Besides Drs. Cita Vissers and Drs. Joost Teixeira de Mattos, with whom I worked on the material we collected together in the field, I would like to thank Mr. Paul Tels, Mrs. Mieke van der Ven, Drs. Nico Broodbakker and Mr. Bart van der Hoek for their assistance with the tedious and monotonous treatment of the standing crop samples. Mrs. Karin Ravenstein, Mrs. Heleen Blom, and Mr. Peter Huls worked with me on the fecundity of the three gammarid species. Thanks to the painstaking care of Mr. Pero Versteeg and identifications and measurements by Mr. Peter Finger it was possible to do some laboratory experiments. The vast mass of data could only be mastered by the grace of the mathematical help of Drs. Jan Dieleman and Mr. Freek Burger. All are allied to the Institute of Taxonomic Zoology of the University of Amsterdam, except Mr. Burger, of the Mathematical Centre in Amsterdam.

This work has been done with financial support of the Netherlands Organization for the Advancement of Pure Research (ZWO), the Foundation for Fundamental Biological Research (BION), and the University of Amsterdam.

\section{MORPHOLOGY, TAXONOMY AND REPRODUCTION}

Most taxonomic work on gammarids is rather inaccessible to ecologists. Two reasons for this may be mentioned: firstly, the preference of the taxonomist to cover wide geographical areas, thereby including rare and uncommon taxa; secondly, the emphasis put on characteristics of male adults. Most morphological characters, however, are widely variable and show considerable changes 


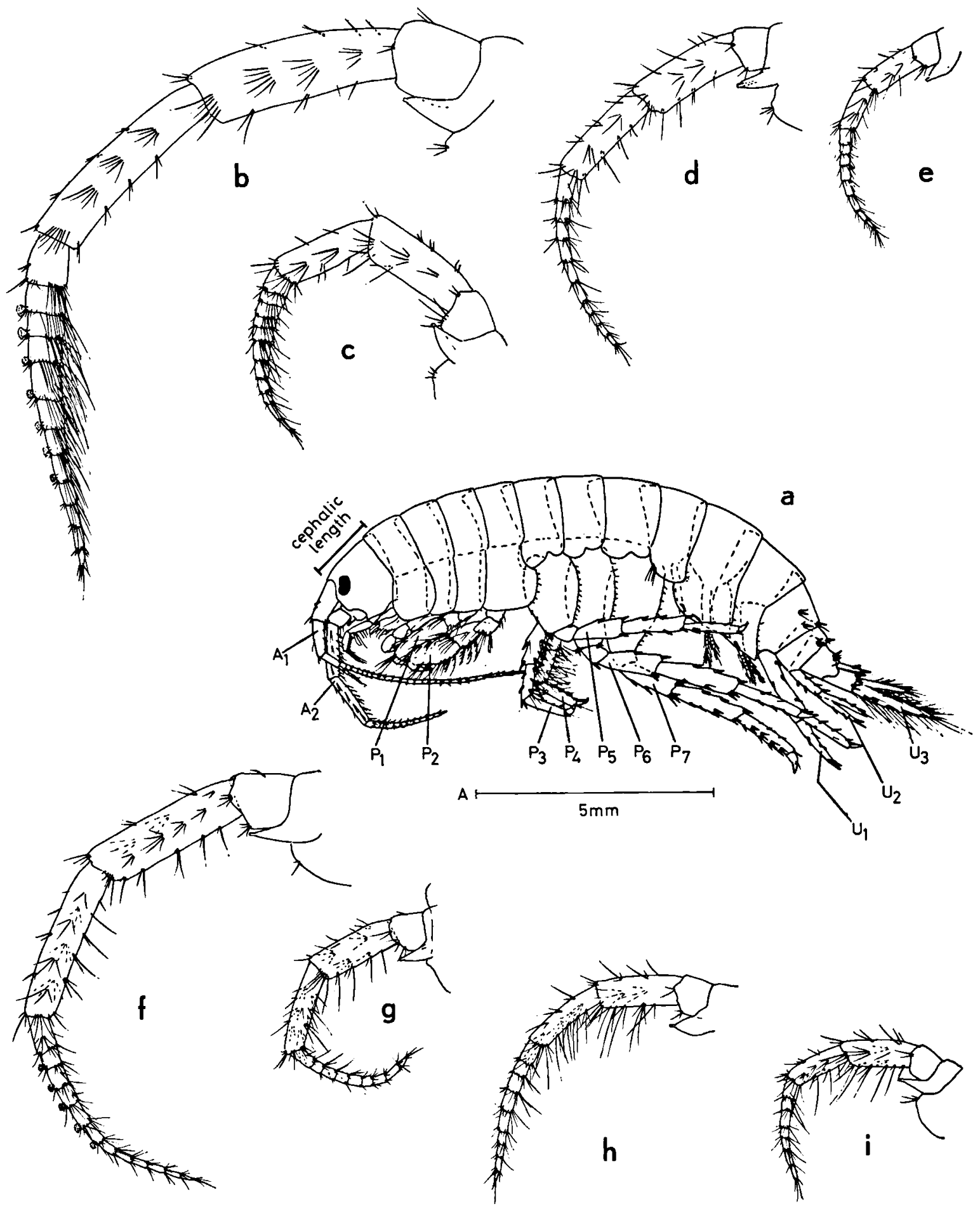

Fig. 1. a, Gammarus fossarum, habitus adult $\hat{\delta}$ (scale $\mathbf{A} ; \mathbf{A}=$ antenna, $\mathbf{P}=$ pereiopod, $\mathrm{U}=$ uropod). b-e, Gammarus pulex pulex second antenna: b, adult $\hat{o} ; c$, subadult $\hat{\delta}$; d, ovigerous $q$; e, subadult $q$ (scale C). $\mathrm{f}-\mathrm{i}$, Gammarus fossarum second antenna: $\mathrm{f}$, adult $\hat{\delta} ; \mathrm{g}$, subadult $\hat{o} ; \mathrm{h}$, ovigerous $q$; $\mathrm{i}$, subadult $q$ (scale $\mathrm{C}$ ). 
during development. Therefore, sometimes characters of juveniles or females of one species can be identical to those of adult males of a different species. Comparing males, females and juveniles simultaneously would make matters very complicated for a taxonomist working on many taxa at the same time. But, on the other hand, ecologists working in a limited area need identification characters for all developmental stages of the small number of species they investigate.

$G$. p. pulex, G. fossarum and E. berilloni occur frequently in western Europe and are the subject of much ecological research. This chapter, without being exhaustive, is meant to offer a guide for ecologists working with the same species. Further details on taxonomy are given by Pinkster (1973), Karaman \& Pinkster (1977) and Sexton (1942). For detailed information on anatomy and reproduction of gammarids, Heinze (1932) and Le Roux (1933) should be consulted.

\subsection{Morphology}

Gammarids have two eyes, two pairs of antennae (A1 and A2), a series of mouth parts, seven pairs of walking legs or pereiopods (P1 to P7) of which the first two have a prehensile function (gnathopods), three pairs of swimming legs or pleopods, three pairs of uropods (U1 to U3) and an unpaired telson (fig. 1a). Especially the shape of the eyes, the setation of the second antenna and the shape of the third uropod are useful features for the identification of the species that are found in the freshwater part of the river Slack.

Sex can be determined before sexual maturity is reached because females have pairs of oostegites on P2 to P5 (figs. 3h and i) and males two genital papillae on P7 (fig. 3j). Animals without these features are considered to be juveniles. Males become larger than females.

\subsection{Dia gnos is}

Several Crustacea can be found in a river like the Slack. The most important among them are isopods and amphipods. A short list with a diagnosis of the various amphipod species encountered might be convenient for ecologists working on the same species.
Niphargus sp.: blind, unpigmented species, occurring occasionally in springs or springbrooks of the Slack (not illustrated here).

Gammarus pulex pulex (Linnaeus, 1758): a rather robust-looking species with round to ovoid eyes; short setae on peduncle segments 4 and 5 of A2, distally increasing in length; a long inner ramus of $\mathrm{U} 3$ (figs. $1 \mathrm{~b}-\mathrm{e}, 2 \mathrm{i}-\mathrm{l}, 3 \mathrm{c}-\mathrm{d}$ ).

Gammarus fossarum Koch in Panzer, 1836: a more slender-looking species with round to ovoid eyes; long setae on peduncle segments 4 and 5 of A2; a short to medium long inner ramus of U3 (figs. 1a, f-i, $2 \mathrm{~m}-\mathrm{q}, 3 \mathrm{e}-\mathrm{j}$ ).

Echinogammarus berilloni (Catta, 1878): a slender-looking species with elongate eyes; setae of variable length on peduncle segments 4 and 5 of A2; a very short, reduced inner ramus of $\mathrm{U} 3$ (figs. $2 \mathrm{a}-\mathrm{h}, 3 \mathrm{a}-\mathrm{b}$ ).

Gammarus zaddachi Sexton, 1912: a brackishwater species, migrating into the limnic part of the river, with elongate eyes; long setae on peduncle segments 4 and 5 of A2; a long inner ramus of U3 (not illustrated here).

\subsection{K e y}

Since characteristics of e.g. females of one species may resemble those of males of another species (compare figs. 21 and $2 \mathrm{~m}-\mathrm{n}$ or $1 \mathrm{f}$ and $2 \mathrm{~b}$ ) and because juveniles have not yet developed the same characteristics as adults, we made a key to the various sexes and life stages of the five amphipod species living in the limnic part of the river Slack.

1 a. Eyes round to ovoid, upper margin widely separated from middorsal line; inner ramus of $U_{3}$ at least $1 / 3$ of first segment of outer ramus . . . . 3

b. Eyes elongate, reniform, upper margin near to middorsal line . . . . . . . . . . . 2

c. Eyes absent . . . . . . . Niphargus sp.

2 a. Inner ramus of $U_{3}$ at least half as long as first segment of outer ramus. (In 3 is shown how the sexes can be distinguished.) . . . . . . G. zaddachi

b. Inner ramus of $\mathrm{U} 3$ very short, reduced. (In 3 is shown how the sexes can be distinguished.) . E. berilloni

3 a. Genital papillae present ( \&) . . . . 4

b. Oostegites present ( $\%$ ). . . . . . . 5

c. No genital papillae or oostegites present (juvenile) 6

4 a. At least 4 tufts of equally long setae, implanted at regular intervals on peduncle segments 4 and 5 of A2, each as long as or longer than the diameter of these segments; inner ramus of U3 $1 / 2$ to $2 / 3$ of first segment of outer ramus . . . . G. fossarum

b. No more than 4 tufts of setae, implanted at irregular 


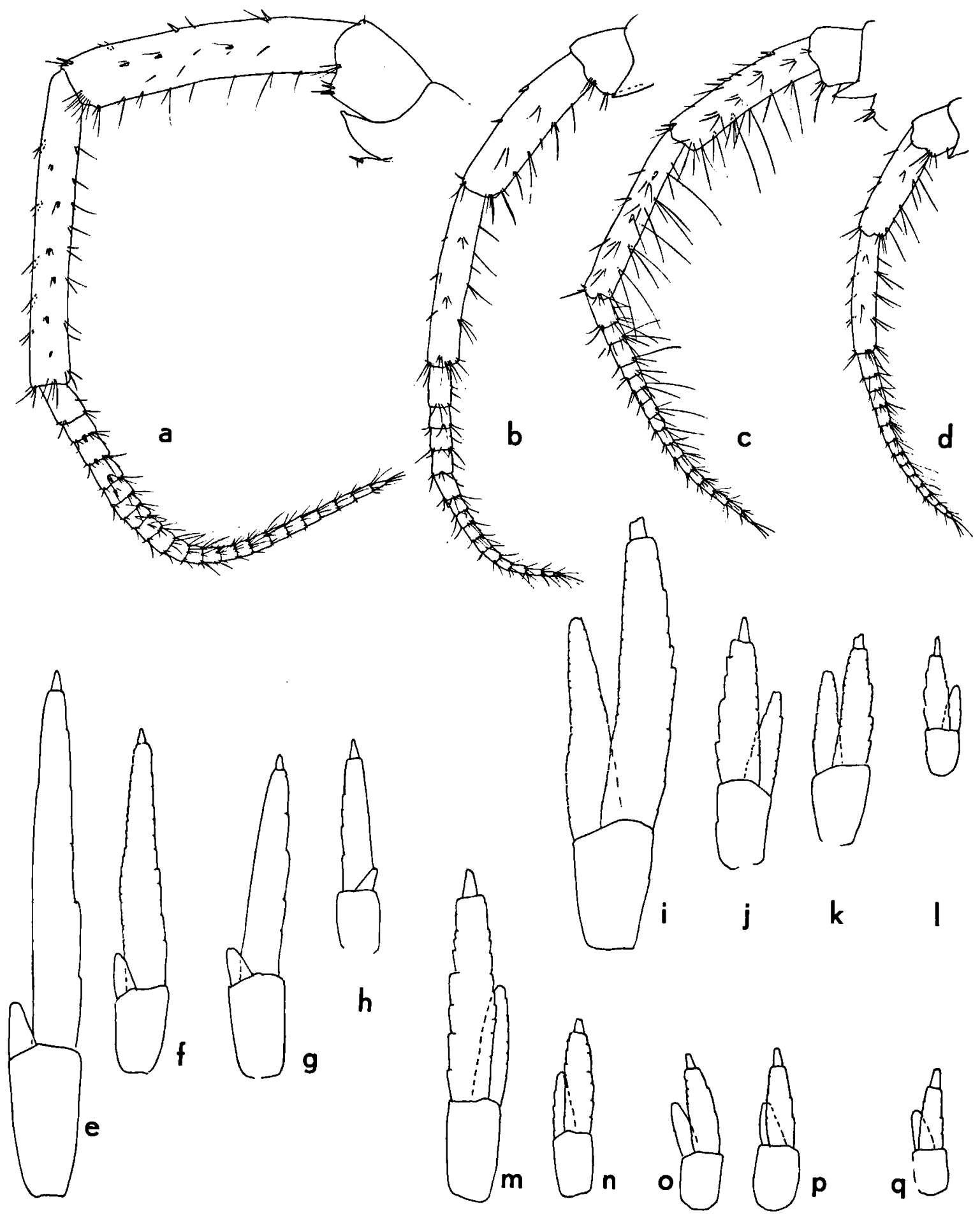

Fig. 2. a-d, Echinogammarus berilloni second antenna: a, adult $\hat{\delta} ; b$, subadult $\hat{o}$; c, ovigerous $q$; d, subadult $\$$. e-h, Echinogammarus berilloni third uropod: $e$, adult $\hat{o} ; \mathrm{f}$, subadult $\hat{o} ; \mathrm{g}$, ovigerous $q ; \mathrm{h}$, subadult $q$. $\mathrm{i}-1$, Gammarus pulex pulex third uropod: $\mathrm{i}$, adult $\hat{\delta} ; \mathrm{j}$, subadult $\hat{\delta} ; \mathrm{k}$, ovigerous $q ; \mathrm{l}$, subadult $q$. $\mathrm{m}-\mathrm{q}$, Gammarus fossarum third uropod: $\mathrm{m}$, adult $\hat{\delta} ; \mathrm{n}$, subadult $\hat{\delta}$; $\mathrm{o}$ and $\mathrm{p}$, ovigerous $q$; $\mathrm{q}$, subadult $q$. (All scale $\mathrm{C}$.) 


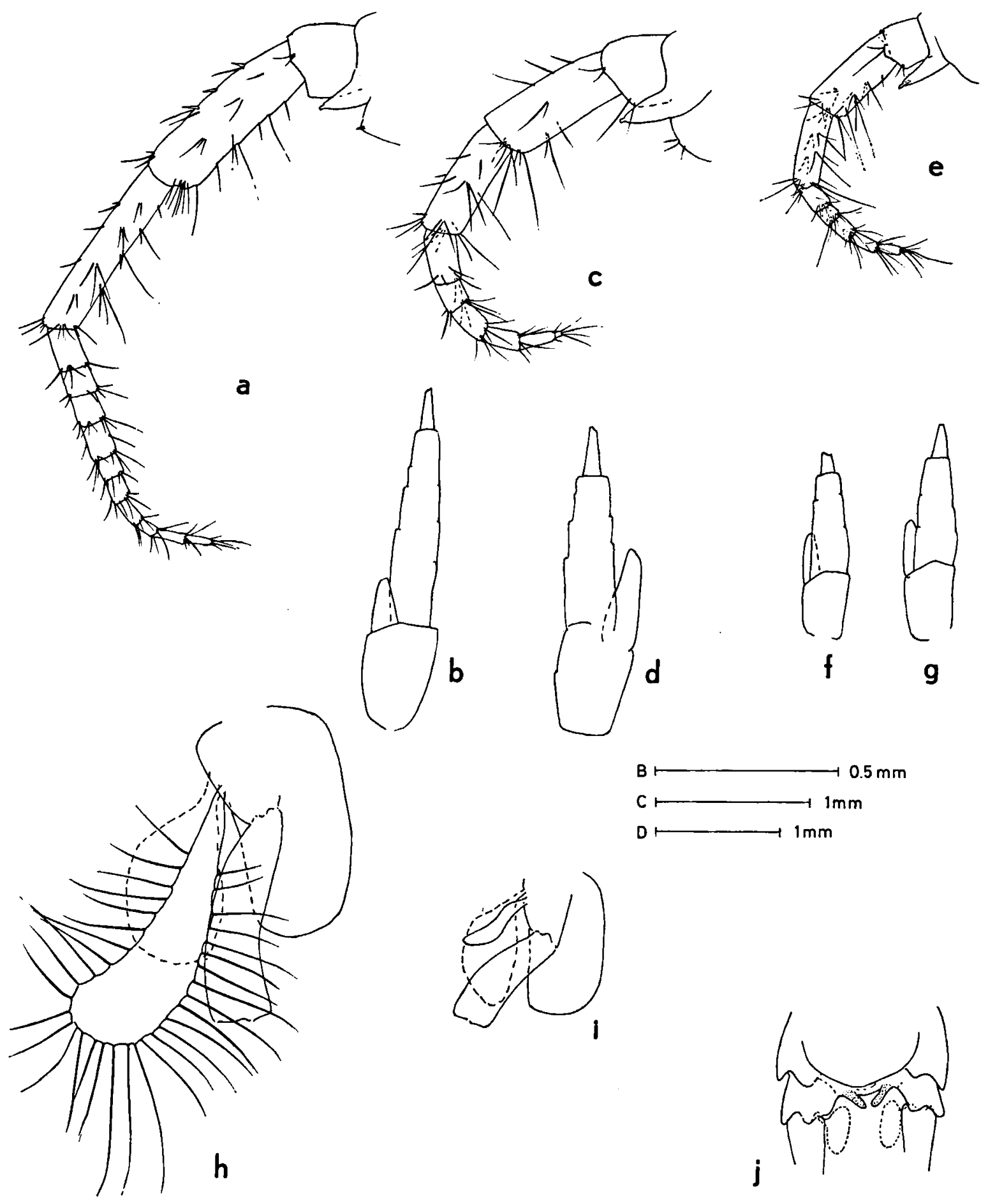

Fig. 3. a-b, Echinogammarus berilloni juvenile: a, second antenna; b, third uropod.

c-d, Gammarus pulex pulex juvenile: c, second antenna; d, third uropod.

e-j, Gammarus fossarum: e, second antenna juvenile; $f$ and $g$, third uropod juvenile; $h$, oostegites third pereiopod ovigerous $q$; i, oostegites third pereiopod subadult $\$ ; j$, genital papillae. (Juveniles scale B, other figures scale D.) 
intervals on peduncle segments 4 and 5 of $A 2$; the length of these setae increases towards the distal end of the segments, but is always as long as or shorter than their diameter; inner ramus of U3 $2 / 3$ to $3 / 4$ of first segment of outer ramus . . . . G.p.pulex

5 a. At least 4 tufts of equally long setae, implanted at regular intervals on peduncle segments 4 and 5 of A2, always longer than the diameter of these segments; inner ramus of U3 $1 / 2$ to $2 / 3$ of first segment of outer ramus . . . . . . . G. fossarum

b. No more than 4 tufts of setae, implanted at irregular intervals on peduncle segments 4 and 5 of $A 2$, increasing in length towards the distal end of these segments, but never longer than the diameter of them; inner ramus of $U 332 / 3$ to $3 / 4$ of first segment of outer ramus. . . . . . . G.p.pulex

6 a. Inner ramus of $U 31 / 3$ to $1 / 2$ of first segment of outer ramus; 3 or more tufts of setae on peduncle segments 4 and 5 of A2 . . . . . G. fossarum

b. Inner ramus of $U 3$ at least $1 / 2$ of first segment of outer ramus; 3 or less tufts of setae implanted almost entirely near the distal end of peduncle segments 4 and 5 of $\mathrm{A2}$.

G. p. pulex

Although differences between the two species $G$. fossarum and $G$. p. pulex are based on the same features in all developmental stages, at times the accent lies on the relative length of the inner ramus of $\mathrm{U} 3$ and at other times on the setation of A2. The most important character is always mentioned first in the key. The best way to discriminate between these two species is comparing the figures with the animals at hand. Especially for very young or damaged animals some uncertainty may remain.

Some characteristics, like the presence of a medial palmar spine on the gnathopods of males of both $G$. fossarum and $G$. p. pulex and the presence of curled setae on the urosome of males of $E$. berilloni are handy means of identifying large animals of these species. However, since these features can not always be used for small animals, I did not include them in the key.

\subsection{Reproduction}

Some time before a female deposits her eggs, the male grasps her. Together they swim around in precopulation until the female moults and can be fertilized. The duration of this precopulation is largely dependent on temperature and lasts from some hours to some weeks. The female lays her eggs in a broodpouch formed by the four pairs of oostegites, where they remain until hatching. Hatched juveniles may also stay in the brood- pouch for some days, especially if conditions are unfavourable.

We used the mean percentage of precopulations and that of ovigerous females as measures for the sexual activity of a given population at a certain time.

The number of eggs a female carries in her broodpouch is called fecundity. Fecundity is dependent on the species concerned and varies with age and environmental conditions.

The eggs develop through several stages (fig. $4)$. In the process part of the eggs may get lost, so the number of offspring a female produces does not necessarily equal the number of eggs she carried initially.

\subsection{Sex ratio}

The number of males divided by the number of females is called sex ratio. This ratio may depend on environmental conditions, on population structure, and on the species concerned.

\subsection{A ge}

Gammarids frequently moult and are able to grow, sometimes considerably, with every moult. Since their body is comma-shaped, their total body length is not a very convenient standard to judge their age. Their cephalic length on the other hand is rather easy to determine and was therefore used as an age indicator (cf. Dennert et al., 1969).
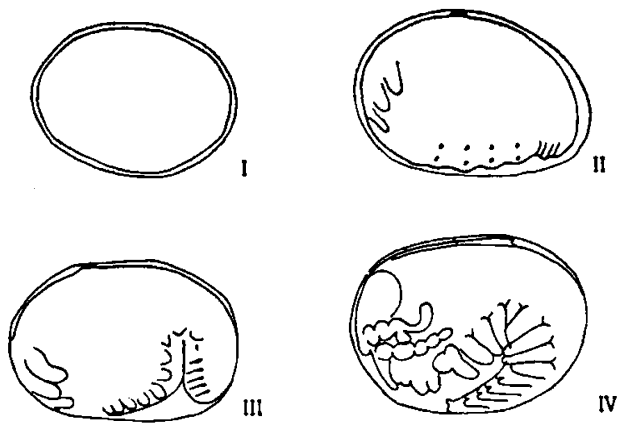

Fig. 4. Egg developmental stages.

I: The stage between fertilization and the initiation of the antennae.

II: Initiation of the antennae visible.

III: Comma-shape and the initiation of the pereiopods visible.

IV: Clearly visible constriction of the comma; appendages are segmented and make a slender impression.

V: Juveniles completely developed (not figured). 


\section{METHODS}

The way we sampled the standing crop at different stations in the river Slack by electrofishing or kicksampling with a hand net is described in detail in part I of this study (Goedmakers, 1980). Data on electrofishing, the quantitative but time-consuming way of sampling standing crop, have contributed to our results only incidentally. As a rule we investigated the standing crop by kicksampling. In this method the area upstream of a hand net is vigorously disturbed with a foot and animals are swept into the hand net by the current (Hynes, 1961; Frost et al., 1971). The number of ten second scoops necessary to catch one hundred animals was counted and used as an indicator of population density (see also Armitage et al., 1974).

A standing crop sample of about a hundred gammarids was stored and identified, sexed and measured in the laboratory. Different features of a population like sexual activity, fecundity and sex ratio could thus be determined.

Some laboratory experiments concerned the developmental time and reproductive success of our gammarid species. The animals (100 or 200) were kept together in plastic trays $(25 \times 25 \mathrm{~cm}$; 6.251 of water) under different temperature conditions. Ovigerous females found in these basins were put apart, each in its own small basin $(7 \times 7 \mathrm{~cm} ; 0.491$ of water) until juveniles hatched. Juveniles were then counted and reared apart from their mother until they reached sexual maturity (for details see Goedmakers, 1972). Animals collected at stations 1,5 and 10 on 14 October 1975 were used. ${ }^{1}$ )

The mathematical treatment of our material was done with the CDC computer of SARA (Stichting Academisch Rekencentrum Amsterdam).

\section{RESULTS}

Neither by electrofishing nor by hand-net fishing all categories of the standing crop can be sampled representatively. Juveniles for instance, living most of the time hidden in the substrate, are captured

1) For a description of all sampling stations see Goedmakers (1980). in relatively low percentages with both methods, although electrofishing samples tend to contain more juveniles (Goedmakers, 1980; De Weerd, unpubl.). We took great care to take samples of the standing crop in a constant and identical way throughout our programme. Thus, standing crop samples may be compared, even if they are not a completely true representation of the population living at a given locality. This aspect emphasizes the qualitative character of our research project.

\subsection{Population density}

The results of electrofishing in July 1975 indicate the absolute population density (Meijering, 1972) in numbers of gammarids at the different sampling stations (table I). At most stations, the population density in the middle of the stream near the drift nets is either larger or of the same order as that near the banks of the river. Population densities vary from 500 to 10,000 gammarids $\cdot \mathrm{m}^{-2}$. Spring regions and headwaters showed relatively high population densities.

The second way in which we estimated the population density - by counting the number of

\section{TABLE I}

Population density of gammarids measured by electrofishing in numbers $\mathrm{m}^{-2}$ or estimated by kick-sampling in numbers of ten second scoops, necessary to catch one hundred animals (29 June-6 July 1975).

\begin{tabular}{|c|c|c|c|}
\hline & \multicolumn{2}{|c|}{ electrofishing } & \multirow{2}{*}{$\begin{array}{c}\text { kick- } \\
\text { sampling }\end{array}$} \\
\hline & bank & middle & \\
\hline Sta. 1 & \multicolumn{2}{|c|}{$2595-3750 *$} & 1 \\
\hline Sta. 2 & 1465 & 7875 & 2 \\
\hline Sta. $2 \mathrm{a}$ & \multicolumn{2}{|c|}{$4800-9825 *$} & 4 \\
\hline Sta. 3 & 6110 & 3280 & 1 \\
\hline Sta. 4 & $1390-9300$ & 8700 & 1 \\
\hline Sta. 5 & 3910 & 4200 & 1 \\
\hline Sta. 6 & 1405 & 1250 & 2 \\
\hline Sta. 7 & 910 & 3725 & 1 \\
\hline Sta. 8 & 1170 & 525 & 7 \\
\hline Sta. 9 & 1115 & 6200 & 2 \\
\hline Sta. 10 & 2800 & $4375-8590$ & 1 \\
\hline Sta. 11 & 400 & 1140 & 1 \\
\hline Sta. 12 & 900 & 1840 & 5 \\
\hline
\end{tabular}

* At these stations the river is too narrow to differentiate between bank and middle of the stream with our electrofishing method. 
TABLE II

Population density of gammarids estimated by kick-sampling in numbers of ten second scoops, necessary to collect a sample of one hundred animals (November 1974-September 1975). Maximal population densities are printed in bold type.

\begin{tabular}{|c|c|c|c|c|c|c|c|c|c|c|c|}
\hline & $\begin{array}{l}\mathrm{N} \\
1974\end{array}$ & $\mathrm{D}$ & $\begin{array}{l}\mathrm{J} \\
1975\end{array}$ & $\mathbf{F}$ & $\mathbf{M}$ & A & $\mathbf{M}$ & $\mathrm{J}$ & $\mathbf{J}$ & A & $\mathrm{S}$ \\
\hline $\begin{array}{ll}\text { Sta. } & 1 \\
\text { Sta. } & 2 \\
\text { Sta. } & 2 a \\
\text { Sta. } & 3 \\
\text { Sta. } & 4 \\
\text { Sta. } & 5 \\
\text { Sta. } & 6 \\
\text { Sta. } & 7 \\
\text { Sta. } & 8 \\
\text { Sta. } & 9 \\
\text { Sta. } & 10 \\
\text { Sta. } & 11 \\
\text { Sta. } & 12\end{array}$ & $\begin{array}{r}4 \\
6 \\
2 \\
3 \\
2 \\
7 \\
2 \\
2 \\
6 \\
5 \\
4 \\
10 \\
6\end{array}$ & $\begin{array}{l}5 \\
6 \\
2 \\
3 \\
1 \\
6 \\
2 \\
5 \\
1.5 \\
9 \\
4 \\
8 \\
11\end{array}$ & $\begin{array}{l}2 \\
4 \\
1 \\
3 \\
1 \\
1 \\
5 \\
3 \\
5 \\
2 \\
2 \\
5 \\
4\end{array}$ & $\begin{array}{l}2 \\
2 \\
1 \\
2 \\
1 \\
3 \\
3 \\
5 \\
5 \\
4 \\
3 \\
8 \\
8\end{array}$ & $\begin{array}{r}1 \\
2 \\
1 \\
3 \\
2 \\
2 \\
3 \\
3 \\
6 \\
2 \\
3 \\
10 \\
5\end{array}$ & $\begin{array}{l}2 \\
1 \\
2 \\
4 \\
2 \\
2 \\
3 \\
6 \\
5 \\
4 \\
4 \\
9 \\
5\end{array}$ & $\begin{array}{l}2 \\
3 \\
1 \\
3 \\
2 \\
3 \\
2 \\
3 \\
7 \\
2 \\
2 \\
3 \\
5\end{array}$ & $\begin{array}{l}2 \\
1 \\
2 \\
1 \\
2 \\
2 \\
2 \\
2 \\
4 \\
3 \\
2 \\
4 \\
4\end{array}$ & $\begin{array}{l}1 \\
2 \\
4 \\
1 \\
1 \\
1 \\
2 \\
1 \\
7 \\
2 \\
1 \\
1\end{array}$ & $\begin{array}{l}3 \\
2 \\
2 \\
1 \\
3 \\
2 \\
3 \\
1 \\
8 \\
2 \\
2 \\
2 \\
2\end{array}$ & $\begin{array}{l}4 \\
3 \\
3 \\
2 \\
1 \\
1 \\
2 \\
1 \\
4 \\
2 \\
1 \\
3 \\
4\end{array}$ \\
\hline $\begin{array}{l}\text { number of times } \\
\text { population density } \\
\text { reaches a maximum }\end{array}$ & 1 & 3 & 4 & 2 & 3 & 1 & 3 & 3 & 9 & 4 & 6 \\
\hline
\end{tabular}

ten second scoops needed to catch one hundred animals - is a comparative method. Since it is much more difficult to catch animals in places with a rich aquatic vegetation or a silty substrate than at stations with gravel, one can not ascertain absolute population numbers with this method. This means that stations with a different substrate structure can not be compared adequately (table I). The method can be used, however, as a quick way to measure fluctuations in population density at a given station throughout the year and gives a rough estimate of the range of fluctuations (table II).

Highest population densities were usually found in summer. In 9 out of 13 stations population density reached a maximum value in July. Throughout the year, population densities at one station may differ by a factor 10 , although this figure might be too high due to the fact that gammarids are more easily captured when they are active. Gammarids become more active as temperatures rise. Therefore the high population densities found during summer could have been partly caused by a relatively high activity of the animals in that period.
5.2. Microgeographic distribution After determining the species composition of each standing crop sample, the percentage of each gammarid species living at a given locality at a certain time could be compared. It is important to realize that in this way we compare relative abundances. Only if we would have used standing crop data in which population density is taken into account (resulting in real abundance data), we could have avoided this. However, this approach would have meant either a totally different sampling program or, when using the present sampling program, the introduction of other possible errors (see section 5.1). As a comparison of relative abundances did not interfere with our conclusions, we contented ourselves with this way of comparing samples. In doing so one has to remain aware that for instance an increase in percentage of a certain species may either indicate an increase in numbers of that very species and/or a decrease in numbers of other species living at the same locality.

\subsubsection{Seasonal and annual variation}

Station 1 (figs. 5 and 6) has a fluctuating percentage of G.p.pulex and $G$. fossarum throughout 


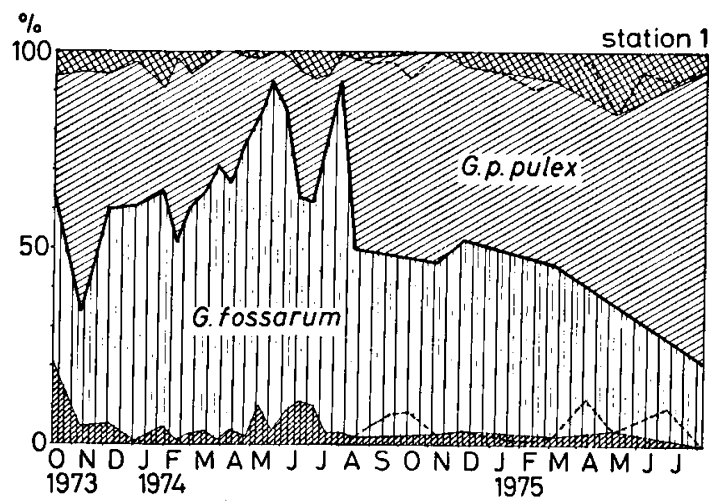

Fig. 5. Seasonal variation in the distribution of $G$. fossarum and $G$. p. pulex with the seasonal variation in the occurrence of ovigerous females (chequered parts) of both species at station 1. (The broken lines give the percentages of ovigerous females for samples in which only ovigerous females were identified. Sample size varies from $n=88$ to $n=263$.)

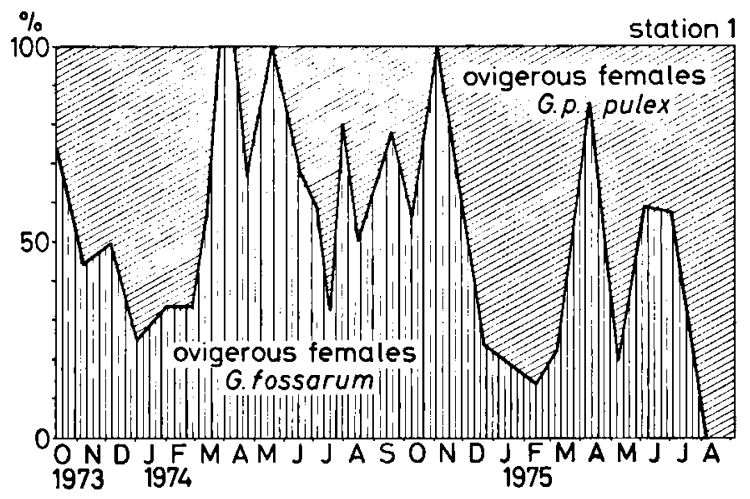

Fig. 6. Seasonal variation in the distribution of ovigerous females only of G. fossarum and G. p. pulex at station 1. (Sample size varies from $n=1$ to $n=71$.)

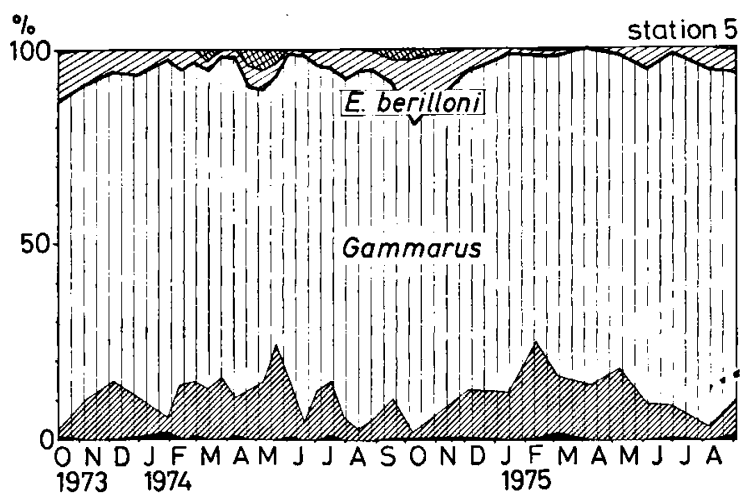

Fig. 7. Seasonal variation in the distribution of Gammarus and $E$. berilloni with the seasonal variation in the occurrence of ovigerous females of G. p. pulex (solid black), G. fossarum (chequered) and $E$. berilloni (chequered), at station 5 . (Sample size varies from $n=96$ to $n=236$ ).

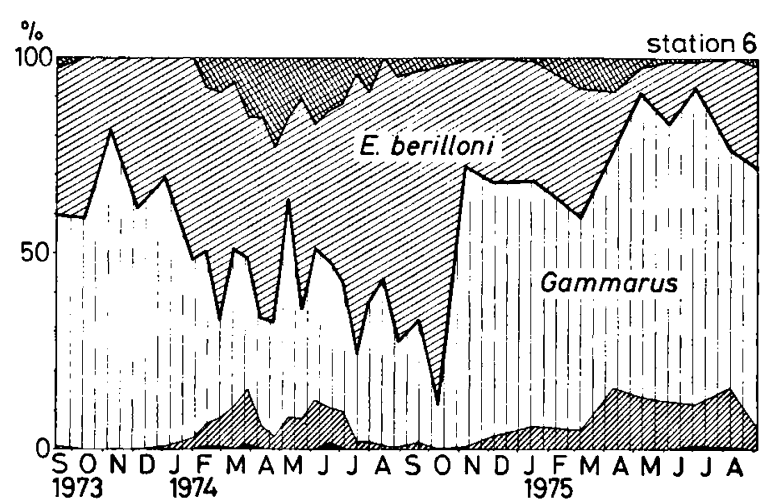

Fig. 8. Seasonal variation in the distribution of Gammarus and $E$. berilloni with the seasonal variation in the occurrence of ovigerous females of G. p. pulex (solid black), G. fossarum (chequered) and $E$. berilloni (chequered), at station 6 . (Sample size varies from $n=89$ to $n=239$.)

the year. During the first half of the sampling period $G$. fossarum enlarged its share of the total gammarid population, while in 1975 G. p. pulex occurred in increasing percentages. ${ }^{2}$ ) Both the population as a whole and the ovigerous females only tend to consist of initially increasing and later decreasing percentages of $G$. fossarum, although this phenomenon is less pronounced in data of ovigerous females only (fig. 6). This difference is due to the distinct reproduction periods of the two species (see section 5.3.2) at this station.

Stations 2, 2a, 3 and 4 are mainly inhabited by $G$. fossarum (table III). Here, G. p. pulex forms a rather constant but very small part of the gammarid population. Every now and then a small number of $E$. berilloni is found at station 3 .

At stations 5 to $9 G$. fossarum and $E$. berilloni coexist in fluctuating percentages, whereas $G$. $p$. pulex constitutes a similar, small part of the gammarid population as at stations 2 to 4 (table III). Stations 5 and 6 (figs. 7 and 8) show peaks in the percentage of $E$. berilloni in the fall of 1974 , followed by a steep rise in the percentage of $G$. fossarum. At station 7 (fig. 9) the percentage of $G$. fossarum steadily increased till May 1975 ,

2) Since the sorting out of G. fossarum and G. p. pulex is a very time-consuming procedure, sometimes only the ovigerous females were identified. The solid lines in fig. 5 connect data of samples that were fully identified; the broken lines those of which only ovigerous females were identified. 
TABLE III

Microgeographic distribution of G. p. pulex, G. fossarum, E. berilloni and G. zaddachi. Mean species composition at the different sampling stations (only fully identified samples of entire sampling period averaged).

\begin{tabular}{|c|c|c|c|c|c|c|c|c|c|}
\hline & \multirow{2}{*}{$\begin{array}{l}\text { number } \\
\text { of } \\
\text { samples }\end{array}$} & \multicolumn{2}{|c|}{ G. p. pulex } & \multicolumn{2}{|c|}{ G. fossarum } & \multicolumn{2}{|c|}{ E. berilloni } & \multicolumn{2}{|c|}{ G. zaddacbi } \\
\hline & & mean $\%$ & SD & mean $\%$ & $\mathrm{SD}$ & mean $\%$ & $\mathrm{SD}$ & mean $\%$ & $\mathrm{SD}$ \\
\hline Sta. 1 & 24 & 38.3 & 17.9 & 61.7 & 17.9 & 0.0 & 0.0 & 0.0 & 0.0 \\
\hline Sta. 2 & 19 & 0.9 & 1.1 & 99.0 & 1.1 & 0.1 & 0.3 & 0.0 & 0.0 \\
\hline Sta. $2 a$ & 14 & 2.7 & 2.5 & 97.3 & 2.5 & 0.0 & 0.0 & 0.0 & 0.0 \\
\hline Sta. 3 & 21 & 0.8 & 1.1 & 97.6 & 2.9 & 1.6 & 2.9 & 0.0 & 0.0 \\
\hline Sta. 4 & 20 & 1.8 & 1.5 & 98.0 & 1.7 & 0.2 & 0.4 & 0.0 & 0.0 \\
\hline Sta. 5 & 20 & 2.1 & 2.3 & 92.9 & 4.1 & 5.0 & 3.2 & 0.0 & 0.0 \\
\hline Sta. 6 & 20 & 2.7 & 3.2 & 52.9 & 16.1 & 44.4 & 16.7 & 0.0 & 0.0 \\
\hline Sta. 7 & 19 & 1.3 & 1.7 & 47.8 & 16.9 & 50.9 & 17.1 & 0.0 & 0.0 \\
\hline Sta. 8 & 30 & 1.4 & 1.3 & 40.6 & 14.4 & 58.0 & 14.6 & 0.0 & 0.0 \\
\hline Sta. 9 & 21 & 2.6 & 2.3 & 91.2 & 7.1 & 6.2 & 5.7 & 0.0 & 0.0 \\
\hline Sta. 10 & 21 & 0.4 & 1.2 & 3.7 & 3.9 & 95.9 & 4.1 & 0.0 & 0.0 \\
\hline Sta. 11 & 19 & 0.0 & 0.0 & 2.1 & 2.7 & 97.9 & 2.7 & 0.0 & 0.0 \\
\hline Sta. 12 & 21 & 0.5 & 1.2 & 1.8 & 3.8 & 97.7 & 4.7 & 0.0 & 0.0 \\
\hline Sta. 13 & 9 & 0.4 & 0.6 & 1.9 & 2.5 & 97.7 & 2.6 & 0.0 & 0.0 \\
\hline Sta. 14 & 9 & 11.9 & 17.3 & 6.7 & 15.9 & 66.5 & 26.8 & 14.9 & 27.9 \\
\hline Sta. 15 & 10 & 0.0 & 0.0 & 0.0 & 0.0 & 8.2 & 25.2 & 91.8 & 25.2 \\
\hline
\end{tabular}

but rapidly dropped in summer. At station 8 (fig. 10) E. berilloni slowly but constantly enlarged its share throughout the sampling period. At station 9 (fig. 11) a considerable increase in the percentage of $E$. berilloni during the second half of the sampling period was found.

The populations at stations 10 to 13 consisted mainly of $E$. berilloni (table III). G. fossarum was always present, although in limited percentages, whereas $G$. p. pulex was only sporadically found. In the period from mid-July to the end of 1974, after a severe case of oil pollution, no gammarids at all could be found at station 13 .

Station 14, in the freshwater tidal area, is invaded by $G$. zaddachi during certain periods of the year (Dennert et al., 1969), but ovigerous females of this species have never been found here. G. p. pulex occurs in relatively high percentages as compared with the other stations in this river. Again, G. fossarum forms a constant but small portion of the population, whereas $E$. berilloni is by far the dominating species. Data on seasonal variation are less significant as they are based on the often very small numbers of individuals that could be found.

Stations 15 , in the brackish tidal area, is most of the time mainly inhabited by $G$. zaddacbi, but nearly always low percentages of $E$. berilloni were found. At this station, population densities can be very low as well.

Apparently, no regular seasonal fluctuations in the species composition of the gammarid populations at the various stations were found (except for G. zaddachi at station 14, which is situated outside our real research area).

\subsubsection{Longitudinal zonation}

Although the percentage of each gammarid species at a certain locality may change during the year, as is shown above, the longitudinal zonation of the three limnic species remained rather constant between September 1973 and September 1975. Figs. 12 and 13 show the mean species distribution pattern for the whole sampling period. ${ }^{3}$ )

3) The difficult sorting out of G. fossarum and G. p. pulex was carried out completely only for part of the samples. All other times only overigerous females were identified, while the remaining animals were divided in Gammarus of Echinogammarus ( $=E$. berilloni, since no other species belonging to this genus live in the Slack). Fig. 14 shows a comparison of the distribution of Gammarus/Echinogammarus in the Slack by taking either all samples into account (solid line) or only those samples that were fully identified (broken line). Both offer the same overall picture. We may thus conclude that the fully identified samples (fig. 12) were aselectly chosen and represent the generic distribution in the Slack during our research period quite well. 


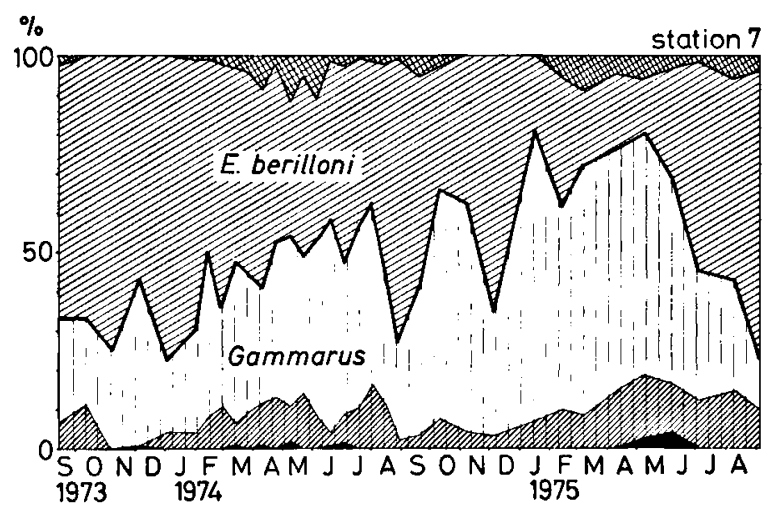

Fig. 9. Seasonal variation in the distribution of Gammarus and $E$. berilloni with the seasonal variation in the occurrence of ovigerous females of G. p. pulex (solid black), G. fossarum (chequered) and E. berilloni (chequered) at station 7. (Sample size varies from $n=73$ to $n=207$.)

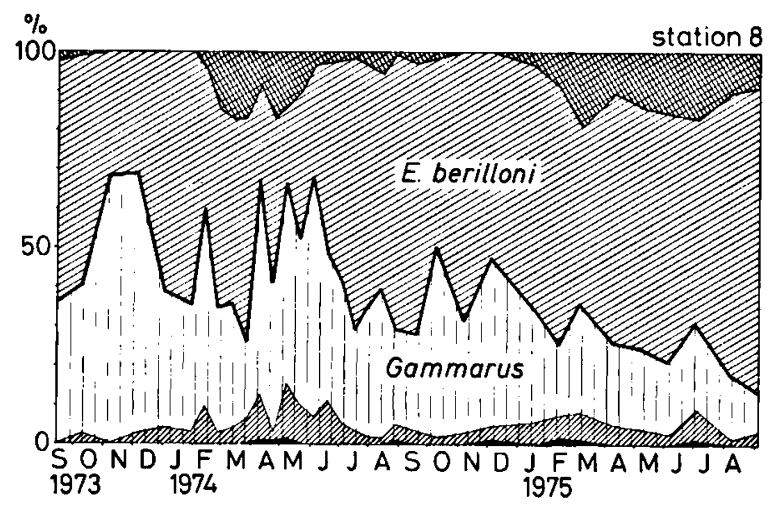

Fig. 10. Seasonal variation in the distribution of Gammarus and $E$. berilloni with the seasonal variation in the occurrence of ovigerous females of G. p. pulex (solid black), G. fossarum (chequered) and $E$. berilloni (chequered) at station 8. (Sample size varies from $n=68$ to $n=195$.)

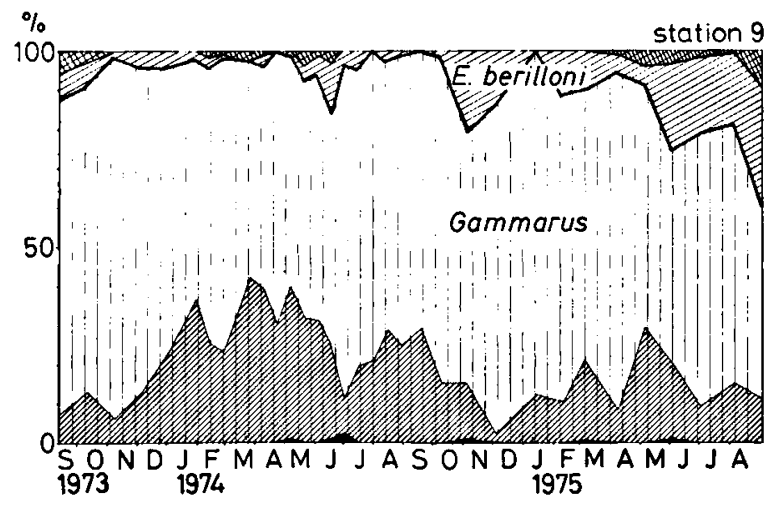

Fig. 11. Seasonal variation in the distribution of Gammarus and $E$. berilloni with the seasonal variation in the occurrence of ovigerous females of G. p. pulex (solid black), G. fossarum (chequered) and $E$. berilloni (chequered) at station 9. (Sample size varies from $n=92$ to $n=176$.)

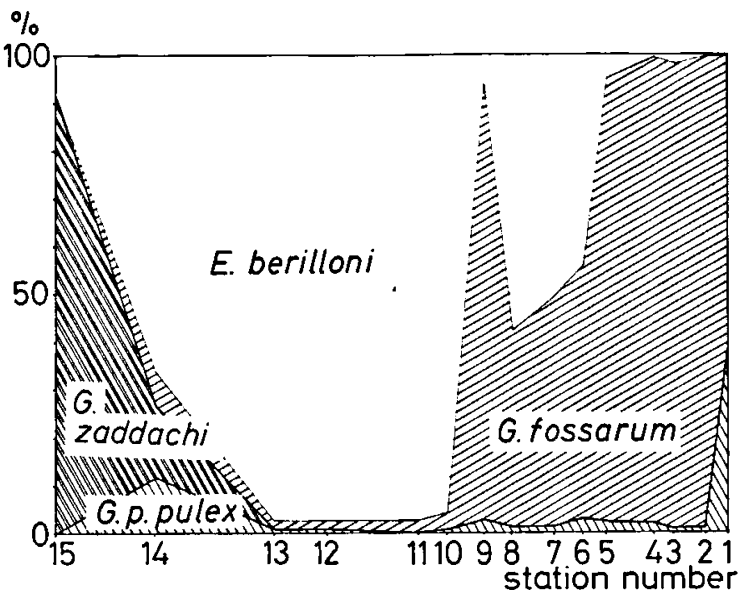

Fig. 12. Microgeographic variation in the distribution of G. p. pulex, G. fossarum, E. berilloni and G. zaddachi (species composition of only fully identified samples over entire sampling period at each station averaged).

G. p. pulex thrives best in the unstable area near the main source of the Slack (station 1). Furthermore it occurs in low percentages at most of the other stations. It lives in relatively high percentages at station 14 in the freshwater tidal area.

$G$. fossarum inhabits the upper and middle reaches of the Slack (stations 1 to 9) and occurs in small percentages at most of the other stations.

$E$. berilloni has its main distribution area in the middle and lower reaches of the Slack (stations 10 to 13 and to a somewhat lesser extent stations $6,7,8$ and 14).

G. zaddachi is a brackish-water species common at station 15; it sometimes invades the freshwater tidal area of the Slack (station 14).

The relatively high percentages of $G$. fossarum at station 9 are probably due to invasions from a nearby springbrook.

\subsubsection{Reproduction level}

The mean percentage of ovigerous females of a certain species may vary largely between different stations (fig. 15). Reproduction levels of distinct species living at the same station are sometimes quite different as well. At station 7, for instance, $G$. fossarum and $E$. berilloni occur in about the same percentages. However, the percentage of ovigerous females of $E$. berilloni is exceptionally 


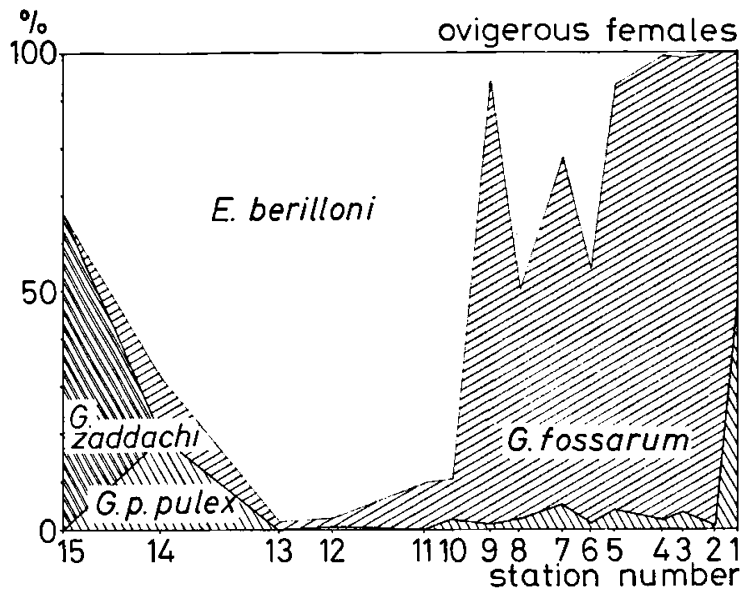

Fig. 13. Microgeographic variation in the distribution of ovigerous females of G. p. pulex, G. fossarum, E. berilloni and $G$. zaddachi (species composition of ovigerous females of only fully identified samples over entire sampling period at each station averaged).

low at this station, whereas $G$. fossarum shows a rather high reproduction level.

The high percentage of ovigerous females at some stations where a species occurs in low percentages (G. p. pulex at station 2a; G. fossarum at stations 12,13 and $14 ; E$. berilloni at stations 4 and 9) might indicate that reproducing animals colonize new areas first.

\subsubsection{Area sbift}

The distribution patterns of the gammarid species remained rather constant during the two years of sampling; only a slight shift in the percentages Gammarus/Echinogammarus was observed (fig. 16). The second sampling year (1974-1975) was warmer than the first one (1973-1974) (Goedmakers, 1980). These different weather conditions in consecutive years, and the perhaps resulting aggravated influence of pollution as well, may have caused differences in relative abundances. The second year the percentage of $G$. fossarum decreased in the populations at stations 8 and 9 (Mann-Whitney test: significance $<0.1 \%$ and $<1 \%$, respectively), while its share in the amphipod population increased at stations 6 and 7 (Mann-Whitney test: significance $<1 \%$ and $<5 \%$, respectively) (see also figs. 8 to 11 ). The combined effects of pollution and weather conditions probably have made stations 8 and 9 less

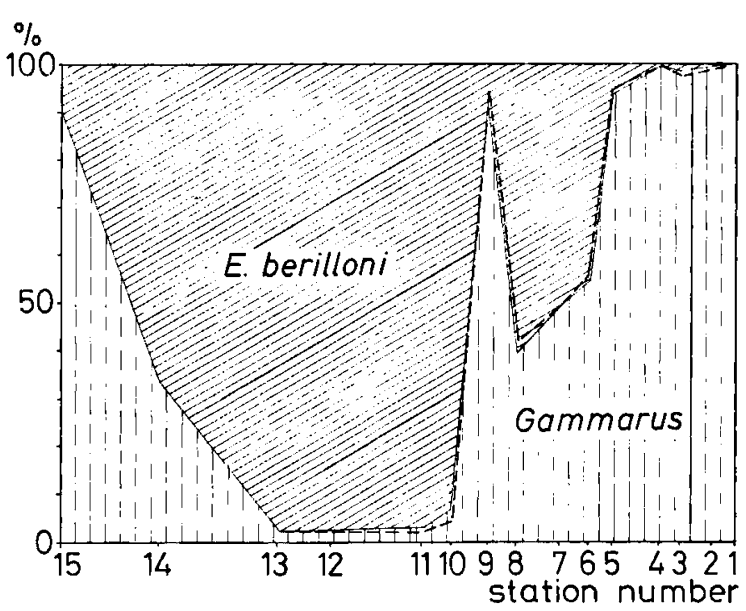

Fig. 14. Microgeographic variation in the distribution of Gammarus and E. berilloni. Comparison between the average of all samples (solid line) and the average of only those samples, that were fully identified (broken line).

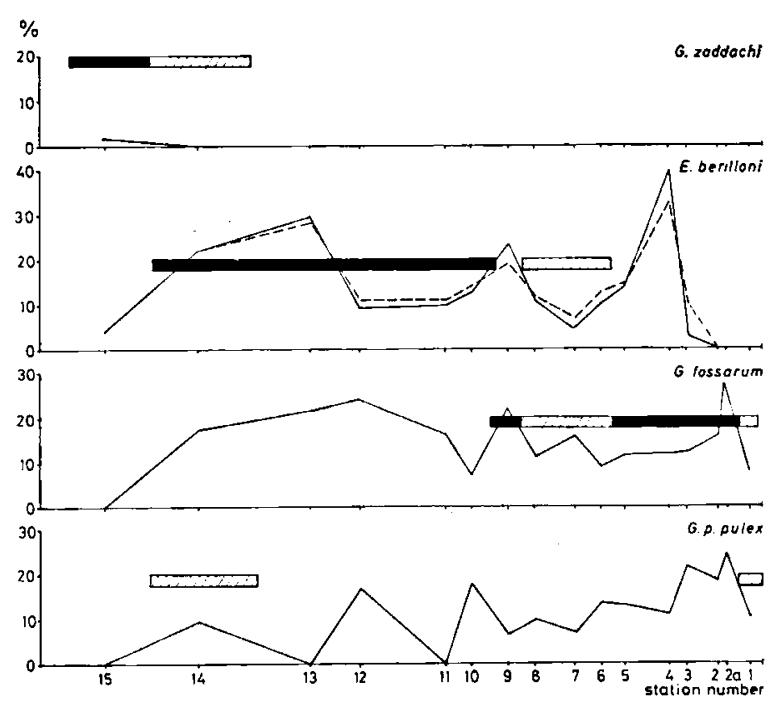

Fig. 15. Microgeographic variation in the mean percentage of ovigerous females (only fully identified samples over entire sampling period averaged) of each of the four species: G. p. pulex, G. fossarum, E. berilloni and G. zaddachi. The bars give an indication of the percentage in which each species occurs at a certain station (solid black: more than 65\%; hatched: 10-65\%). (The broken line in E. berilloni represents mean percentages of ovigerous females of this species, all samples averaged.)

favourable for $G$. fossarum or more so for $E$. berilloni in the second sampling year.

Research during summer 1978 showed that $E$. berilloni had enlarged and/or $G$. fossarum had reduced its area considerably since 1975: only station 5 was inhabited by both $E$. berilloni and $G$. fossarum in substantial percentages, whereas 


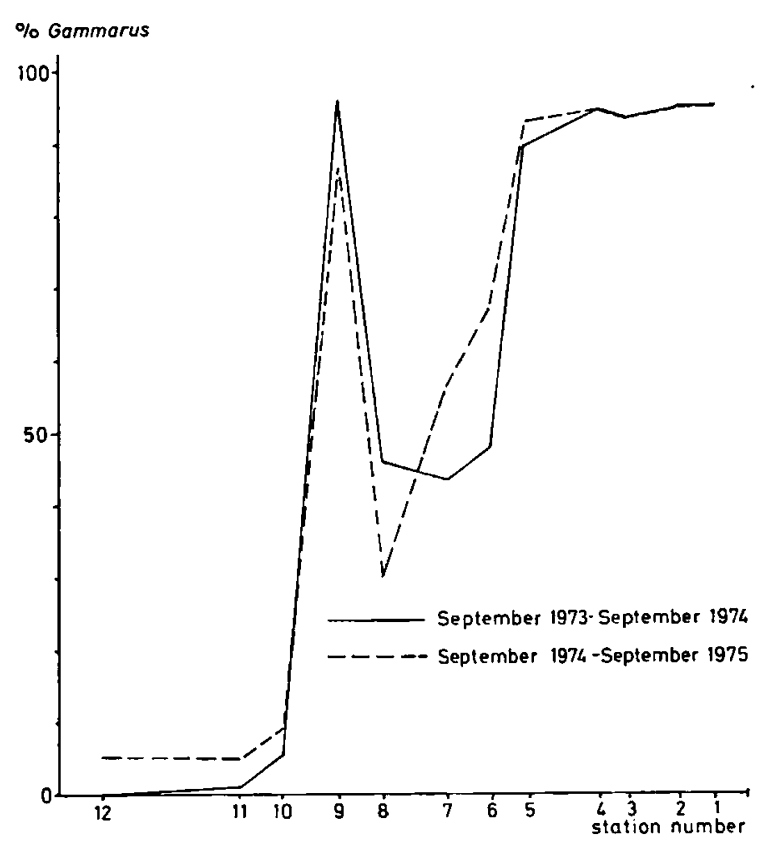

Fig. 16. Microgeographic variation in the mean percentage of Gammarus during two different years (both years the percentages of Gammarus of all samples at a certain station were averaged).

stations 6 to 9 were inhabited mainly by $E$. berilloni that year (Steenbergen et al., unpubl.).

At station 1 we found an increasing percentage of G. p. pulex in the second sampling year (fig. $5)$. This change in relative abundance of $G . p$. pulex at station 1 has not been investigated after 1975.

\subsection{Life histories}

Life cycles can be pictured by drawing histograms of the size distribution of every sample (e.g. figs. 17,18 and 19) and more simplified after computing the data the histograms contain (figs. 20 to 36). The first method gives much more detailed information on the development of a population throughout the year, but makes it difficult to compare different stations at first sight.

Figs. 20 to 36 show the seasonal variation in mean cephalic length, its standard deviation and the percentage of ovigerous females of a species or genus at a certain station. The standard deviation of the mean cephalic length is an indication for the sudden in- or decrease of some size categories: the hatching of juveniles, the migration of large animals or the death of old animals may account for a change in standard deviation of mean cephalic length. Although this is a very gross method to compress our data (most samples show no normal distribution), it was the most useful way, considering the enormous mass of data we collected.

\section{G. p. pulex (figs. 17 and 20)}

It was impossible to investigate the life cycle of $G$. p. pulex as thoroughly as that of $G$. fossarum (see below), because only one large and rather thriving population exists in the Slack at station 1. Elsewhere in this river the species is found only sporadically. Therefore we decided not to delve deeply into the life history of G. p. pulex.

We conclude about the life history of $G . p$. pulex, that distinct peaks in its sexual activity occur during the year. Consequently, the production of offspring is periodic. Reproduction peak times were in different months during both observation years (January and July 1974; May 1975).

Although the interpretation of the data illustrated in fig. 17 is a little difficult, since they are based on comparatively few animals and show large between-year differences, we feel justified to draw some conclusions about the life cycle of G.p.pulex at station 1. The comparison of the length of ovigerous females with that of other size categories, and the percentage of the total G. p. pulex population each size category formed (fig. 17), were of particular importance to our conclusions: G. $p$. pulex lives about one year; most females produce offspring once or twice, only in rare cases three times, a year.

\section{G. fossarum (figs. 18 and 21 to 30 )}

The life cycles of $G$. fossarum 4 ) at different stations and in different years reveal important distinctions. The species can have a very low (station 1, fig. 21) or a very high (stations $2 \mathrm{a}$ and 9, figs. 23 and 30 ) level of sexual activity. At most stations reproduction shows a certain periodicity

4) We identified most samples only down to the genus. Fig. 37 shows that our results on Gammarus might be considered as results for $G$. fossarum, due to the very low percentages of G. p. pulex living at stations 2 to 9 (see also section 5.2.2 and fig. 14). 

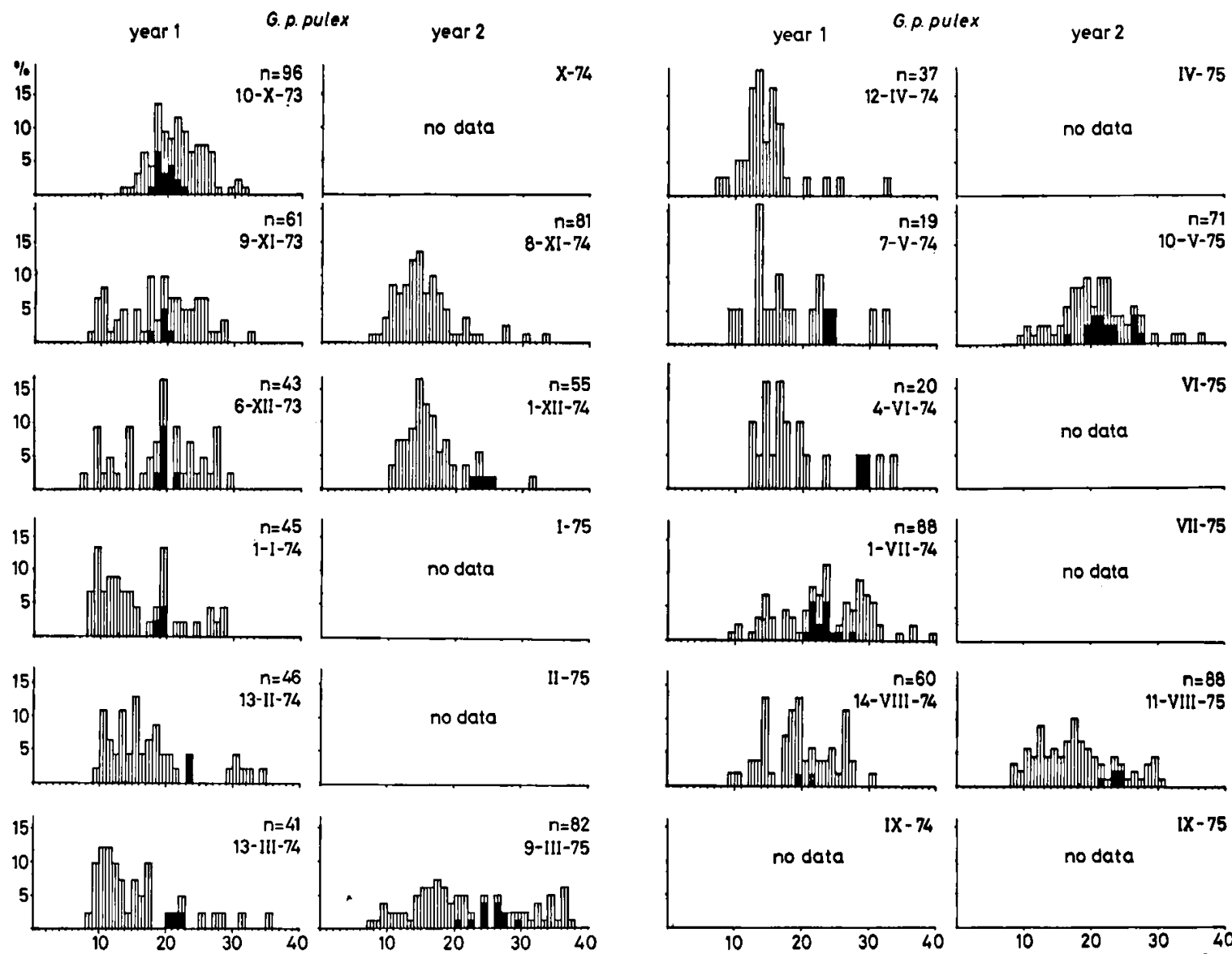

Fig. 17. Size frequency during two years of G. p. pulex at station 1. Black parts represent ovigerous females. Cephalic lengths are expressed in micrometer units ( 1 unit $=0.05 \mathrm{~mm}$ ).

with minima in late autumn. Sometimes, this periodicity is very clear, as in stations $2 a$ or 6 (figs. 23 and 27 ), or it can be almost absent, as in station 2 (fig. 22).

There is a wide range of between-year variations. At station 3 (fig. 24) sexual activity was much higher during 1975 than in 1974, while at station 9 (fig. 30) this was the other way round. Also the time of the year in which sexual activity is highest differs along the river between various years. At station 5 (fig. 26) a maximum in sexual activity occurred the first year in May, the second year in February. In general, gammarids in the Slack are sexually active in spring, while sexual activity was as high or higher during the second sampling year.

Most offspring is produced in summer, but since ovigerous females are found almost at all times, so are juveniles. Overlapping life cycles make the results difficult to interpret, but a production of two batches of juveniles in a maximal life period of about twelve months seems most common.

As in $E$. berilloni (see below) we found two strains of animals, a short and a long living one. A peak of juveniles appears in the beginning of the year. These animals become ovigerous in spring and produce offspring in summer. This second peak of juveniles becomes ovigerous in autumn for the first time and again in spring. Both strains of animals disappear from our samples in summer.

E. berilloni (figs. 19 and 31 to 36 )

Life cycles of $E$. berilloni appeared to be roughly similar at different localities. We observed, how- 

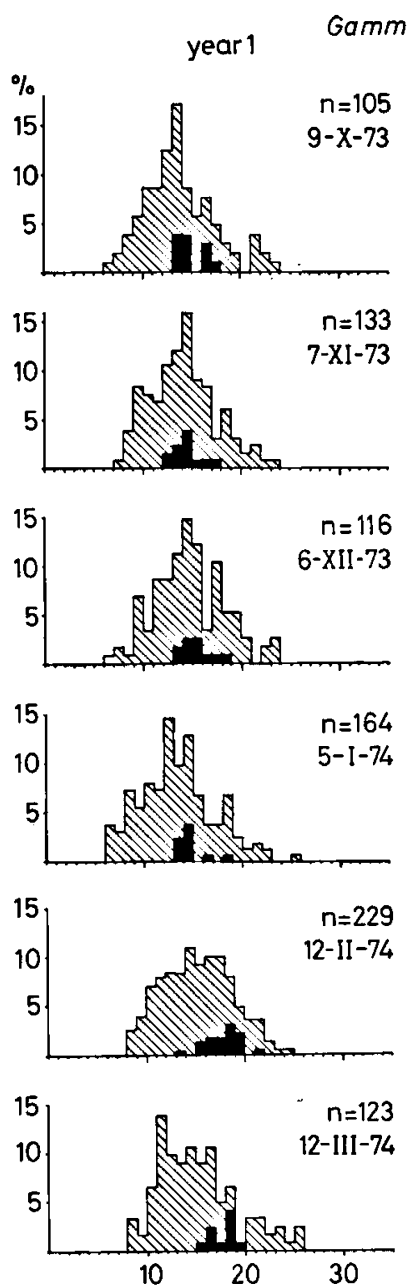

year 2
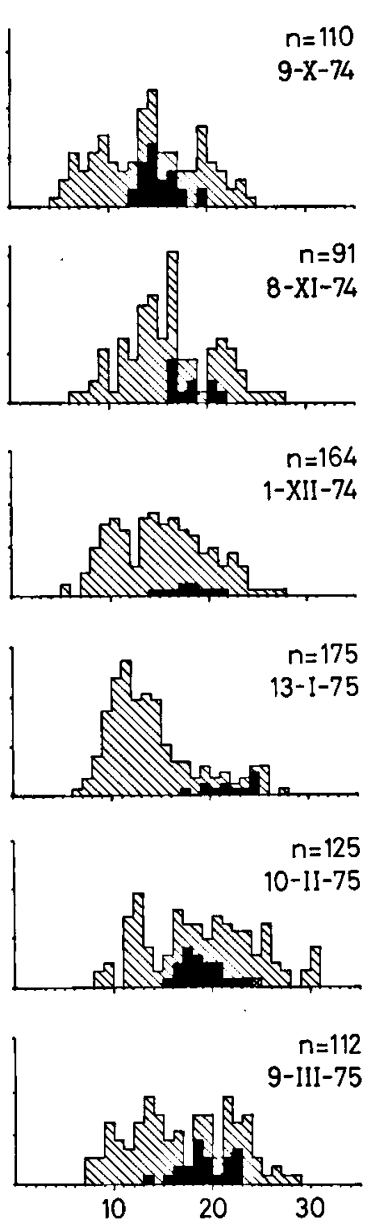

year 1 Gammarus

year 2
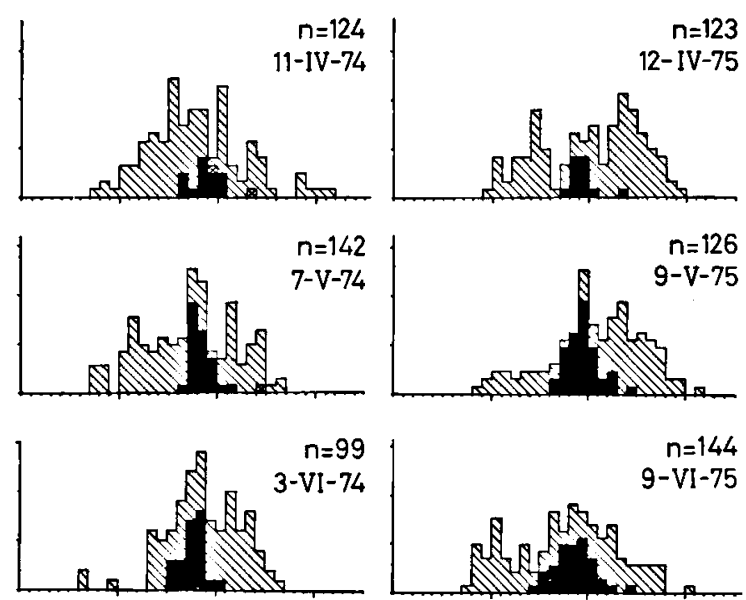

$n=144$

$9-V I-75$
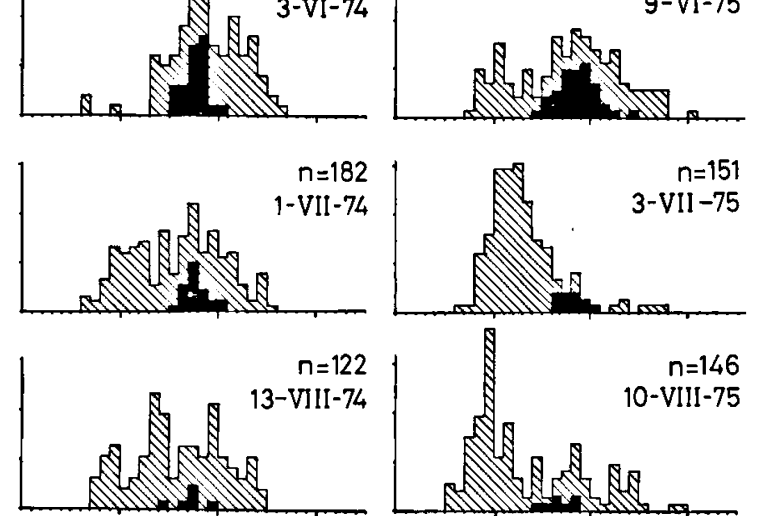

$n=146$
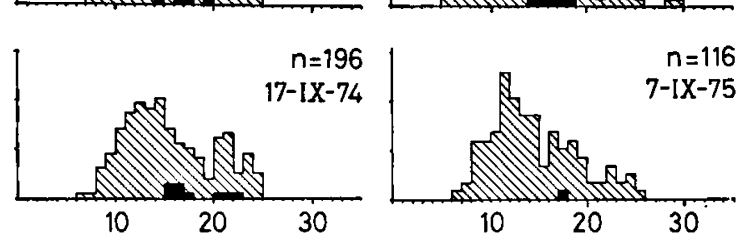

Fig. 18. Size frequency during two years of Gammarus at station 4. Black parts represent ovigerous females of $G$. fossarum, a cross represents an ovigerous $G$. p. pulex. Cephalic lengths are expressed in micrometer units $(1 \mathrm{unit}=0.05 \mathrm{~mm})$.

ever, a pronounced difference between the life histories in two consecutive years of investigation.

A rather long period with no sexual activity (autumn/winter 1973) was followed by a long (spring 1974) and a short (September/October 1974) period of high sexual activity. The next year showed a much shorter inactive period (November/December 1974) followed by a very long period of high sexual activity, lasting all spring and summer 1975 (figs. 31 to 36 ).

The detailed information of fig. 19 clearly shows (compare percentages and relative positions of size categories with ovigerous females) that most females produce offspring once, while few hatch two batches of juveniles during their maximal twelve-month lifespan.

Juveniles appear in our samples in November. These animals become ovigerous in the beginning of the next year, produce offspring in June and disappear in summer. This offspring becomes ovigerous in autumn. These ovigerous females produce the first mentioned juveniles of November and may live to produce a second batch of offspring at the same time as their own offspring reproduces. They disappear also in summer from our samples.

Concluding, we found two strains of animals, the first living about nine months and producing 

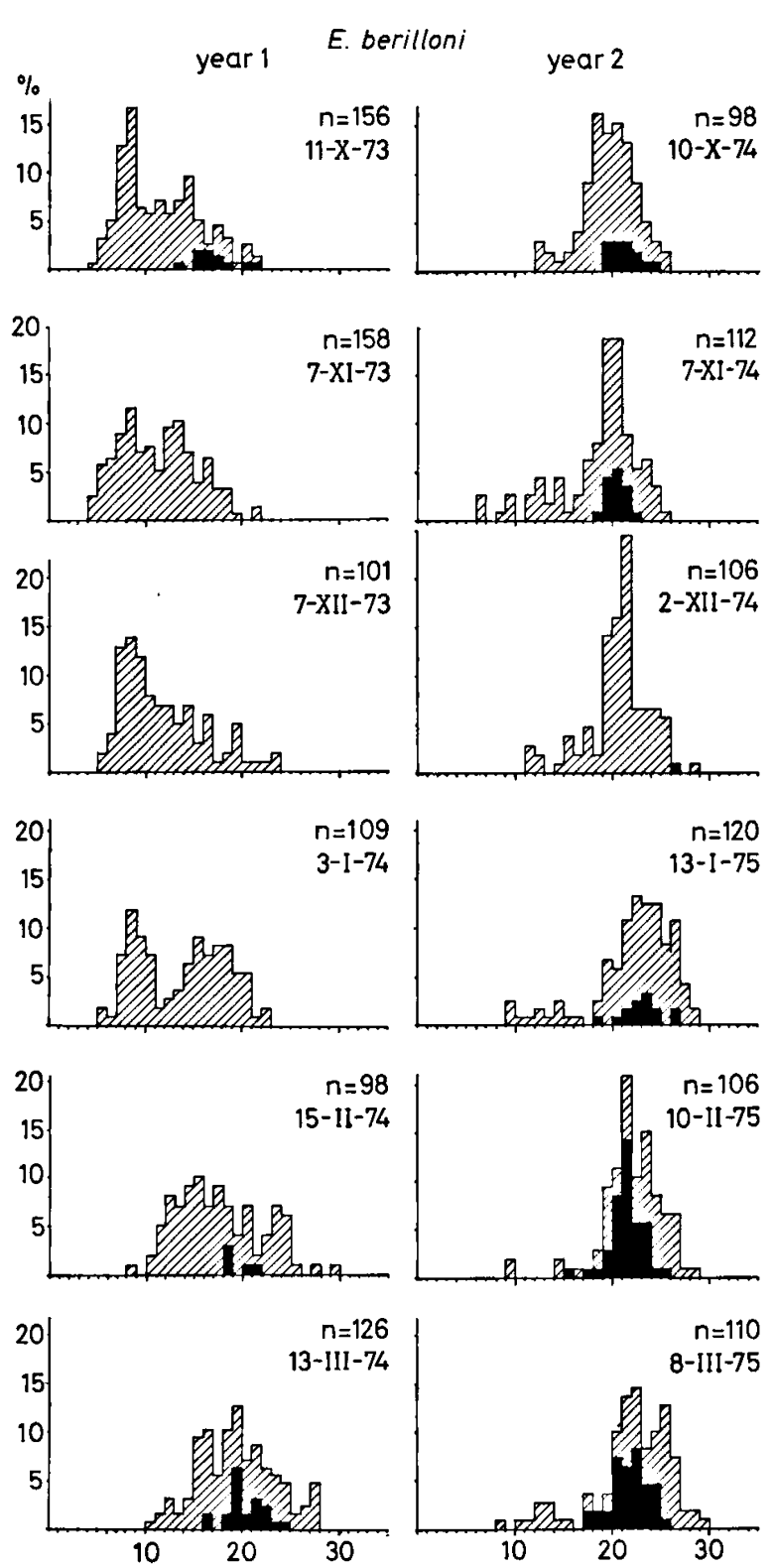

Fig. 19. Size frequency during two years of E. berilloni at station 10. Black parts represent ovigerous females. Cephalic lengths are expressed in micrometer units ( 1 unit $=0.05 \mathrm{~mm}$ ).

one batch of offspring, the second one living up to one year and producing one or two batches of offspring.

\subsubsection{Growth and size}

Different species grow at a different pace even under the same environmental conditions (compare mean cephalic lengths of Gammarus and Echinogammarus at the same stations in tables IV
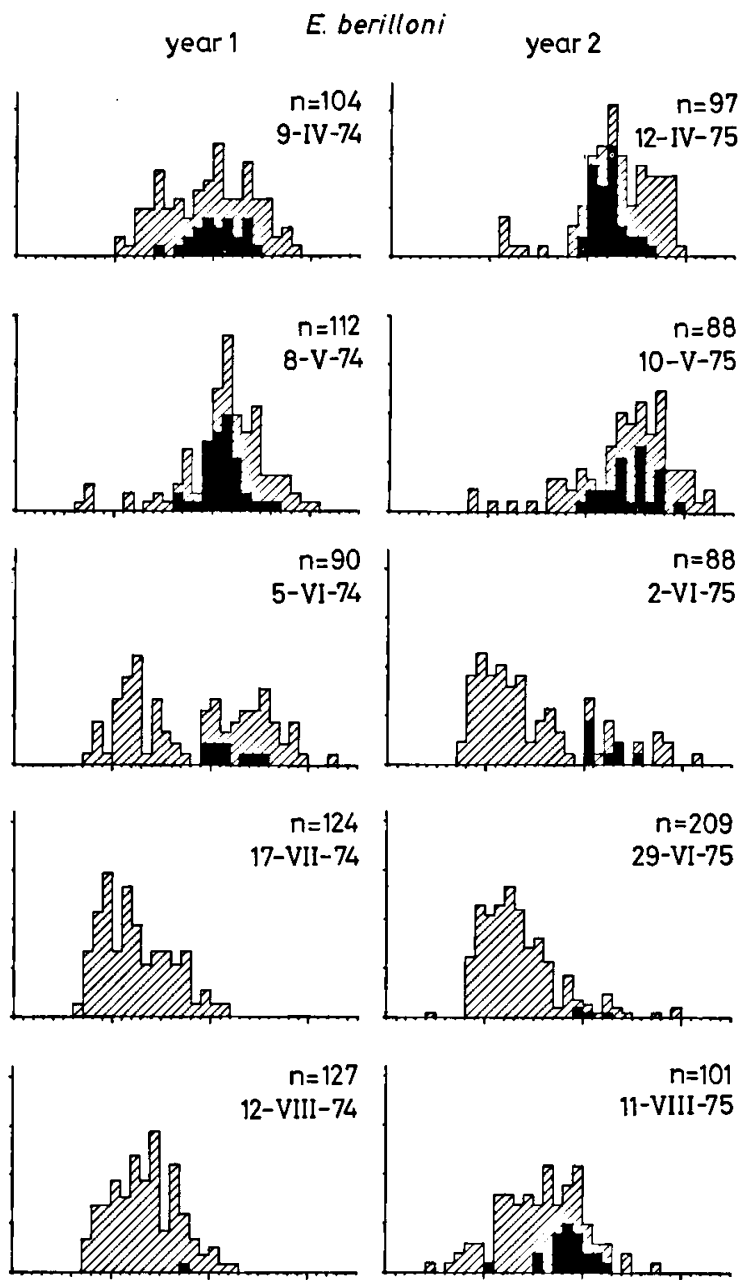

$n=101$ $11-$ VIII-75

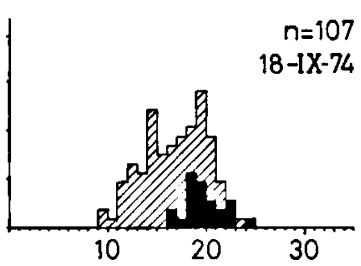

$n=122$

7-IX-75

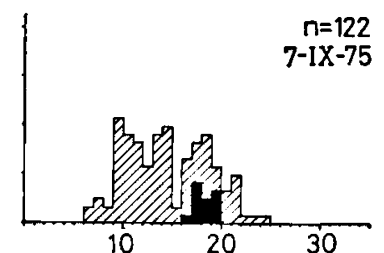

and V). Under different environmental conditions the growth rates of animals of the same species may be very dissimilar (compare different stations and different years within table IV or V).

As gammarids grow, their cephalic length increases. Thus, the mean cephalic length of a population increases when all animals mature. Inversely, the appearance of juveniles causes a decrease in mean cephalic length, as does the death of old 
-mean cephalic length --.-SD cephalic length \#percentage ovigerous females

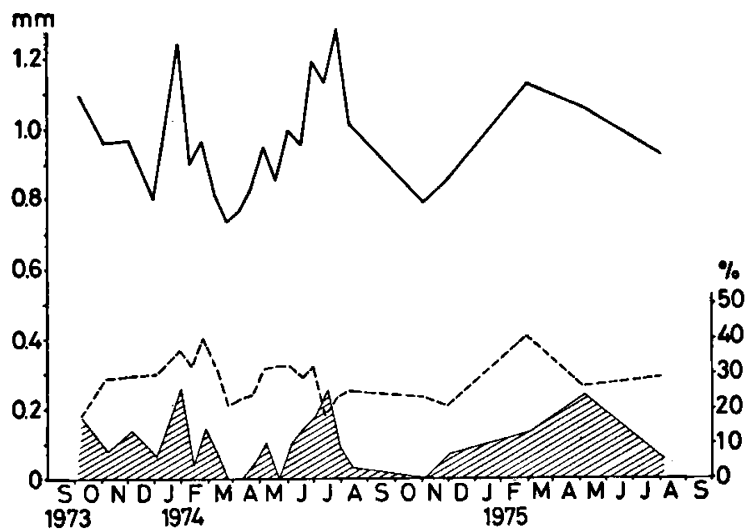

Fig. 20. Seasonal variation in sexual activity (\% ovigerous females), mean size and its standard deviation of $G$. $p$. pulex at station 1. (Sample size varies between $n=10$ and $n=96)$.

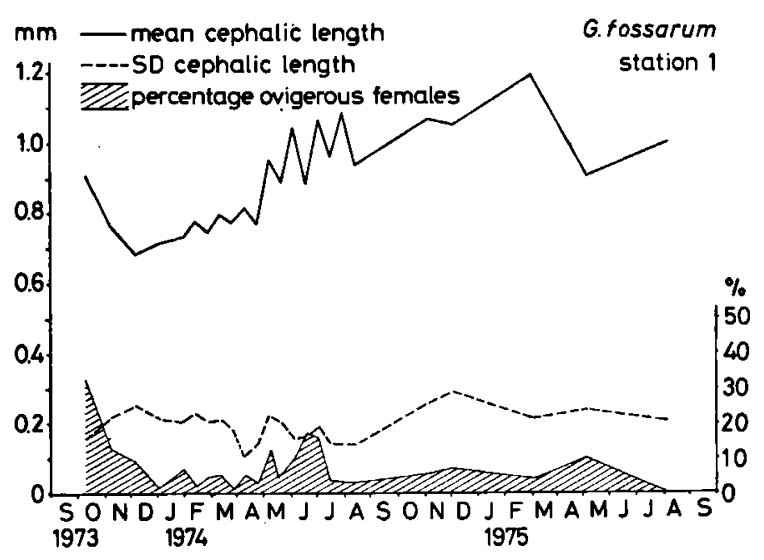

Fig. 21. Seasonal variation in sexual activity (\% ovigerous females), mean size and its standard deviation of $G$. fossarum at station 1. (Sample size varies between $n=24$ and $n=167$. .)

animals. A rather complicated picture is the result, depending on the percentage of each developmental stage present in a population. Variations in the standard deviation of the mean cephalic length point to changes in population structure.

\subsubsection{Interspecific differences.}

The three species show important differences in growth rates and maximum sizes. G. p. pulex is the largest species, while $G$. fossarum is the smallest (figs. 17, 18 and 19; tables IV and V).

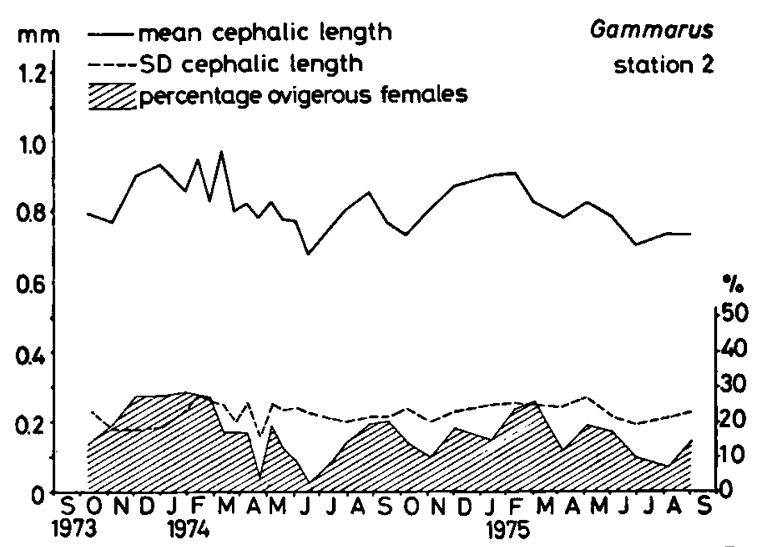

Fig. 22. Seasonal variation in sexual activity (\% ovigerous females), mean size and its standard deviation of Gammarus at station 2. (Sample size varies between $n=82$ and $n=179$.

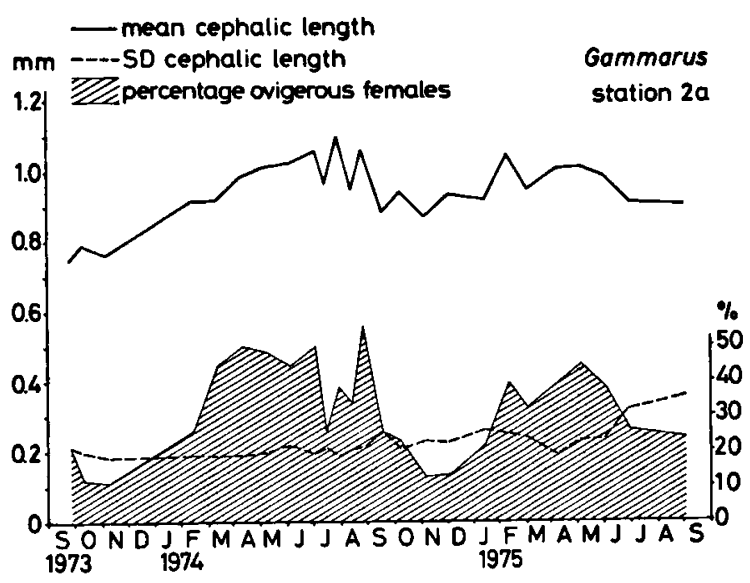

Fig. 23. Seasonal variation in sexual activity (\% ovigerous females), mean size and its standard deviation of Gammarus at station 2a. (Sample size varies between $n=99$ and $n=191$.)

\subsubsection{Microgeographic variation.}

Also within each species, animals attain different maximum and mean lengths, and have different growth rates at different stations. There are clear differences in mean size between the Gammarus populations of different stations (fig. 38). Compare for instance the mean lengths of the Gammarus populations at stations 2 and 2 a during our sampling period (figs. 22 and 23 ). The mean size of gammarids at station $2 \mathrm{a}$ is nearly always much larger than of those living at station 2 , which is situated within a distance of a few metres only. 


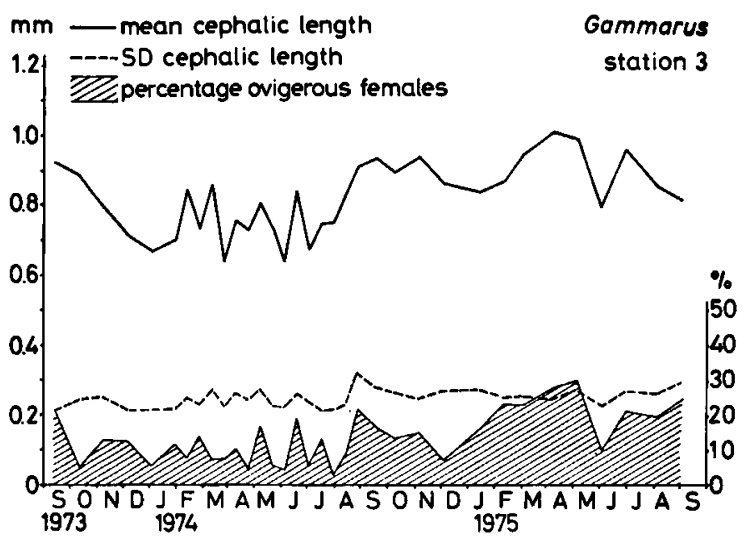

Fig. 24. Seasonal variation in sexual activity (\% ovigerous females), mean size and its standard deviation of Gammarus at station 3. (Sample size varies between $n=70$ and $n=286$.

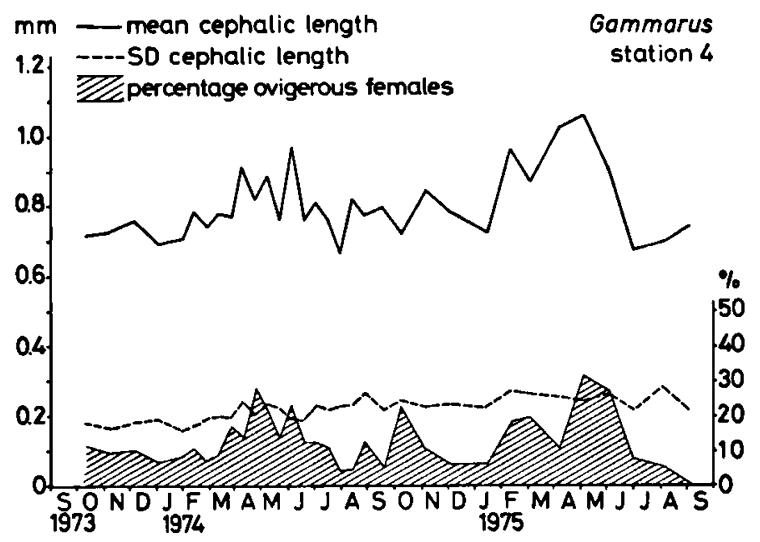

Fig. 25. Seasonal variation in sexual activity (\% ovigerous females), mean size and its standard deviation of Gammarus at station 4. (Sample size varies between $n=90$ and $n=290$.)

A complicating factor in the explanation of data like these, may be the presence of more animals in an advanced life stage at certain stations, resulting in a larger mean length. This could mean that animals grow faster at station $2 a$, or that adult animals prefer station $2 a$ while younger animals are found at station 2. A comparison however, of data on sexual activity and mean cephalic lengths (table IV) demonstrates that differences in maturity of a population can not provide the sole explanation for the observed differences in cephalic length. For example, at station $2 \mathrm{a}$ the mean cephalic length of the total population is slightly smaller in year 1 than in year 2, while

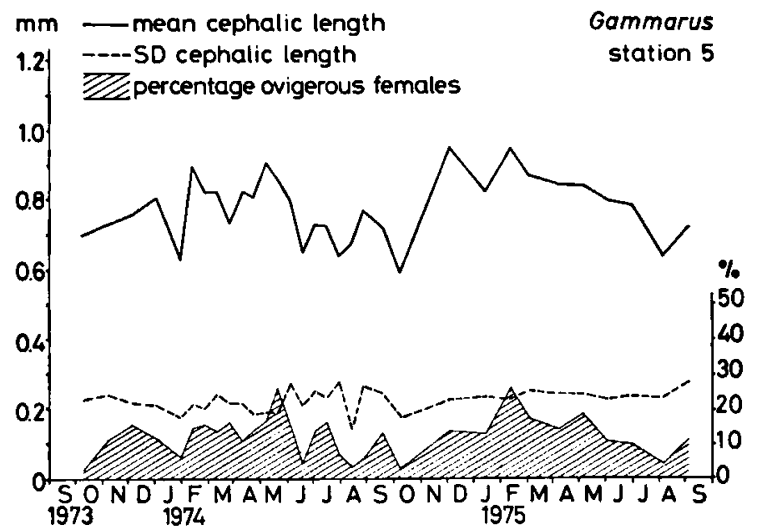

Fig. 26. Seasonal variation in sexual activity (\% ovigerous females), mean size and its standard deviation of Gammarus at station 5. (Sample size varies between $n=87$ and $n=220$.)

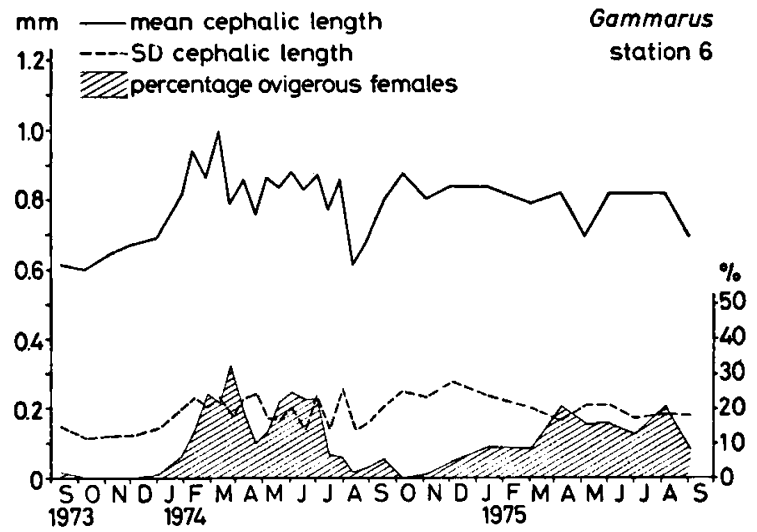

Fig. 27. Seasonal variation in sexual activity (\% ovigerous females), mean size and its standard deviation of Gammarus at station 6. (Sample size varies between $n=29$ and $n=220$.

the percentage of ovigerous females is larger in the first year.

The same microgeographic variation in size can be observed while comparing the mean cephalic lengths of ovigerous females at different stations (table IV and fig. 38). Even animals in the same life stage, in this case ovigerous females, show a considerable variation in mean cephalic lengths between the various stations, as was the case for the total populations. Another explanation, besides that of a difference in maturity between various populations, might be that at some stations animals reach maturity after fewer moults. This could explain the smaller mean size of such a population 


\section{TABLE IV}

Summary of data on mean size and sexual activity of Gammarus at the different sampling stations in the Slack over the entire sampling period. This table is partly based on data of Teixeira de Mattos (unpubl.).

total population number of animals and percentage ovigerous mean cephalic length $(\bar{x})$ in $\mathrm{mm}$ females ovigerous females

number of animals and mean cephalic length $(\bar{x})$ in $\mathrm{mm}$
G. fossarum
G. p. pulex

\begin{tabular}{|c|c|c|c|c|c|c|c|c|c|c|c|c|c|c|}
\hline & & & & \\
\hline & \multicolumn{2}{|c|}{ year 1} & \multicolumn{2}{|c|}{ year 2} & \multirow{2}{*}{$\begin{array}{c}\text { year } 1 \\
\%\end{array}$} & \multirow{2}{*}{$\begin{array}{c}\text { year } 2 \\
\%\end{array}$} & \multicolumn{2}{|c|}{ year 1} & \multicolumn{2}{|c|}{ year 2} & \multicolumn{2}{|c|}{ year 1} & \multicolumn{2}{|c|}{ year 2} \\
\hline & $n$ & $\bar{x}$ & $n$ & $\bar{x}$ & & & $n$ & $\bar{x}$ & $n$ & $\bar{x}$ & $n$ & $\bar{x}$ & $n$ & $\bar{x}$ \\
\hline Sta. 1-15 & 20703 & 0.83 & 15210 & 0.87 & 15.2 & 16.2 & 2960 & 0.91 & 2298 & 0.96 & 163 & 1.09 & 167 & 1.20 \\
\hline Sta. 1 & 2540 & 0.93 & 1671 & 0.98 & 10.0 & 9.9 & 163 & 0.92 & 74 & 1.03 & 91 & 1.10 & 91 & 1.23 \\
\hline Sta. 2 & 2161 & 0.83 & 1636 & 0.80 & 17.0 & 15.9 & 367 & 0.89 & 255 & 0.94 & 2 & & 5 & \\
\hline Sta. $2 \mathrm{a}$ & 1596 & 0.94 & 1447 & 0.95 & 34.1 & 29.6 & 534 & 0.94 & 418 & 0.96 & 11 & & 11 & \\
\hline Sta. 3 & 2651 & 0.78 & 1616 & 0.89 & 10.7 & 17.9 & 275 & 0.87 & 278 & 0.96 & 9 & & 11 & \\
\hline Sta. 4 & 2808 & 0.77 & 1604 & 0.83 & 11.7 & 13.3 & 321 & 0.87 & 211 & 0.94 & 8 & & 2 & \\
\hline Sta. 5 & 2583 & 0.75 & 1672 & 0.78 & 11.2 & 12.1 & 281 & 0.90 & 192 & 0.95 & 9 & & 10 & \\
\hline Sta. 6 & 1256 & 0.75 & 1150 & 0.79 & 10.0 & 12.1 & 111 & 0.93 & 135 & 0.93 & 6 & & 4 & \\
\hline Sta. 7 & 1161 & 0.80 & 900 & 0.89 & 19.9 & 17.6 & 211 & 0.93 & 150 & 0.95 & 8 & & 8 & \\
\hline Sta. 8 & 1076 & 0.86 & 444 & 0.88 & 12.3 & 15.3 & 127 & 0.91 & 62 & 0.97 & 5 & & 6 & \\
\hline Sta. 9 & 2079 & 0.93 & 1349 & 0.92 & 26.7 & 18.1 & 549 & 0.94 & 241 & 0.98 & 5 & & 3 & \\
\hline Sta. 10 & 147 & 0.75 & 154 & 0.82 & 10.9 & 8.4 & 12 & 0.93 & 11 & 0.92 & 4 & & 2 & \\
\hline Sta. 11 & 20 & 0.62 & 73 & 0.85 & 10.0 & 17.8 & 2 & 0.88 & 13 & 0.93 & 0 & & 0 & \\
\hline Sta. 12 & 9 & 0.57 & 70 & 0.91 & 11.1 & 18.6 & 1 & 0.95 & 9 & 1.00 & 0 & & 4 & \\
\hline Sta. 13 & 19 & 0.79 & 3 & 0.94 & 5.3 & 66.7 & 1 & 1.00 & 2 & 0.94 & 0 & & 0 & \\
\hline Sta. 14 & 51 & 1.20 & 11 & 0.80 & 11.8 & 18.2 & 1 & 0.90 & 2 & 0.90 & 5 & & 0 & \\
\hline Sta. 15 & 518 & 0.90 & 3 & 1.12 & 2.1 & 0.0 & 0 & & 0 & & 0 & & 0 & \\
\hline Sta. 2-15 & & & & & & & & & & & 72 & 1.09 & 76 & 1.16 \\
\hline
\end{tabular}

\begin{tabular}{|c|c|c|c|c|c|c|c|c|c|c|c|c|c|c|}
\hline & & & & \\
\hline & \multicolumn{2}{|c|}{ year 1} & \multicolumn{2}{|c|}{ year 2} & \multirow{2}{*}{$\begin{array}{c}\text { year } 1 \\
\%\end{array}$} & \multirow{2}{*}{$\begin{array}{c}\text { year } 2 \\
\%\end{array}$} & \multicolumn{2}{|c|}{ year 1} & \multicolumn{2}{|c|}{ year 2} & \multicolumn{2}{|c|}{ year 1} & \multicolumn{2}{|c|}{ year 2} \\
\hline & $n$ & $\bar{x}$ & $n$ & $\bar{x}$ & & & $n$ & $\bar{x}$ & $n$ & $\bar{x}$ & $n$ & $\bar{x}$ & $n$ & $\bar{x}$ \\
\hline Sta. 1-15 & 20703 & 0.83 & 15210 & 0.87 & 15.2 & 16.2 & 2960 & 0.91 & 2298 & 0.96 & 163 & 1.09 & 167 & 1.20 \\
\hline Sta. 1 & 2540 & 0.93 & 1671 & 0.98 & 10.0 & 9.9 & 163 & 0.92 & 74 & 1.03 & 91 & 1.10 & 91 & 1.23 \\
\hline Sta. 2 & 2161 & 0.83 & 1636 & 0.80 & 17.0 & 15.9 & 367 & 0.89 & 255 & 0.94 & 2 & & 5 & \\
\hline Sta. $2 \mathrm{a}$ & 1596 & 0.94 & 1447 & 0.95 & 34.1 & 29.6 & 534 & 0.94 & 418 & 0.96 & 11 & & 11 & \\
\hline Sta. 3 & 2651 & 0.78 & 1616 & 0.89 & 10.7 & 17.9 & 275 & 0.87 & 278 & 0.96 & 9 & & 11 & \\
\hline Sta. 4 & 2808 & 0.77 & 1604 & 0.83 & 11.7 & 13.3 & 321 & 0.87 & 211 & 0.94 & 8 & & 2 & \\
\hline Sta. 5 & 2583 & 0.75 & 1672 & 0.78 & 11.2 & 12.1 & 281 & 0.90 & 192 & 0.95 & 9 & & 10 & \\
\hline Sta. 6 & 1256 & 0.75 & 1150 & 0.79 & 10.0 & 12.1 & 111 & 0.93 & 135 & 0.93 & 6 & & 4 & \\
\hline Sta. 7 & 1161 & 0.80 & 900 & 0.89 & 19.9 & 17.6 & 211 & 0.93 & 150 & 0.95 & 8 & & 8 & \\
\hline Sta. 8 & 1076 & 0.86 & 444 & 0.88 & 12.3 & 15.3 & 127 & 0.91 & 62 & 0.97 & 5 & & 6 & \\
\hline Sta. 9 & 2079 & 0.93 & 1349 & 0.92 & 26.7 & 18.1 & 549 & 0.94 & 241 & 0.98 & 5 & & 3 & \\
\hline Sta. 10 & 147 & 0.75 & 154 & 0.82 & 10.9 & 8.4 & 12 & 0.93 & 11 & 0.92 & 4 & & 2 & \\
\hline Sta. 11 & 20 & 0.62 & 73 & 0.85 & 10.0 & 17.8 & 2 & 0.88 & 13 & 0.93 & 0 & & 0 & \\
\hline Sta. 12 & 9 & 0.57 & 70 & 0.91 & 11.1 & 18.6 & 1 & 0.95 & 9 & 1.00 & 0 & & 4 & \\
\hline Sta. 13 & 19 & 0.79 & 3 & 0.94 & 5.3 & 66.7 & 1 & 1.00 & 2 & 0.94 & 0 & & 0 & \\
\hline Sta. 14 & 51 & 1.20 & 11 & 0.80 & 11.8 & 18.2 & 1 & 0.90 & 2 & 0.90 & 5 & & 0 & \\
\hline Sta. 15 & 518 & 0.90 & 3 & 1.12 & 2.1 & 0.0 & 0 & & 0 & & 0 & & 0 & \\
\hline Sta. 2-15 & & & & & & & & & & & 72 & 1.09 & 76 & 1.16 \\
\hline
\end{tabular}

In the first and the last lines data on stations $4 \mathrm{a}$ and $4 \mathrm{~b}$ are included (cf. Goedmakers, 1980).

Year 1 summarizes data of the sampling period September 1973 to September 1974; year 2 those of September 1974 to September 1975.

Since this table contains data on all animals belonging to the genus Gammarus, data for G. zaddachi are included in the results of stations 14 and 15.

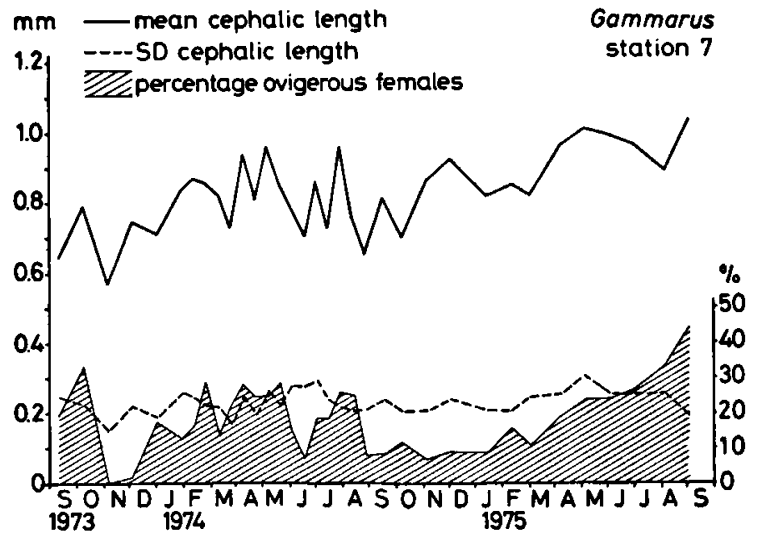

Fig. 28. Seasonal variation in sexual activity (\% ovigerous females), mean size and its standard deviation of Gammarus at station 7. (Sample size varies between $n=23$ and $n=95$.

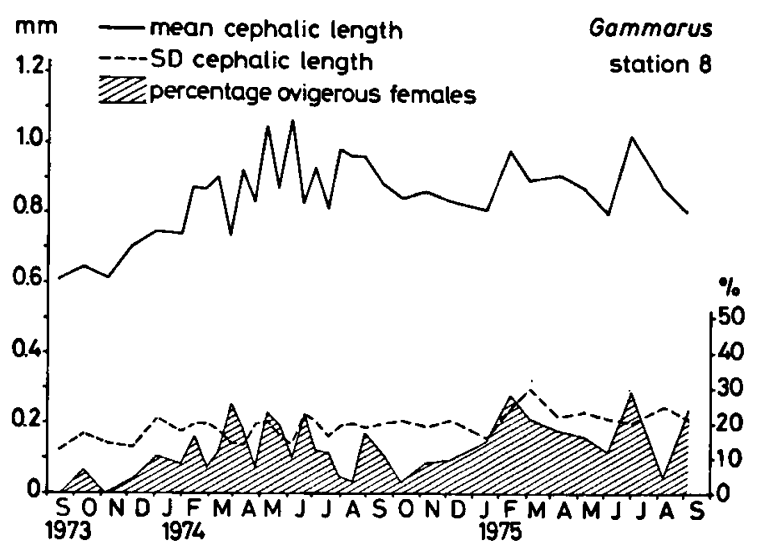

Fig. 29. Seasonal variation in sexual activity (\% ovigerous females), mean size and its standard deviation of Gammarus at station 8. (Sample size varies between $n=17$ and $n=100$.) 


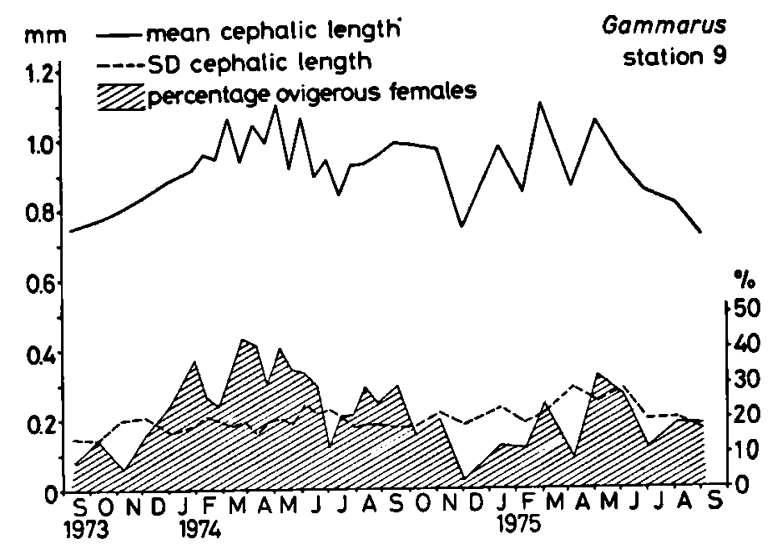

Fig. 30. Seasonal variation in sexual activity (\% ovigerous females), mean size and its standard deviation of Gammarus at station 9. (Sample size varies between $n=77$ and $n=176$.

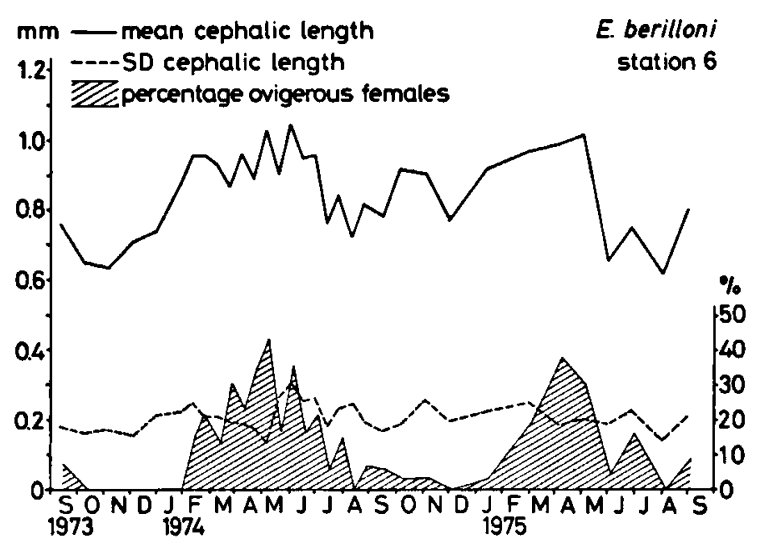

Fig. 31. Seasonal variation in sexual activity (\% ovigerous females), mean size and its standard deviation of $E$. berilloni at station 6. (Sample size varies between $n=10$ and $n=111$.

as well as the smaller size of ovigerous females. Both growth rates and the number of moults necessary to reach maturity would have to be investigated more thoroughly before one could decide which of either processes causes these population differences.

A preference of certain size groups for particular localities seems another obvious explanation of part of this problem. Much research has been done on e.g. the size distribution of gammarids over different types of substrate at a certain locality (Roux, 1971; Rees, 1972 and Miller \& Buikema, 1977). However, totally different population

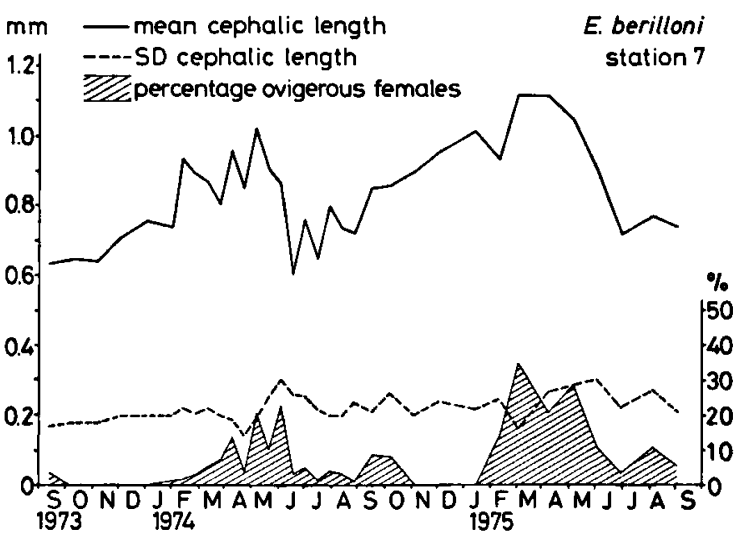

Fig. 32. Seasonal variation in sexual activity (\% ovigerous females), mean size and its standard deviation of $E$. berilloni at station 7. (Sample size varies between $n=22$ and $n=158$.)

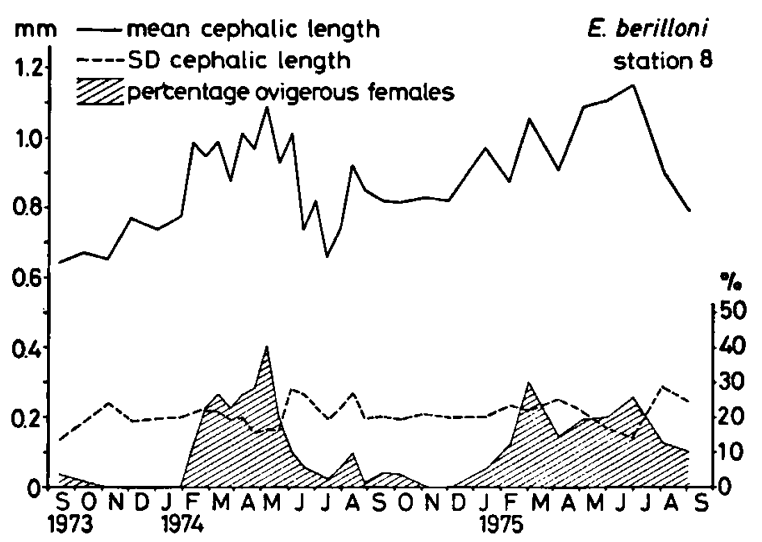

Fig. 33. Seasonal variation in sexual activity (\% ovigerous females), mean size and its standard deviation of $E$. berilloni at station 8. (Sample size varies between $n=30$ and $n=145$.)

structures at two localities would point towards a migration of certain size groups from one locality to another. For station 2 the large animals would have to migrate to station $2 \mathrm{a}$ or the small animals from station $2 \mathrm{a}$ towards 2 . At station 2 however, hardly any upstream migration activity was found, while the drifting animals at station $2 a$ were definitely not the smallest ones (Goedmakers \& Pinkster, in press).

Although differences in cephalic length between different stations are more obvious in $G$. fossarum, they can also be observed in E. berilloni (table V and fig. 39). In both species the most likely 


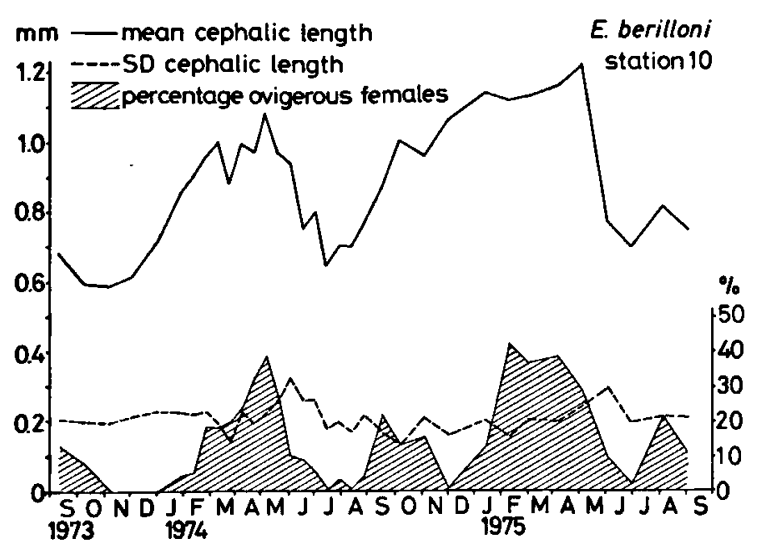

Fig. 34. Seasonal variation in sexual activity (\% ovigerous females), mean size and its standard deviation of $E$. berilloni at station 10. (Sample size varies between $n=88$ and $n=209$.)

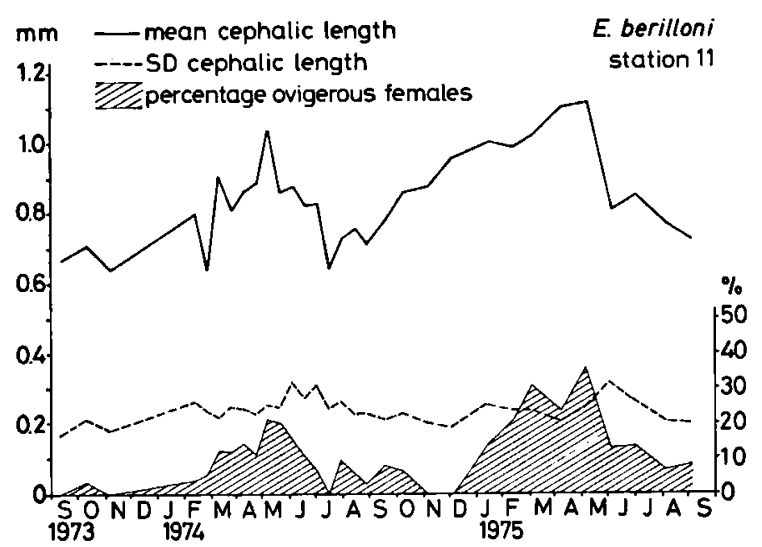

Fig. 35. Seasonal variation in sexual activity (\% ovigerous females), mean size and its standard deviation of $E$. berilloni at station 11. (Sample size varies between $n=93$ and $n=193$.)

explanation for these differences in size seems to be the combined influence of different growth rates and different population structures at different stations.

\subsubsection{Seasonal and annual variation.}

The seasonal fluctation in mean cephalic length is very clear in $E$. berilloni (figs. 31 to 36 ), due to its periodical reproduction. Since $G$. fossarum reproduces at most stations throughout the year, seasonal fluctuation in mean cephalic length of Gammarus populations (figs. 21 to 30 ) is less

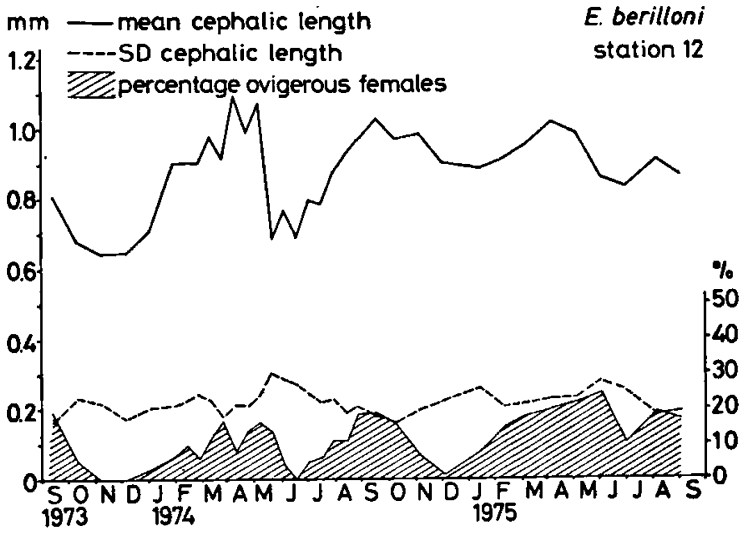

Fig. 36. Seasonal variation in sexual activity (\% ovigerous females), mean size and its standard deviation of $E$. berilloni at station 12. (Sample size varies between $n=86$ and n. $=$ 169.)

marked. We observed a periodicity in mean cephalic length for G. p. pulex (fig. 20), but the relatively few data on this species foreclose generalizing.

Although continuous reproduction does in fact reduce the seasonal fluctuation in mean length of a population, different growth rates at different times of the year increase this fluctuation once again.

The second sampling year (1974-1975) was warmer than the first one (1973-1974) (Goedmakers, 1980). These different weather conditions in consecutive years may also cause differences in size. Thus both $G$. fossarum and E. berilloni populations attained greater mean cephalic lengths in 1974-1975 than in the previous year (figs. 40 to 43), also at most stations separately (tables IV and V). Of course the percentage of adults in a population may again obscure our data, but can not explain this fact by itself.

Both stations $2 a$ and 7 show less sexual activity in $G$. fossarum (table IV) in the second year, but at the same time a larger mean cephalic length in the total population as well as in ovigerous females only. A similar situation is found at stations 6 and 8 in $E$. berilloni (table V): less and about the same sexual activity, respectively, with the same and a larger mean cephalic length, respectively, of both the total population and the ovigerous females in the second sampling year. We have to conclude 
TABLE V

Summary of data on mean size and sexual activity of $E$. berilloni at the different sampling stations in the Slack over the entire sampling period. This table is partly based on data of Teixeira de Mattos (unpubl.).

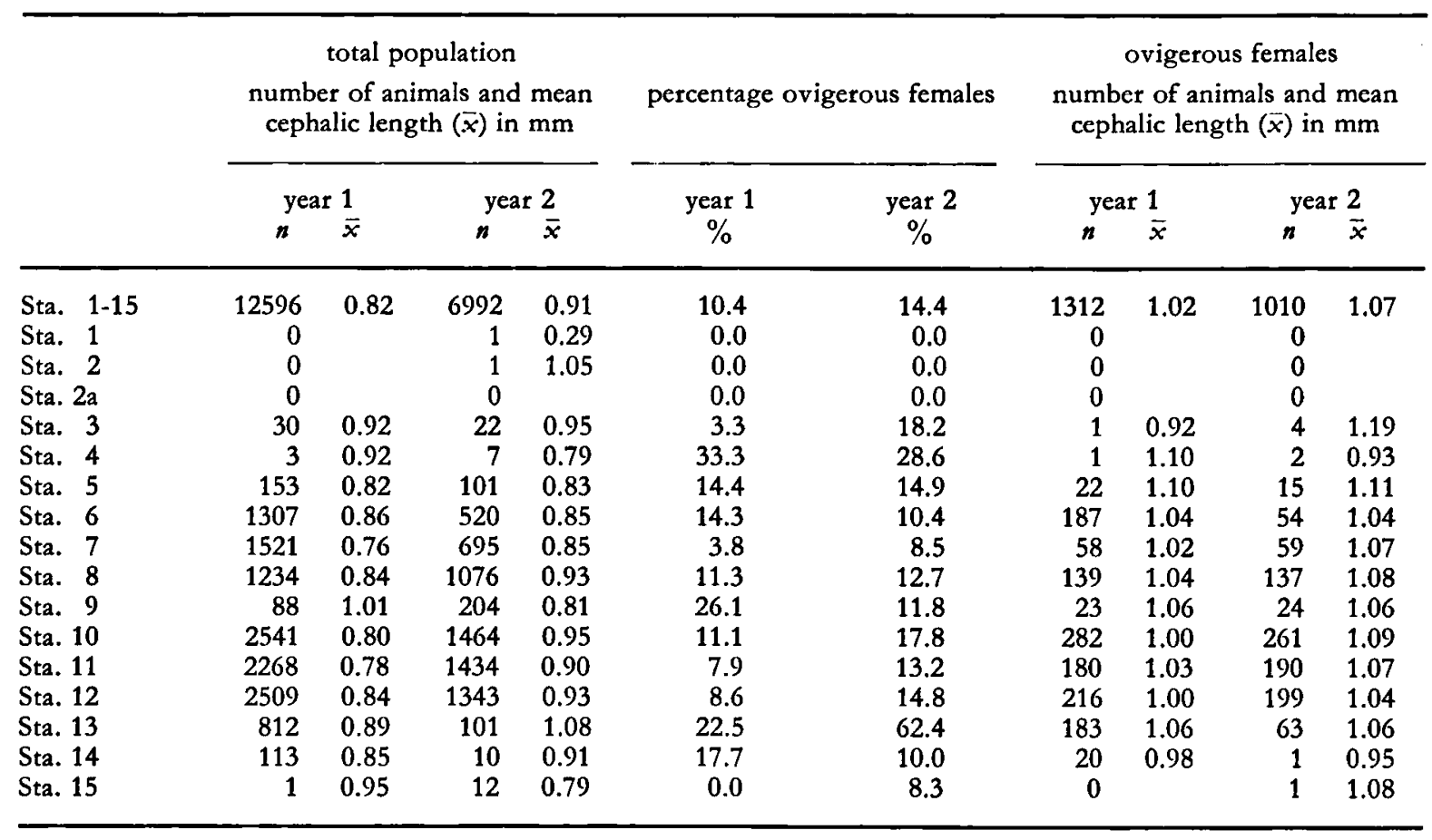

Year 1 summarizes data of the sampling period September 1973 to September 1974; year 2 those of September 1974 to September 1975.

that apart from differences in maturity of a population, differences in growth rates must play a role in size differences between the two years.

Higher water temperatures during the second year have probably caused both a higher sexual activity and a faster growth rate.

\subsubsection{Sexual activity}

The percentage of precopulations is an easily measurable standard for the sexual activity of a population, even in the field. Using this standard has some disadvantages, however. Firstly, it is difficult to identify precopulations of different species in the field. Moreover, during hand-net fishing animals in precopulation come apart as they are handled, especially in periods when the animals are very active. Therefore both the percentage of precopulations (table VI) and the percentage of ovigerous females (figs. 15 and 20 to 36) are used to give an indication of the sexual activity of a population.
The percentage of precopulations (table VI) is not simply correlated with that of ovigerous females. In some cases we find many, in other cases few precopulations compared with the percentage of ovigerous females. Thus, at station 11 each precopulation corresponds to about 2 ovi-

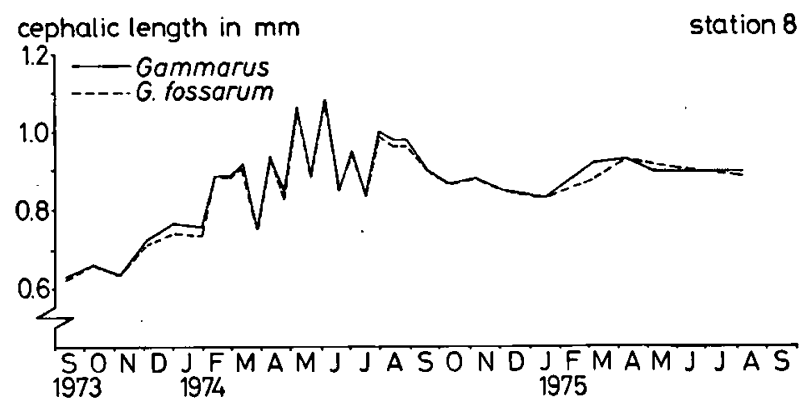

Fig. 37. Seasonal variation in mean size of Gammarus (G. p. pulex $+G$. fossarum), and of $G$. fossarum only, at station 8. Only samples that were fully identified are included in this figure. (Sample size varies between $n=19$ and $n=98$. ) 

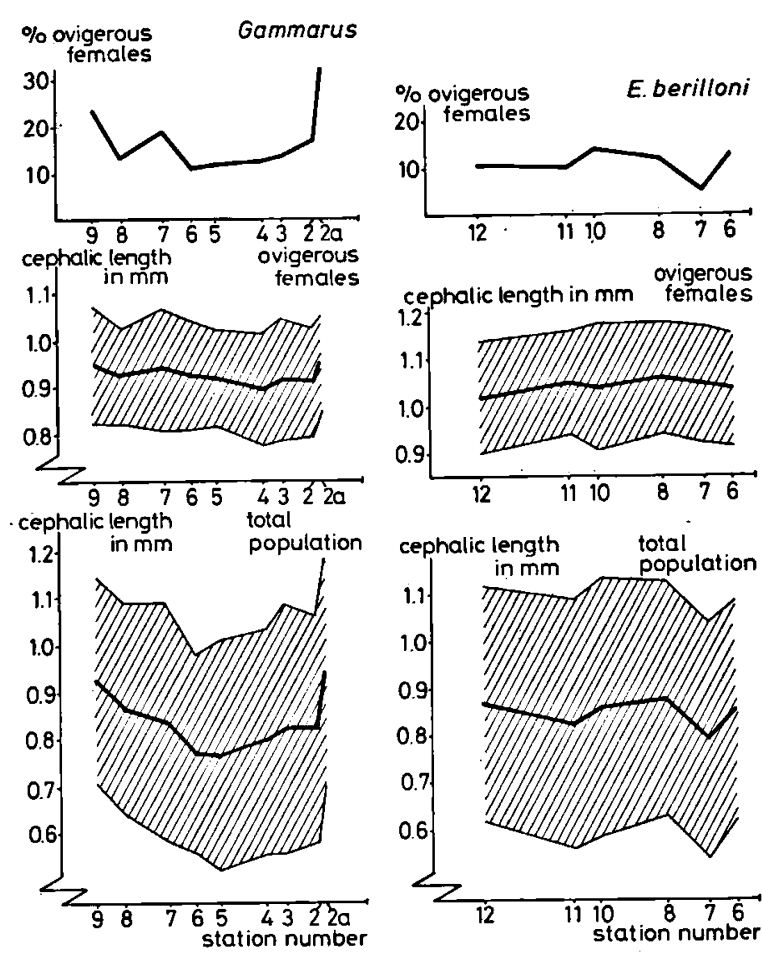

Fig. 38 (left). Microgeographic variation in sexual activity, mean size and its standard deviation of the total Gammarus population ( $n=1520$ to 4412 ) and of ovigerous females only ( $n=200$ to 974 ) over entire sampling period.

Fig. 39 (right). Microgeographic variation in sexual activity, mean size and its standard deviation of the total $E$. berilloni population ( $n=1827$ to 4005$)$ and of ovigerous females only ( $n=117$ to 415$)$ over entire sampling period.

gerous females, while at station $2 a$ each precopulation corresponds to about 6 ovigerous females (mean percentage of precopulations $5.9 \%$ and $5.1 \%$, and mean percentage of ovigerous females $10.1 \%$ and $32 \%$ for station 11 and $2 \mathrm{a}$, respectively.

\subsubsection{Interspecific differences.}

$G$. fossarum shows at some stations a fluctuating (figs. 23 and 27) and at other stations a rather stable (figs. 22 and 24 ) level of sexual activity. $E$. berilloni always has marked peaks in its sexual activity (figs. 31 to 36 ). A similar fluctuating pattern was observed for $G$. $p$. pulex at station 1 (fig. 20).

The mean percentage of ovigerous females over

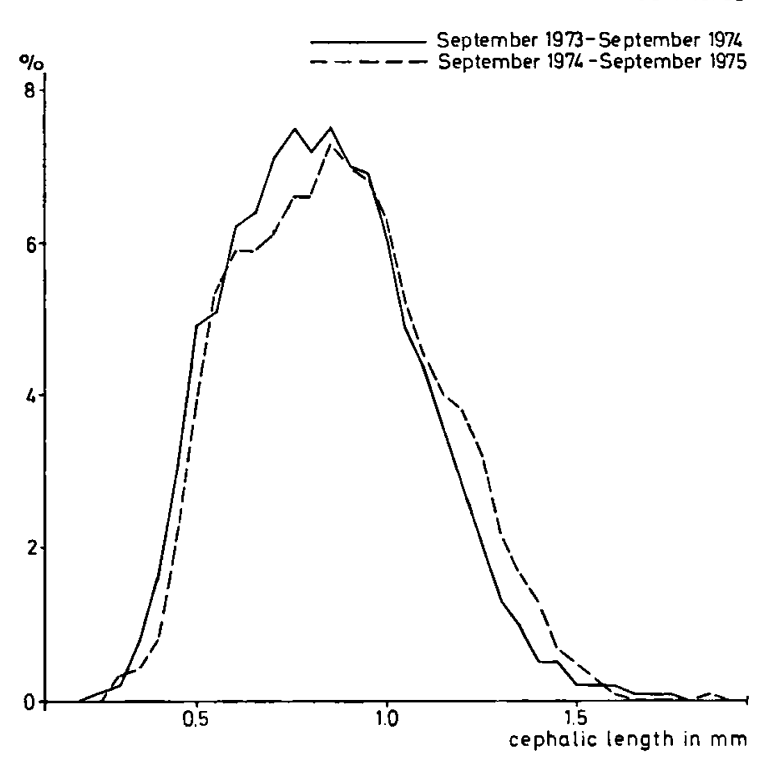

Fig. 40. Between-year difference in size frequencies of Gammarus (all samples involved; $n=20703$ in year 1 and $n=15210$ in year 2 ).

the whole sampling period is larger for Gammarus $(15.6 \%)$ than for E. berilloni (11.9\%). Two alternative explanations are possible. Females of the latter species could be ovigerous during a shorter time or less frequently. The second possibility, that females of $E$. berilloni produce fewer batches of juveniles, has already been concluded in section 5.3 (one or at most two batches during the lifetime of a female $E$. berilloni against two batches for $G$. fossarum in general).

Peaks in sexual activity reach higher levels for E. berilloni (compare figs. 21 to 30 and 31 to 36 ), which is the consequence of the more periodical reproduction of this species.

Since precopulations were not identified, only the data for stations 2 to 5 and stations 10 to 12 should be consulted in table VI to compare the percentage of precopulations of $G$. fossarum and $E$. berilloni, respectively, because these stations are almost exclusively inhabited by either of these two species. In general we found more precopulations for each ovigerous female in $E$. berilloni (an average per station of 0.4-0.6) than in $G$. fossarum (an average per station of $0.2-0.4$ ). Probably $G$. fossarum stays in precopulation during a shorter time than $E$. berilloni. It is also possible that pre- 
TABLE VI

Summary of data on sexual activity at the different sampling stations in the Slack over the entire sampling period. Results for those stations, where the standing crop consists almost entirely of either Gammarus or E. berilloni are printed in bold type. The percentage precopulations of those samples in which precopulae were counted is averaged over the whole sampling period for each station. It is compared with the percentage of all animals captured at a certain station, that was ovigerous.

\begin{tabular}{|c|c|c|c|c|c|c|c|c|}
\hline & \multicolumn{5}{|c|}{ percentage precopulations } & \multicolumn{3}{|c|}{ percentage ovigerous females } \\
\hline & $\begin{array}{c}\text { number of } \\
\text { samples }\end{array}$ & mean & SD & $\min$. & $\max$ & Gammarus & E. berilloni & $\begin{array}{c}\text { total } \\
\text { gammarid } \\
\text { population }\end{array}$ \\
\hline Sta. 1 & 29 & 4.0 & 2.4 & 0.0 & 10.2 & 10.0 & 0.0 & 10.0 \\
\hline Sta. 2 & 30 & 5.3 & 3.1 & 0.0 & 12.4 & 16.6 & 0.0 & 16.6 \\
\hline Sta. $2 a$ & 23 & 5.1 & 9.4 & 0.0 & 20.6 & 32.0 & 0.0 & 32.0 \\
\hline Sta. 3 & 30 & 5.9 & 3.5 & 0.9 & 14.3 & 13.4 & 9.6 & 13.4 \\
\hline Sta. 4 & 30 & 4.7 & 4.0 & 0.0 & 18.1 & 12.3 & 30.0 & 12.3 \\
\hline Sta. 5 & 31 & 3.7 & 2.7 & 0.0 & 9.4 & 11.6 & 14.6 & 11.7 \\
\hline Sta. 6 & 30 & 3.0 & 2.5 & 0.0 & 8.9 & 11.0 & 13.2 & 11.9 \\
\hline Sta. 7 & 32 & 5.0 & 3.4 & 0.0 & 16.2 & 18.9 & 5.3 & 11.8 \\
\hline Sta. 8 & 27 & 4.0 & 3.1 & 0.0 & 13.2 & 13.2 & 11.9 & 12.4 \\
\hline Sta. 9 & 32 & 6.6 & 4.0 & 0.0 & 15.8 & 23.3 & 16.1 & 22.7 \\
\hline Sta. 10 & 29 & 5.3 & 5.4 & 0.0 & 20.0 & 9.6 & 13.6 & 13.3 \\
\hline Sta. 11 & 26 & 5.9 & 4.8 & 0.0 & 19.6 & 16.1 & 10.0 & 10.1 \\
\hline Sta. 12 & 28 & 4.4 & 3.0 & 0.0 & 10.8 & 17.7 & 10.8 & 10.9 \\
\hline
\end{tabular}

copulations of the former species are more easily disturbed.

\subsubsection{Microgeographic variation.}

Of the three species, $G$. fossarum in particular shows great differences in sexual activity between distinct stations (see for instance fig. 44). Percentages of ovigerous females and mean percentages of precopulations of $G$. fossarum vary largely throughout the river Slack (tables IV and VI and fig. 38).

In section 5.3.1.2 the differences between population structures at different stations have already been discussed. We stated that these could not be explained by supposing a preference of certain age groups for certain stations. This means that a low mean percentage of sexual activity at a certain station can not be caused by the presence of many juveniles alone, although the percentage of adult animals in a population certainly affects the level of sexual activity.

Differences in sexual activity between distinct stations have also been found in $E$. berilloni, although less manifest (see for instance fig. 45).

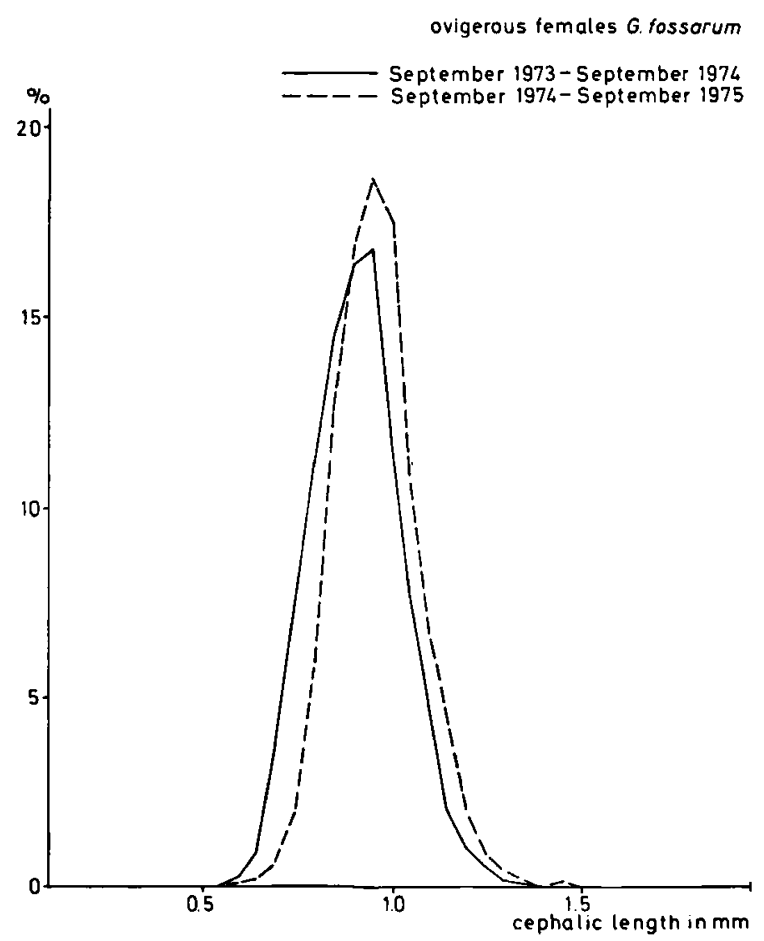

Fig. 41. Between-year difference in size frequencies of ovigerous females of $G$. fossarum (all samples involved; $n=$ 2960 in year 1 and $n=2298$ in year 2 ). 


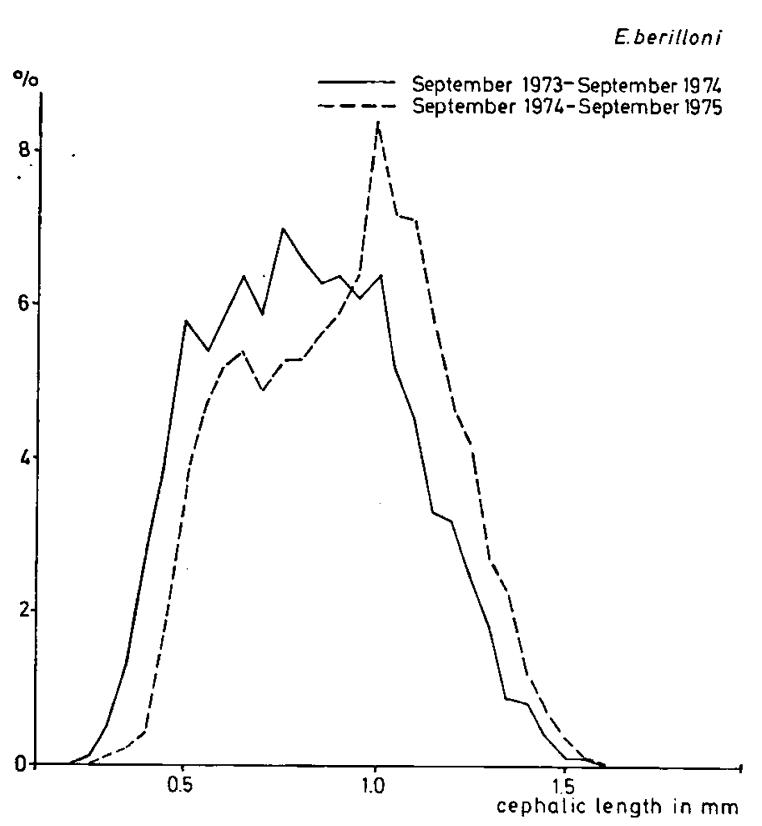

Fig. 42. Between-year difference in size frequencies of $E$. berilloni (all samples involved; $n=12596$ in year 1 and $n=6992$ in year 2).

ovigerous females E. berilloni

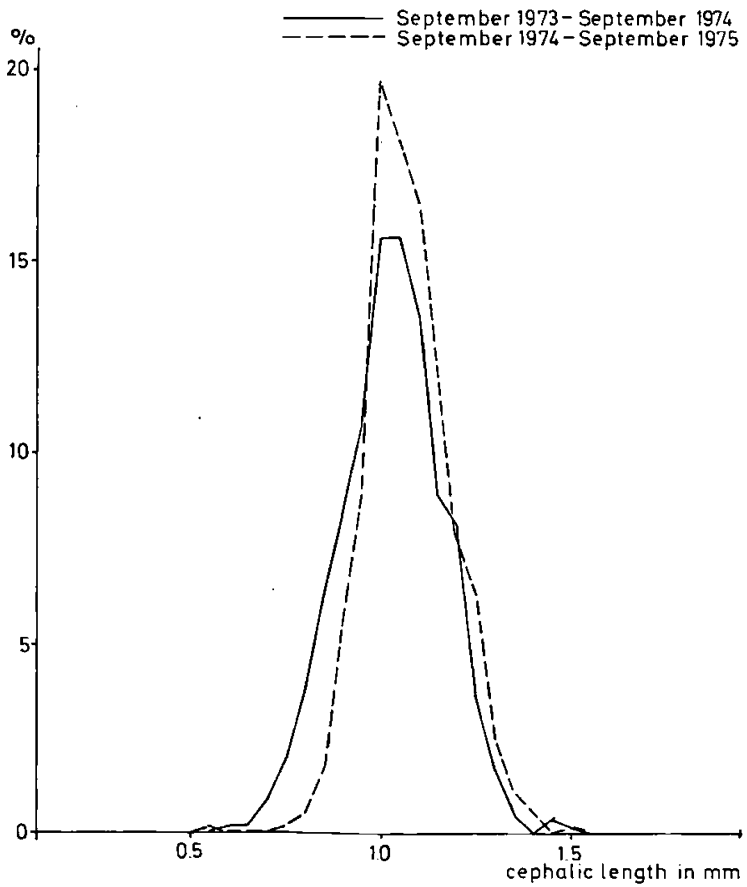

Fig. 43. Between-year difference in size frequencies of ovigerous females of $E$. berilloni (all samples involved; $n=$ 1312 in year 1 and $n=1010$ in year 2 ).
Tables V and VI and fig. 39 show the microgeographic variation in sexual activity of this species.

Fig. 15 provides data on the mean percentage of ovigerous females of only those standing crop samples that were fully identified, and shows a variation in sexual activity for G. p. pulex at different stations. Since the results for $G$. p. pulex are based on a very low percentage of this species found at all stations except for station 1, the microgeographic variation in its sexual activity has to be investigated more thoroughly, before conclusions can be drawn.

\subsubsection{Seasonal and annual variation.}

Apart from a normal, seasonal variation in sexual activity, which was most evident in $E$. berilloni, there is also a marked difference between various years (tables IV and V).

Especially in $E$. berilloni we observed a higher sexual activity (table V: 10.4 and $14.4 \%$ ovigerous females in the first and the second year, respectively) and shorter inactive periods (figs. 31 to 36) during the second sampling year (19741975) than during the first year (1973-1974). Although less pronounced, this phenomenon also occurs in G. fossarum (table IV and figs. 21 to 30).

\subsubsection{Sex ratio}

At a certain stage in their development, juveniles differentiate into males and females. Both mean and maximum size of males are always larger than those of females in the gammarid species studied. Since we found no evidence that males live longer (large males and females disappear from our samples at the same time), the male growth rate must be greater than that of females. This holds true as well for such juveniles as differentiate into males later on, since also the minimum size of recognizable males is larger than that of females. This fact might have affected our data on sex ratios, since smaller animals are more difficult to catch and to identify. Consequently, the numbers of females found may be too low and therefore the sex ratios (males/females) computed might be too high. 
TABLE VII

Summary of data on sex ratio at the different sampling stations in the Slack over the entire sampling period. Only those samples are included that have been fully identified and sexed by the same investigator.

\begin{tabular}{|c|c|c|c|c|c|c|c|c|c|}
\hline & \multicolumn{3}{|c|}{ G.p.pulex } & \multicolumn{3}{|c|}{ G. fossarum } & \multicolumn{3}{|c|}{ E. berilloni } \\
\hline & $\begin{array}{c}\text { number of } \\
\text { samples }\end{array}$ & $\begin{array}{c}\text { mean } \\
\text { sex ratio }\end{array}$ & $\begin{array}{c}\mathrm{SD} \\
\text { sex ratio }\end{array}$ & $\begin{array}{c}\text { number of } \\
\text { samples }\end{array}$ & $\begin{array}{c}\text { mean } \\
\text { sex ratio }\end{array}$ & $\begin{array}{c}\mathrm{SD} \\
\text { sex ratio }\end{array}$ & $\begin{array}{c}\text { number of } \\
\text { samples }\end{array}$ & $\begin{array}{l}\text { mean } \\
\text { sex ratio }\end{array}$ & $\begin{array}{c}\mathrm{SD} \\
\text { sex ratio }\end{array}$ \\
\hline Sta. 1-12* & & & & & 0.96 & 0.15 & & 0.87 & 0.11 \\
\hline Sta. 1 & 10 & 1.39 & 0.41 & 11 & 1.02 & 0.25 & & & \\
\hline Sta. 2 & & & & 6 & 0.91 & 0.25 & & & \\
\hline Sta. $2 a$ & & & & 5 & 0.73 & 0.17 & & & \\
\hline Sta. 3 & & & & 5 & 1.25 & 0.22 & & & \\
\hline Sta. 4 & & & & 5 & 1.01 & 0.14 & & & \\
\hline Sta. 5 & & & & 5 & 1.04 & 0.30 & & & \\
\hline Sta. 6 & & & & 5 & 1.07 & 0.37 & 5 & 0.70 & 0.26 \\
\hline Sta. 7 & & & & 4 & 0.81 & 0.13 & 4 & 0.87 & 0.15 \\
\hline Sta. 8 & & & & 6 & 0.87 & 0.28 & 7 & 1.03 & 0.24 \\
\hline Sta. 9 & & & & 5 & 0.91 & 0.37 & & & \\
\hline Sta. 10 & & & & & & & 5 & 0.79 & 0.20 \\
\hline Sta. 11 & & & & & & & 6 & 0.91 & 0.10 \\
\hline Sta. 12 & & & & & & & 5 & 0.91 & 0.17 \\
\hline
\end{tabular}

* The results of all stations, as represented in this table, are averaged to get an approximation for the mean sex ratio of a certain species in the Slack.

\subsubsection{Interspecific differences.}

In the Slack the mean sex ratio for $E$. berilloni is less than 1. For G. p. pulex this ratio is distinctly higher than 1 . The mean sex ratio of $G$. fossarum is about 1 , but shows clear differences (from 0.73 to 1.25 ) at various stations (table VII). Sex ratio of G. p. pulex is larger than that of G. fossarum even at station 1, where both species coexist (Mann-Whitney test: significance $<1 \%$ ). We found no correlation between mean sex ratio and mean cephalic length of $G$. fossarum or $E$. berilloni populations at different stations.

\subsubsection{Microgeographic variation.}

The sex ratio of $G$. fossarum (table VII and fig. 47) varies along the river (Kruskal-Wallis test: significance $<1 \%$ ). G. fossarum has the highest sex ratio in the centre of its distribution area (station 3 ) and minima at stations 2, 2a and 7 to 9 (minima and maxima differ according to the Mann-Whitney test with a significance of $<1 \%$ ).

A microgeographic variation in sex ratio of $E$. berilloni (table VII and fig. 46) is less clear (Kruskal-Wallis test: not significant). However,

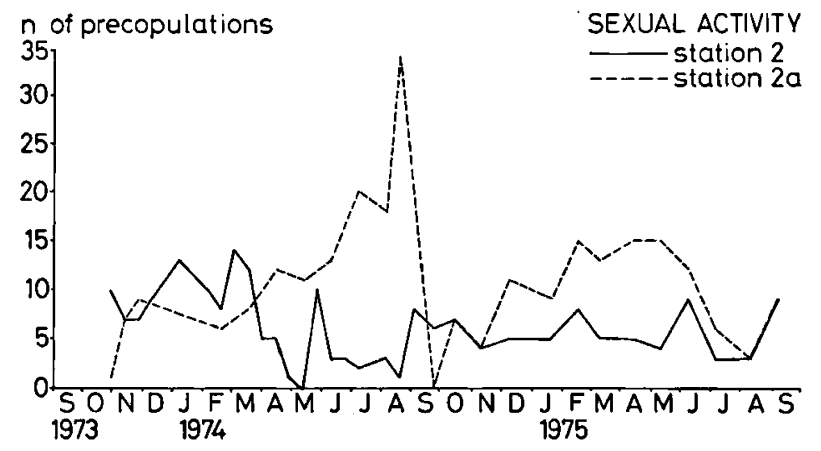

Fig. 44. Seasonal variation in sexual activity (number of precopulations counted in the field) at stations 2 and 2 a. (These stations are mainly inhabited by $G$. fossarum.)

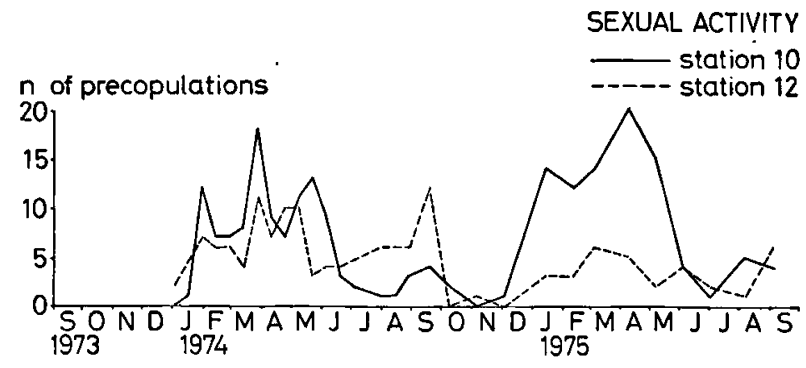

Fig. 45. Seasonal variation in sexual activity (number of precopulations counted in the field) at stations 10 and 12 . (These stations are mainly inhabited by $E$. berilloni.) 

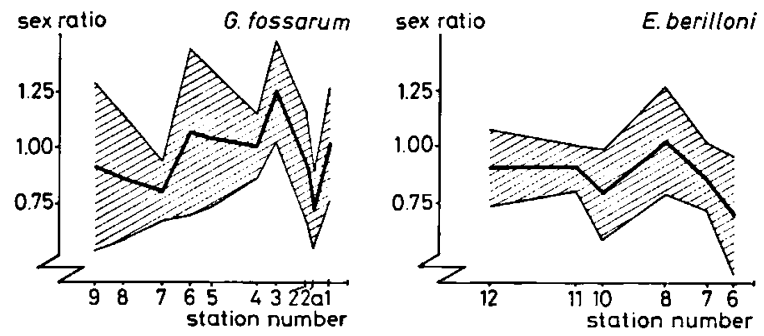

Fig. 46 (left). Microgeographic variation in mean sex ratio and its standard deviation of $G$. fossarum (only those samples that were fully identified and sexed by the same person are included in the figure).

Fig. 47 (right). Microgeographic variation in mean sex ratio and its standard deviation of $E$. berilloni (only those samples that were fully identified and sexed by the same person are included in the figure).

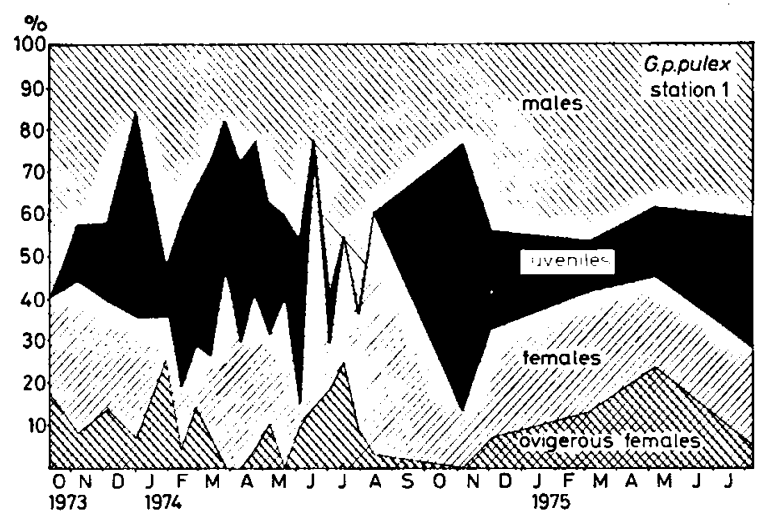

Fig. 48. Seasonal variation in the occurrence of males, (ovigerous) females and juveniles of $G$. fossarum at station 4. (Sample size varies between $n=10$ and $n=96$ ).

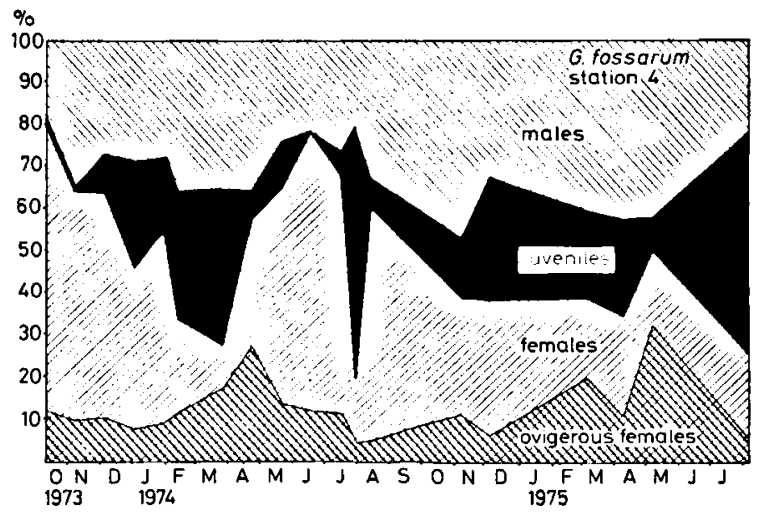

Fig. 49. Seasonal variation in the occurrence of males, (ovigerous) females and juveniles of $G$. fossarum at station è. (Sample size varies between $n=88$ and $n=287$.) the sex ratio of $E$. berilloni at station 8 is larger than that at station 6 (Mann-Whitney test: significance $5 \%$ ). More research is necessary to verify whether the sex ratio of $E$. berilloni varies along the river.

\subsubsection{Seasonal and annual variation.}

A seasonal variation in the percentage of males is evident for both $G$. fossarum and $E$. berilloni (figs. 49 and 50). The fluctuations in the percentages of females of $E$. berilloni and $G$. fossarum and of both sexes of G. p. pulex (fig. 48) are far less regular. The peak in the percentage of female $G$. fossarum (fig. 49) in June 1974 directly after the peak for males, might indicate (1) that male-juveniles obtain sexual characteristics in an earlier stage than female-juveniles, or (2) that environmental conditions are at first favourable for the development of males, and for females only later on. The first hypothesis seems not very probable: the appearance of genital papillae at an earlier stage, together with the larger minimum size of males, would necessitate much faster growth rates for males than for females.

Our results do not allow us to provide final answers on the seasonal variation of sex ratios. Further investigations would be necessary. With the present data in mind, one would expect to find the same pattern of sex ratios for different years, with a marked seasonal fluctuation.

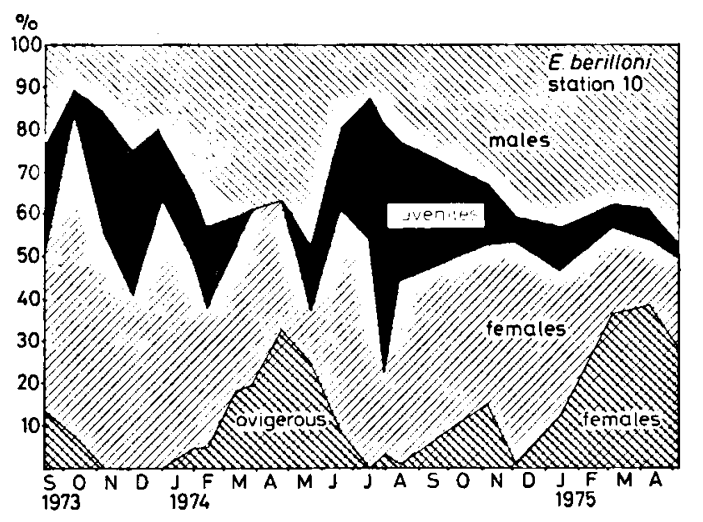

Fig. 50. Seasonal variation of the occurrence of males, (ovigerous) females and juveniles of $E$. berilloni at station 10 . (Sample size varies between $n=88$ and $n=183$ ). 


\subsubsection{Fecundity}

In section 3.4 we stated that fecundity depends on species, age, egg stage and environmental conditions. As females grow older, fecundity increases (fig. 51). The relation between cephalic length and fecundity is linear (exponential regression analyses yielded lower correlation coefficients, see table $\mathrm{X}$ for instance). The large variation in fecundity at each cephalic length (fig. 51) can be partly attributed to the seasonal variation in the correlation between cephalic length and fecundity.

We could find no evidence for a drastic loss or removal of eggs out of the broodpouch during their development (table VIII) in our gammarid species. The number of fully developed juveniles (stage V), however, was always relatively low. This was to be expected since some of the juveniles may already have hatched. Therefore we left out all data concerning ovigerous females carrying brood of stage $\mathrm{V}$ in our following results.

Table VIII shows that $E$. berilloni females steadily lose eggs during the development of their eggs, although this loss is greatest when they reach stage $V$. Fecundity of $G$. fossarum increases after stage I and diminishes again in stage $\mathrm{V}$. This may be caused by the phenomenon that certain females lose all their eggs in an early stage (see also Goedmakers \& Roux, 1975, who found this phenomenon for unfertilized eggs). Results for G. $p$. pulex are based on too few data to permit conclusions. A process as in $G$. fossarum seems likely.

\section{TABLE VIII}

Fecundity for the five egg developmental stages at the mean cephalic length of the ovigerous females of each species. (Fecundity is computed by inserting this mean cephalic length in the formulas that result when cephalic length and number of eggs are linearly correlated for each species separately.)

\section{G.p.pulex G. fossarum E. berilloni}

\begin{tabular}{lrrr} 
Stage I & 13.8 & 11.3 & 31.0 \\
Stage II & ) & 13.6 & 31.7 \\
Stage III & \multirow{2}{*}{19.7} & 11.3 & 27.3 \\
Stage IV & & 11.8 & 25.9 \\
Stage V & 6.6 & 5.5 & 10.7 \\
\hline
\end{tabular}
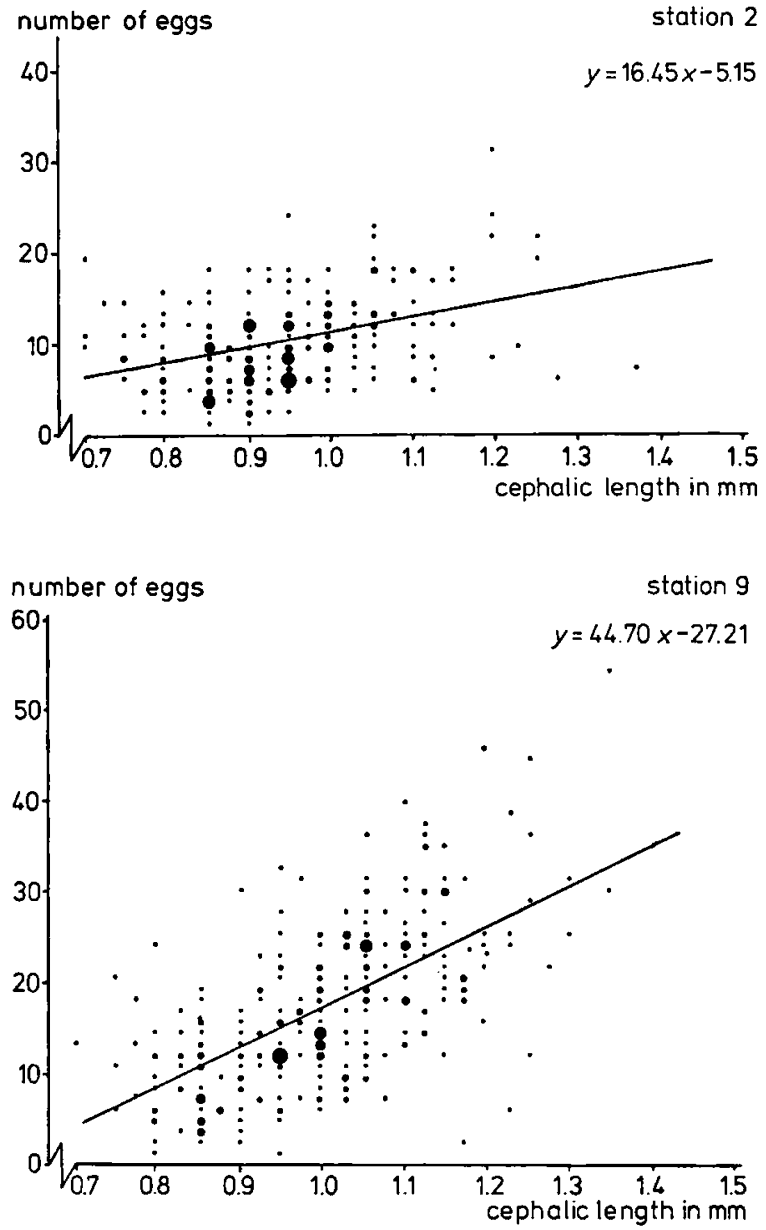

Fig. 51. Relation between fecundity (the number of eggs in stage I to IV an ovigerous female carries in her broodpouch) of $G$. fossarum females and their size over entire sampling period. Upper half of this figure represents data for station $2(r=0.37$, significance $r=0.00001)$, lower half those for station $9(r=0.62$, significance $r=0.00001)$. The surface of the dots is related to the number of times each value occurs.

\subsubsection{Interspecific differences.}

The fecundity/cephalic length ratio is more or less similar for G. p. pulex and G. fossarum (figs. 52 and 53), but female $E$. berilloni of identical lengths carry more eggs (fig. 54). Since in general females of $G$. fossarum are smaller than those of $G$. p. pulex, the latter species as a rule produces more eggs per female than the former. The fecundity at the mean cephalic length of ovigerous females of each species is 14.9 for G. p. pulex, 11.1 for G. fossarum and 28.8 for E. berilloni. 
TABLE IX

Summary of data on fecundity (the number of eggs in stage I to IV an ovigerous female carries in her broodpouch) at the different sampling stations over the entire sampling period. This table is partly based on data of Blom (unpubl.) and Ravenstein (unpubl.).

A. G. p. pulex

\begin{tabular}{|c|c|c|c|c|c|c|c|c|c|}
\hline & \multirow{2}{*}{$\begin{array}{c}n \text { of } \\
\text { ovigerous } \\
\text { females of } \\
\text { which eggs } \\
\text { were counted }\end{array}$} & \multirow{2}{*}{$\begin{array}{c}\text { mean } \\
\text { fecundity } \\
(f)\end{array}$} & \multicolumn{4}{|c|}{$f=b x+a$} & \multirow{2}{*}{$\begin{array}{c}\text { mean cephalic } \\
\text { length }(\bar{x}) \\
\text { ovigerous females } \\
\text { of which eggs } \\
\text { were counted }\end{array}$} & \multirow{2}{*}{$\begin{array}{l}\text { mean cephalic } \\
\text { length all } \\
\text { ovigerous } \\
\text { females }\end{array}$} & \multirow{2}{*}{$\begin{array}{l}\text { mean } \\
\text { ratio } \\
f / x\end{array}$} \\
\hline & & & $a$ & $b$ & $r$ & $\begin{array}{c}\text { significance } \\
r\end{array}$ & & & \\
\hline ta. $1-1$ & 45 & 15.3 & -14.9 & 26.0 & 0.37 & 0.0062 & 1.16 & 1.15 & 12.0 \\
\hline Sta. 1 & 13 & 18.0 & -43.4 & 50.4 & 0.42 & 0.076 & 1.22 & 1.16 & 13.4 \\
\hline Sta. $2-15$ & 32 & 14.2 & -8.5 & 20.0 & 0.35 & 0.026 & 1.14 & 1.13 & 11.4 \\
\hline
\end{tabular}

B. G. fossarum

\begin{tabular}{|c|c|c|c|c|c|c|c|c|c|}
\hline Sta. 1-15 & 1513 & 11.8 & -13.6 & 26.6 & 0.49 & 0.00001 & 0.96 & 0.93 & 11.0 \\
\hline Sta. 1 & 23 & 12.5 & & & & & 1.07 & 0.95 & 10.6 \\
\hline Sta. 2 & 259 & 10.3 & -4.5 & 16.8 & 0.39 & 0.00001 & 0.93 & 0.91 & 9.8 \\
\hline Sta. 2a & 224 & 10.1 & -7.3 & 18.1 & 0.38 & 0.00001 & 0.96 & 0.95 & 9.3 \\
\hline Sta. 3 & 231 & 11.4 & -8.6 & 21.0 & 0.48 & 0.00001 & 0.95 & 0.91 & 10.7 \\
\hline Sta. 4 & 94 & 9.8 & -4.2 & 15.7 & 0.38 & 0.00009 & 0.89 & 0.89 & 9.7 \\
\hline Sta. 5 & 113 & 9.5 & -7.2 & 17.7 & 0.39 & 0.00001 & 0.94 & 0.92 & 8.9 \\
\hline Sta. 6 & 47 & 9.3 & -15.0 & 25.6 & 0.55 & 0.00003 & 0.95 & 0.93 & 8.4 \\
\hline Sta. 7 & 98 & 11.9 & -10.6 & 23.9 & 0.50 & 0.00001 & 0.94 & 0.94 & 11.3 \\
\hline Sta. 8 & 45 & 12.0 & -22.5 & 36.6 & 0.63 & 0.00001 & 0.94 & 0.93 & 11.4 \\
\hline Sta. 9 & 289 & 17.5 & -27.2 & 44.7 & 0.62 & 0.00001 & 1.00 & 0.95 & 16.0 \\
\hline Sta. 10 & 9 & 11.1 & & & & & 0.98 & 0.93 & 9.8 \\
\hline Sta. 11 & 6 & 10.8 & & & & & 0.82 & 0.92 & 12.7 \\
\hline Sta. 12 & 2 & 12.0 & & & & & 0.94 & 0.99 & 12.0 \\
\hline \multicolumn{10}{|c|}{ C. E. berilloni } \\
\hline Sta. $1-15$ & 703 & 30.3 & -42.0 & 67.9 & 0.51 & 0.00001 & 1.07 & 1.04 & 27.0 \\
\hline Sta. 3 & 2 & 26.0 & & & & & 0.99 & 1.14 & 25.0 \\
\hline Sta. 4 & - & - & & & & & - & 0.99 & - \\
\hline Sta. 5 & 3 & 27.3 & & & & & 1.03 & 1.10 & 25.3 \\
\hline Sta. 6 & 23 & 25.0 & -45.0 & 64.3 & 0.76 & 0.00002 & 1.09 & 1.04 & 21.7 \\
\hline Sta. 7 & 23 & 24.6 & -37.1 & 59.2 & 0.52 & 0.0053 & 1.04 & 1.05 & 22.0 \\
\hline Sta. 8 & 77 & 29.0 & -70.5 & 91.9 & 0.63 & 0.00001 & 1.08 & 1.06 & 25.1 \\
\hline Sta. 9 & 16 & 39.0 & -105.2 & 132.2 & 0.94 & 0.00001 & 1.09 & 1.06 & 33.1 \\
\hline Sta. 10 & 208 & 30.2 & -34.6 & 60.8 & 0.51 & 0.00001 & 1.07 & 1.04 & 26.9 \\
\hline Sta. 11 & 138 & 27.1 & -29.4 & 53.3 & 0.37 & 0.00001 & 1.06 & 1.05 & 24.2 \\
\hline Sta. 12 & 158 & 31.6 & -36.0 & 64.9 & 0.50 & 0.00001 & 1.04 & 1.02 & 29.0 \\
\hline Sta. 13 & 55 & 39.1 & -44.2 & 74.3 & 0.49 & 0.00007 & 1.12 & 1.06 & 33.3 \\
\hline
\end{tabular}

5.3.4.2. Microgeographic variation.

Females of G. p. pulex living at station 1 show a distinctly faster increase of fecundity when they grow larger (fig. 52) than females living at other stations, where this species occurs only sporadically. Differences in mean fecundity, however, are less marked (table IX A), since the mean cephalic length of ovigerous females lies near the point where the two lines of fig. 52 cross.

In $G$. fossarum fecundity gradually increases downstream (table IX B and fig. 55). Therefore the difference in fecundity between G. p. pulex and $G$. fossarum found in section 5.3.4.1 is even more explicit at station 1 , since in this part of their distribution area fecundity of $G$. p. pulex reaches a comparatively high value and that of 
G. fossarum a rather low one. This downstream increasing fecundity might be caused by the increase of fecundity with animal size, which shows a steeper slope going downstream (fig. 53).

$E$. berilloni equally shows a downstream increasing fecundity (table IX C and fig. 56). The variation in fecundity, however, is very large. A special sampling program, which excludes for instance seasonal variation, would be needed to test the hypothesis of a downstream increasing fecundity.

E. berilloni was only sporadically found at station 9, therefore the peak in fecundity (table IX C) at this station reflects data of few animals only. For this reason we did not include the results for station 9 in fig. 56. The peak in fecundity at station 9 could not be attributed to aberrant cephalic lengths (see data on number of eggs/ cephalic length in table IX $C$ ), but was caused by the striking, highly significant relation between cephalic length and fecundity, which showed a very steep slope (fig. 54).

\subsubsection{Seasonal and annual variation.}

Not that many data on seasonal variation are available as to allow definite conclusions. In $E$. berilloni (fig. 58) there is nevertheless a vague indication of a seasonal fluctuation in the mean fecundity/cephalic length ratio. Females of the same length slowly acquire more eggs as the spring

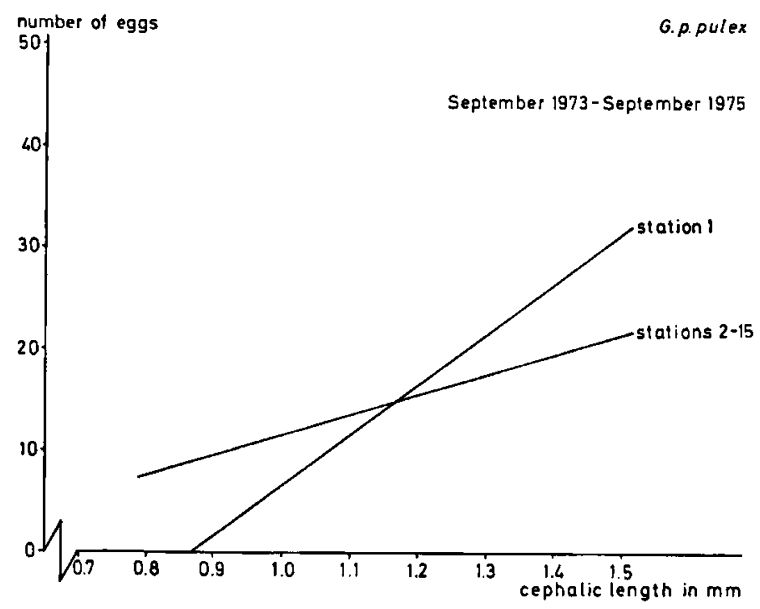

Fig. 52. Relation between fecundity (the number of eggs in stage I to IV an ovigerous female carries in her broodpouch) of G. p. pulex females and their size (see also table IX A).

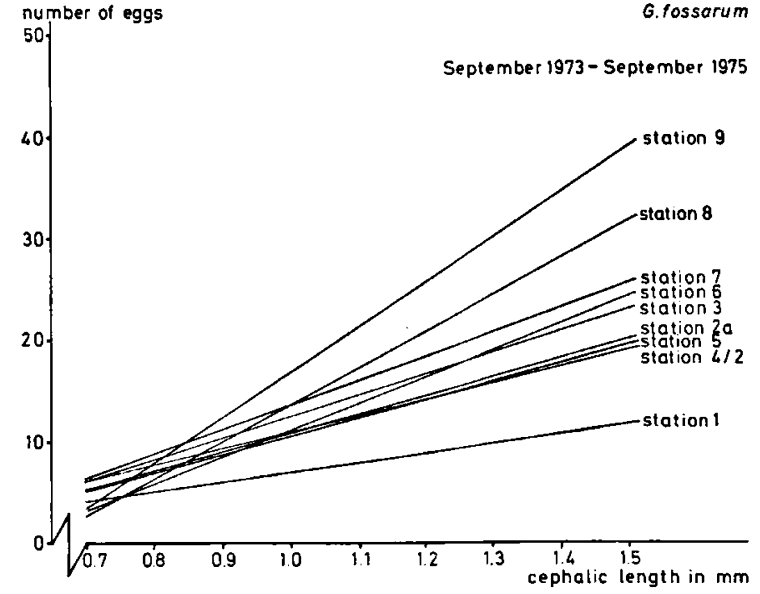

Fig. 53. Relation between fecundity (the number of eggs in stage I to IV an ovigerous female carries in her broodpouch) of $G$. fossarum females and their size (see also table IX B).

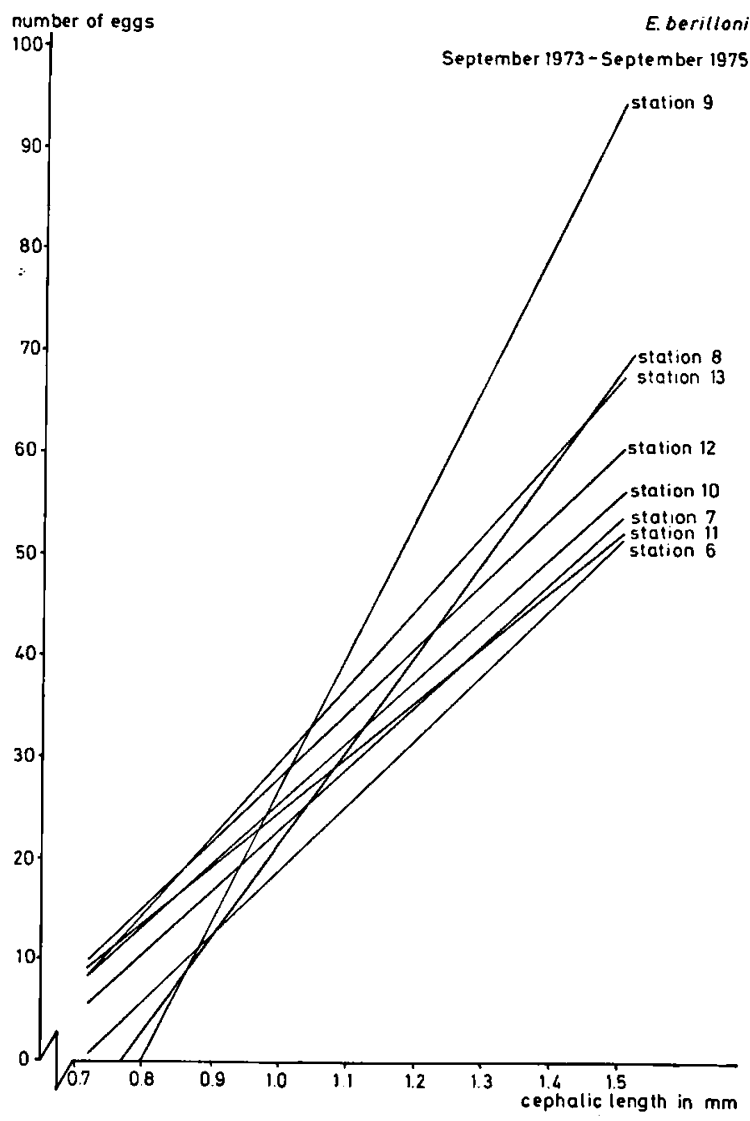

Fig. 54. Relation between fecundity (the number of eggs in stage I to IV an ovigerous female carries in her broodpouch) of $E$. berilloni females and their size (see also table IX C). 

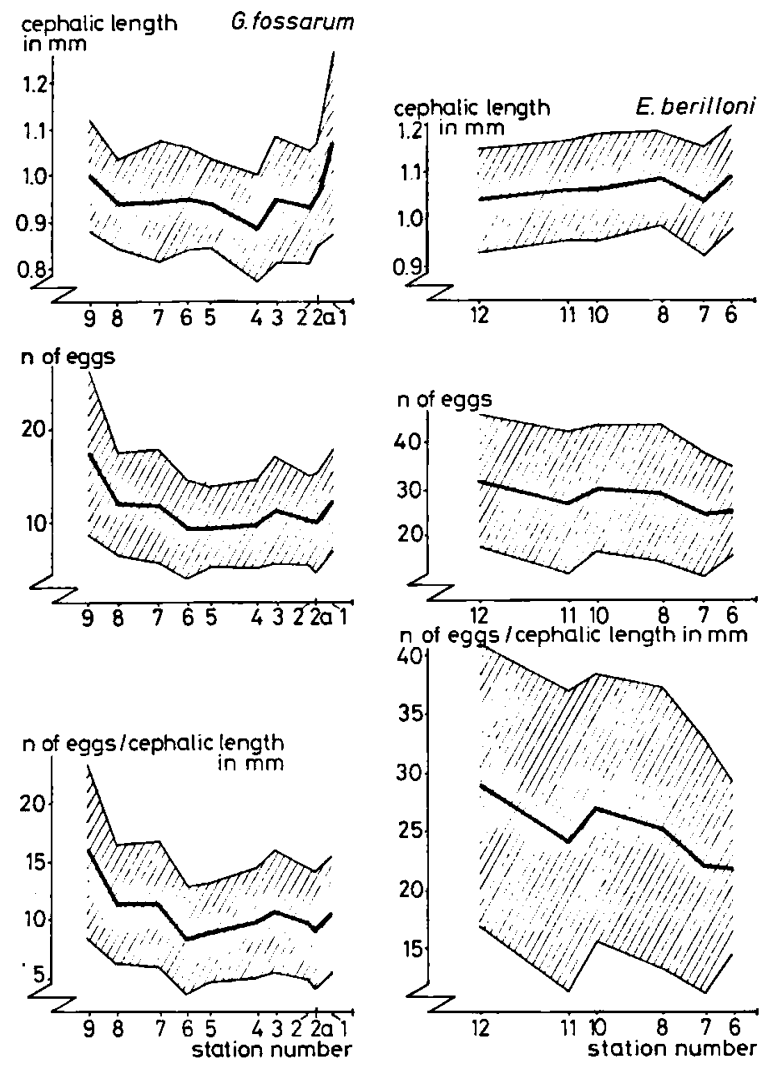

Fig. 55 (left). Microgeographic variation in mean size, mean fecundity, mean ratio fecundity/size and their standard deviation of $G$. fossarum females of which the fecundity was measured. (Sample size varies between $n=23$ and $n=298$.)

Fig. 56 (right). Microgeographic variation in mean size, mean fecundity, mean ratio fecundity/size and their standard deviation of $E$. berilloni females of which the fecundity was measured. (Sample size varies between $n=23$ and $n=208$.)

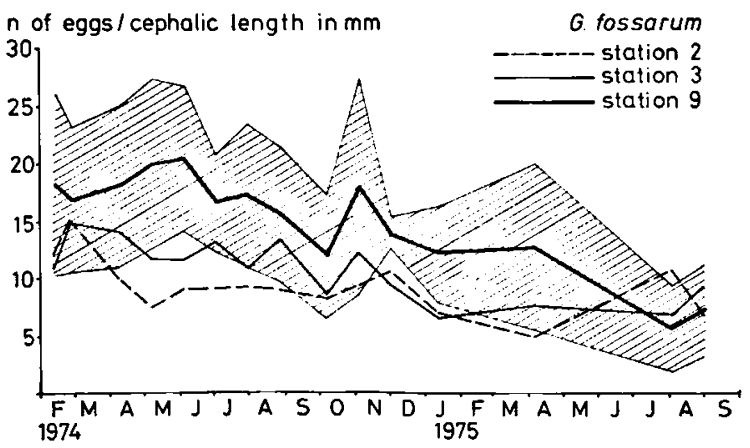

Fig. 57. Seasonal variation in mean ratio fecundity/size of $G$. fossarum females of which the fecundity was measured at stations 2, 3 and 9, with its standard deviation at station 9 (sample size varies between $n=2$ and $n=36$ ). season advances (February to May). This is probably caused by rising water temperatures in this period. A peak in this ratio is reached before the peak in length (compare figs. 34 and 58), which means that maximum numbers of eggs are carried by females of just under maximum length.

The constancy in sexual activity of $G$. fossarum makes our results on fecundity difficult to interpret. At the same time females of different age groups are ovigerous. Animals of a certain length tend to have less eggs in the second half of our sampling period (fig. 57). This might be caused by a correlation between sexual activity and fecundity. More research on fecundity has to be done to provide ultimate answers.

The differences between the two years of investigation seem to be clear (figs. 57,58 and table $\mathrm{X}$ ), but may have been affected by the selection of samples of which fecundity was measured (compare data on mean cephalic length of ovigerous females both in tables IX and X). Both fecundity and the ratio fecundity/cephalic length differ per year. Fecundity was smaller in the second year, because female $G$. fossarum and $E$. berilloni carried less eggs at a certain length (table $\mathrm{X}$ ), and not because of a higher amount of small females in the second year.

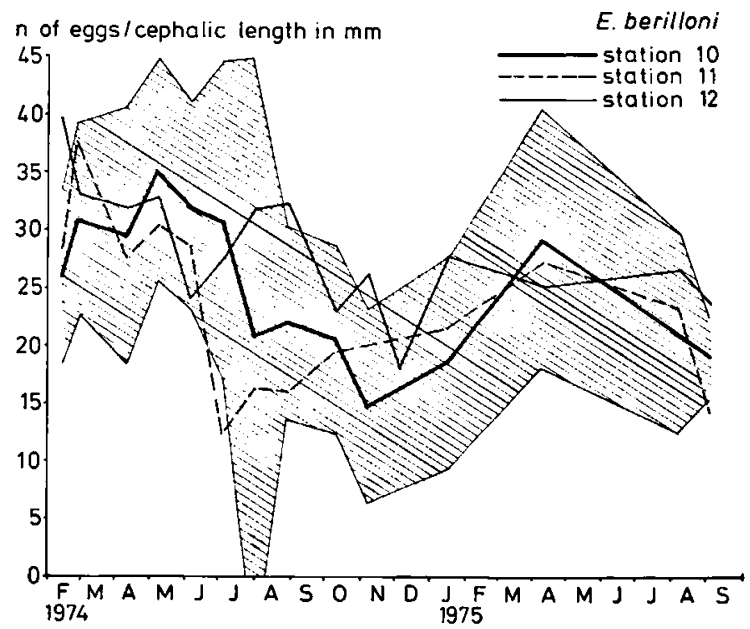

Fig. 58. Seasonal variation in mean ratio fecundity/size of $E$. berilloni females of which the fecundity was measured at stations 10,11 and 12 , with its standard deviation at station 10 (sample size varies between $n=2$ and $n=40$ ). 
TABLE X

Summary of data on fecundity at all sampling stations over sampling year 1 (September 1973-September 1974) and sampling year 2 (September 1974-September 1975) separately.

\begin{tabular}{|c|c|c|c|c|c|c|}
\hline & \multicolumn{2}{|c|}{ G.p.pulex } & \multicolumn{2}{|c|}{ G. fossarum } & \multicolumn{2}{|c|}{ E. berilloni } \\
\hline & year 1 & year 2 & year 1 & year 2 & year 1 & year 2 \\
\hline $\bar{x}$ ovig. ㅇ & 1.09 & 1.20 & 0.91 & 0.96 & 1.02 & 1.07 \\
\hline $\begin{array}{l}\bar{x} \text { ovig. }+{ }^{\prime} \\
\text { (only those of } \\
\text { which fecundity } \\
\text { was measured) }\end{array}$ & 1.14 & 1.18 & 0.97 & 0.94 & 1.07 & 1.06 \\
\hline mean $f$ & 16.7 & 14.4 & 14.6 & 9.5 & 33.5 & 26.4 \\
\hline mean $f / x$ & 13.7 & 10.8 & 13.7 & 8.9 & 28.8 & 22.5 \\
\hline$a$ & & & -16.2 & -7.7 & -40.0 & -39.0 \\
\hline$b$ & & & 31.6 & 18.3 & 68.5 & 61.9 \\
\hline$r$ & & & $0.54 *$ & 0.43 & $0.52 * *$ & 0.49 \\
\hline significance $r$ & & & 0.00001 & 0.00001 & 0.00001 & 0.00001 \\
\hline $\begin{array}{l}f \text { calculated } \\
\quad \text { (from regression } \\
\text { analysis of } f \text { with } x \text {, } \\
\text { and } \bar{x} \text { ovig. } \$ \text { 우) }\end{array}$ & & & 12.6 & 9.9 & 29.8 & 27.3 \\
\hline
\end{tabular}

* After a correlation of $V f$ with $x, \log f$ with $x$, and $\log f$ with $\log x$, the value for $r$ is $0.53,0.48$, and 0.47 , respectively (in all cases the significance is 0.00001 ).

** After a correlation of $V f$ with $x, \log f$ with $x$, and $\log f$ with $\log x$, the value for $r$ is $0.48,0.38$, and 0.37 , respectively (in all cases the significance is 0.00001 ).

\subsubsection{Condition}

Apart from the influences of environmental conditions on the thriving of gammarids, their condition may differ widely. Parasites may seriously affect the condition of an animal, predators may damage them and algae sticking to their legs or silt between their setae may hamper their movements.

\subsubsection{Parasitation.}

G. fossarum and E. berilloni are parasitized by different Acanthocephala species: Polymorphus minutus (Goeze, 1782) and Metechinorbynchus truttae (Schrank, 1788), respectively (Van Maren, 1977). Since we do not know how these parasites affect their life cycles in nature we can not explain differences in life histories by differences in parasitation. There is, however, a marked distinction between various stations (table $\mathrm{XI}$ ), with high rates of parasitation at stations influenced by bioindustries.

\section{TABLE XI}

Summary of data on parasitation. ( $0=$ no animals parasitized; $1=1-3 \%$ of the animals parasitized; $2=4-10 \%$; $3=11-20 \% ; 4=21-40 \% ; 5=41-60 \% ; 6=61-100 \%$ ). No distinction as to species is made.

\begin{tabular}{|c|c|c|c|c|c|}
\hline & $\begin{array}{c}\text { number of } \\
\text { samples in } \\
\text { which } \\
\text { parasitation } \\
\text { was measured }\end{array}$ & mean & $\mathrm{SD}$ & $\min$. & $\max$. \\
\hline Sta. 1 & 16 & 2.7 & 1.5 & 1 & 6 \\
\hline Sta. 2 & 14 & 0.9 & 0.5 & 0 & 2 \\
\hline Sta. $2 a$ & 14 & 1.1 & 0.6 & 0 & 2 \\
\hline Sta. 3 & 19 & 2.3 & 1.1 & 1 & 4 \\
\hline Sta. 4 & 24 & 2.5 & 1.5 & 1 & 5 \\
\hline Sta. 5 & 22 & 2.3 & 1.4 & 0 & 5 \\
\hline Sta. 6 & 20 & 2.2 & 1.5 & 1 & 5 \\
\hline Sta. 7 & 17 & 1.6 & 0.9 & 0 & 3 \\
\hline Sta. 8 & 20 & 1.4 & 1.3 & 0 & 5 \\
\hline Sta. 9 & 25 & 1.3 & 0.6 & 0 & 3 \\
\hline Sta. 10 & 17 & 1.3 & 0.7 & 0 & 2 \\
\hline Sta. 11 & 16 & 0.8 & 0.6 & 0 & 2 \\
\hline Sta. 12 & 16 & 1.4 & 1.0 & 0 & 4 \\
\hline
\end{tabular}




\subsubsection{Damaged animals.}

Especially at station 6 we often found damaged animals. Since we did not develop a criterion for the condition of an animal, we can not compare various stations in an exact way.

We do not know enough on parasitation or other facts that influence the condition of gammarids in the Slack to comment authoritatively on seasonal fluctuations or between-year differences. As was stated in part I of this study (Goedmakers, 1980), food was always amply available.

\subsubsection{Death}

Gammarids die from diseases, predators or of old age like most animals. We do not know anything about diseases, and we expect a different influence of various predators on each life stage. As to age, we estimated the maximal lifetime for all three species (section 5.3) to be one year at most.

We do know, however, the effects of all death causes together on the structure of a population (figs. 40 and 42 ): only a very small percentage of the animals reaches old age.

\subsection{Laboratory experiments}

Since it is very difficult to obtain data on life cycles of individual gammarids in the field, we conducted some laboratory experiments in order to check our conclusions and to complete the data from our field observations. Thus, we followed a great number of females of all three species during the development of their eggs. For this purpose we collected 800 specimens of $G$. fossarum at station 5, 1600 specimens of $G$. p. pulex at station 1 and 1600 specimens of E. berilloni at station 10, all on October 14th, 1975.

The animals were kept in plastic trays of $25 \times$ $25 \mathrm{~cm}$ under $10 \mathrm{~cm}$ water at temperatures of 5,10 or $15^{\circ} \mathrm{C}$. Generally we put 100 animals in one tray, but we had some basins with 200 animals of $G$. p. pulex or E. berilloni. This did not influence our results.

As soon as ovigerous females occurred in the trays, they were isolated, each in its own small basin $(7 \times 7 \mathrm{~cm}$ with $10 \mathrm{~cm}$ deep water) at the the temperature they were kept at originally, until juveniles hatched (for details see Goedmakers, 1972). For every female the number of days between oviposition and hatching of the juveniles was registered. After the hatching of the juveniles the female was removed and juveniles raised until maturity at $15^{\circ} \mathrm{C}$.

\subsubsection{Egg incubation time}

Temperature clearly affects the number of days from oviposition until the hatching of juveniles (table XII). At low temperatures this period is rather long, or no hatching occurs at all.

The slight interspecific differences in egg incubation time at a certain temperature, as observed in our experiments, have to be investigated more thoroughly in order to draw definite conclusions. Some data from literature do not corroborate the presence of differences that are indicated by our experiments (Roux, 1975; Koch-Kallnbach \& Meijering, 1977; Meijering \& Teichmann, 1978). According to these authors instars or periods between two moults of reproducing animals are the same for G. fossarum, G. p. pulex and G. roeseli

TABLE XII

Egg incubation time $(t)$ under laboratory conditions in days, number of animals of which the juveniles hatched and the standard deviation of $t$.

\begin{tabular}{|c|c|c|c|c|c|c|c|c|c|}
\hline & \multicolumn{3}{|c|}{ G.p.pulex } & \multicolumn{3}{|c|}{ G. fossarum } & \multicolumn{3}{|c|}{ E. berilloni } \\
\hline & $n$ & $t$ & SD & $n$ & $t$ & SD & $n$ & $t$ & SD \\
\hline $15^{\circ} \mathrm{C}$ & 28 & 22 & 2.0 & 12 & 24 & 4.2 & 5 & 21 & 3.9 \\
\hline $10^{\circ} \mathrm{C}$ & 3 & 28 & 2.6 & 9 & 31 & 6.8 & 1 & 36 & - \\
\hline $5^{\circ} \mathrm{C}$ & 0 & - & - & 1 & 52 & - & 0 & - & - \\
\hline
\end{tabular}


Gervais, 1835. (Their period between two moults starts with oviposition and ends with the following moult; it is about the same period that we call egg incubation time: the period between oviposition and the hatching of juveniles.)

The season in which sexual maturity is reached might affect the time it takes for eggs to develop. Since we have only data on animals collected in October, we can not elucidate seasonal influence on egg incubation time any further.

As expected, we found no correlation between age, in this case the cephalic length of female G. p. pulex at the time their juveniles hatched, and the incubation time of their eggs $(r=-0.087)$.

\subsubsection{Reproductive success}

Janssen et al. (1979) define reproductive success as the percentage of the average number of eggs in the marsupium that survive as juveniles after the first moult. It is not clear whether a first moult of the juveniles or the first moult after oviposition of their mother is meant. This reproductive success is very difficult to measure. In our experiments the numbers of juveniles produced were too variable to be used in a computation of reproductive success.

However, we can tell something about the reproductive success when defined as the percentage of animals that produces live offspring and the percentage of batches offspring of which at least one of the juveniles reaches maturity. The percentage of animals that produces live offspring is clearly temperature dependent, and different for our three species (table XIII). Our experiments show clearly that $G$. fossarum thrives best at $10^{\circ} \mathrm{C}$, $G$. p. pulex at $15^{\circ} \mathrm{C}$, while E. berilloni produces hardly any of fspring at any of these three temperatures. This indicates a problem in our experimental situation: not all three species react in the same way to the conditions of fered.

The reproductive success of the three species, defined as the percentage of animals that produces live offspring, and the percentage of batches offspring that reaches maturity, show the relative suitability of our experimental conditions for G. p. pulex. These results support our field data on $G$. p. pulex living in an instable environment, and $G$. fossarum living in the stable upper region of the Slack, with low water temperatures.
TABLE XIII

Reproductive success under laboratory conditions in the three gammarid species studied.

\begin{tabular}{|c|c|c|c|c|}
\hline & & $\begin{array}{l}\dot{x} \\
\stackrel{\Xi}{\Xi} \\
\dot{0} \\
\dot{0}\end{array}$ & $\underbrace{5}_{0}$ & 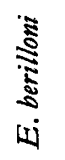 \\
\hline \multirow{3}{*}{$\begin{array}{l}\% \text { of animals that produces } \\
\text { live offspring }\end{array}$} & $5^{\circ} \mathrm{C}$ & - & 1 & - \\
\hline & $10^{\circ} \mathrm{C}$ & 3 & 9 & 1 \\
\hline & $15^{\circ} \mathrm{C}$ & 7 & 6 & 1 \\
\hline $\begin{array}{l}\% \text { of batches offspring of } \\
\text { which at least one of the } \\
\text { juveniles reaches maturity }\end{array}$ & $15^{\circ} \mathrm{C}$ & 42 & 8 & 16 \\
\hline $\begin{array}{l}\% \text { of animals that produces } \\
\text { live offspring of which at } \\
\text { least one juvenile reaches } \\
\text { maturity }\end{array}$ & $15^{\circ} \mathrm{C}$ & 3 & 0.5 & 0.2 \\
\hline
\end{tabular}

It takes about three and a half months at $15^{\circ} \mathrm{C}$ to develop from hatched juvenile until sexually mature adult. Our results do not enable us to decide whether maturation time is different for our three species at the same temperature.

\subsection{Calculated life histories}

Janssen et al. (1979) calculated the length of some life processes of two brackish-water species $G$. aequicauda (Martynov, 1931) and $G$. insensibilis Stock, 1966. Obviously, life processes of our three freshwater species take a much longer time. I do not feel able to give a calculation like Janssen et al. (1979) did, since a lot of necessary data are not yet available.

We have no data about the average life span of our species in the laboratory. At $15^{\circ} \mathrm{C}$ they are sexually mature after 105-120 days, which is about twice as long as Janssen et al. (1969) calculated for the two brackish-water species. The egg incubation time varies for our three species between 21 and 24 days at $15^{\circ} \mathrm{C}$, which is $3-6$ days longer than for the brackish-water species. No exact data on the duration of precopulations are known, but from our laboratory observations we estimate it to be about 6 days at $15^{\circ} \mathrm{C}$, which is three days longer than in the brackish-water species. 
The temperature coefficient, $Q_{10}$, of various life processes seems to be different at any rate for Gammarus and Echinogammarus (cf. table XII). A comparison of water temperatures of the river Slack (Goedmakers, 1980: figs. 13-14) with data in tables XII and XIII illustrates the differences in life cycles (section 5.3) of the three species clearly.

G. fossarum is able to reproduce throughout the year, since water temperatures vary roughly between 5 and $15^{\circ} \mathrm{C}$. The high sexual activity in spring is evidently caused by water temperature values. The higher water temperatures in the second sampling year can account for the higher sexual activity in that period.

Sexual activity of $G$. p. pulex in the laboratory varies largely with temperature. Especially at station 1 , temperatures vary largely during the season, and so does sexual activity of this species.

E. berilloni stops breeding activity after a sharp decline in water temperature. Sexual activity probable increases with increasing daylength, because in the second sampling year sexual activity even rose when mean temperatures were still slowly falling. The relatively short period of inactivity during the second sampling year must probably be attributed to a smaller drop in water tempera-

\section{station 8}
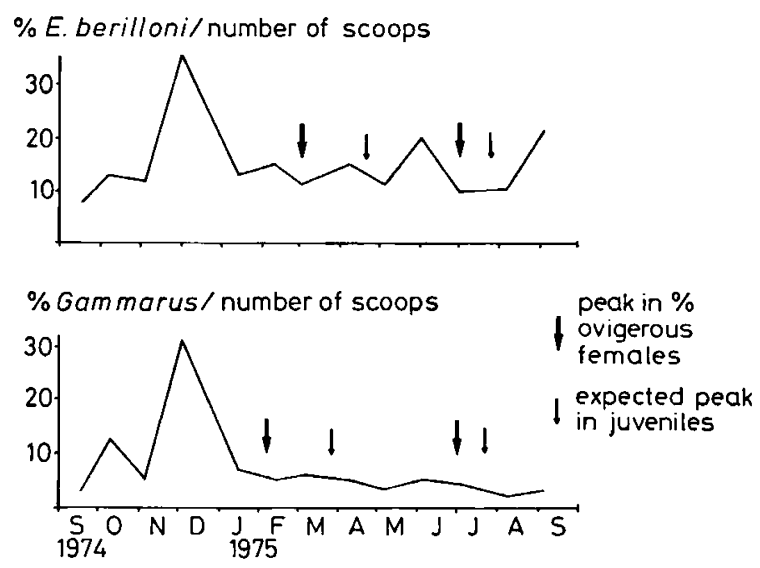

Fig. 59. Seasonal variation in population density of Gam. marus and E. berilloni at station 8. (Population density is computed by dividing the percentage of both Gammarus and $E$. berilloni in a sample by the number of ten second scoops needed to catch one hundred animals.)

Peaks in sexual activity (see also figs. 29 and 33 ) and peaks in the hatching of juveniles (as expected from experiments; see table XII) are indicated by arrows. tures that year, compared with the first sampling year. The actual value of water temperature seems to be important for a process to occur (or not), but changes in water temperature start or stop a process.

A comparison of egg incubation time, peaks in sexual activity and variations in abundance (fig. 59) clearly shows that a sudden rise in population density is not correlated with hatching. In fig. 59 this is illustrated for station 8 , but the same holds true for all other stations (compare data on population density in table II, sexual activity in figs. 20 to 36 and egg incubation time in table XII). This means that rises in population density can not be explained by population explosions. In my opinion crowding or dispersing of the gammarids at the sampling sites cause the changes in population density.

\subsection{Environmental conditions}

Both life histories and microgeographic distribution are determined by degree of and variation in environmental factors. Natural processes as well as human activities like pollution constitute these environmental factors. For data on environmental factors see Goedmakers (1980).

In part I of this study (Goedmakers, 1980) a mathematical analysis showed the rather smooth transition in environmental conditions between the upper (stations 2 and 3 ) and middle (stations 6-12) reaches of the Slack. The animals, however, react sharply to a very small change in environmental conditions (fig. 60).

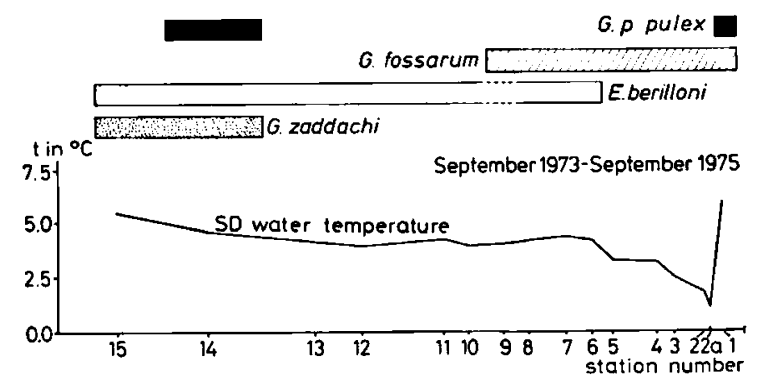

Fig. 60. Microgeographic variation in the standard deviation of water temperature (entire sampling period averaged) with the distribution patterns of $G$. p. pulex, G. fossarum, $E$ berilloni and $G$. zaddachi. (An uninterrupted bar means that a species is always present in percentages above $5 \%$; a broken bar means a sporadic occurrence above $5 \%$.) 

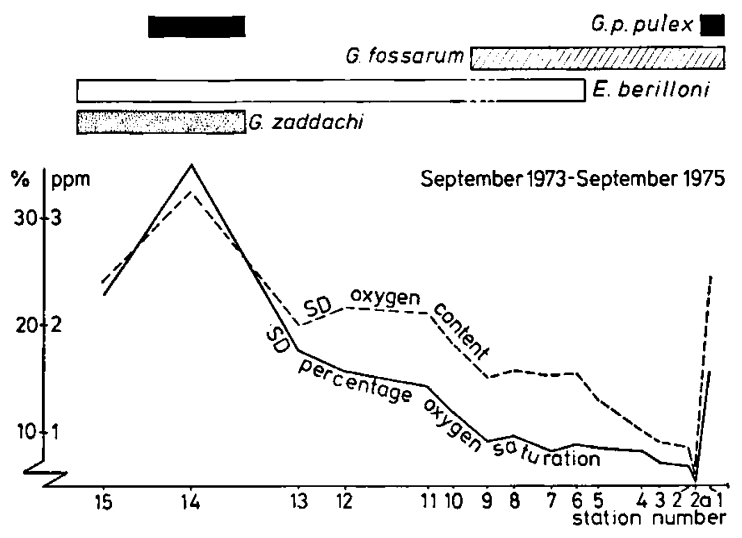

Fig. 61. Microgeographic variation in the standard deviation of the percentage of oxygen saturation of the water and of the oxygen content of the water (entire sampling period averaged) with the distribution patterns of $G$. p. pulex, $G$. fossarum, E. berilloni and $G$. zaddacbi. (An uninterrupted bar means that a species is always present in percentages above $5 \%$; a broken bar means a sporadic occurrence above $5 \%$.)

At stations where the fluctuation in water temperature exceeds a certain range, E. berilloni forms a substantial part of the gammarid population (fig. 60).

The influence of pollution is reflected in the variation in saturation with oxygen. A high level of variation in saturation of the water with oxygen (caused by pollution) means a boundary for the occurrence of G. fossarum (fig. 61).

Although rather high conductivity values (not caused by pollution) can be tolerated by $G$. fossarum (Goedmakers, 1980: fig. 19, station 2a), the increasing conductivity in the middle reaches of the Slack, with a relatively large range due to pollution, constitutes a boundary for $G$. fossarum. Above a certain level of and range in conductivity, this species occurs sporadically.

As we have seen in section 5.5, life histories are deeply influenced by temperature. Influence of other environmental factors on life histories will be less, since these other environmental factors never reach critical values in the river Slack.

\section{CONCLUSIONS}

Population density in the river Slack varies between 500 and 10,000 gammarids $\cdot \mathrm{m}^{-2}$. We found highest abundances in spring regions and head- waters. Local variation in population density was not caused by a sudden increase of the population after the hatching of juveniles. Changes in dispersion patterns probably caused the changes in abundances.

Microgeographic distribution of gammarids in the river Slack was rather stable from September 1973 to September 1975. The higher temperatures during the second sampling year were probably the cause of the small upstream shift of the centre of the distribution patterns of both $E$. berilloni and G. fossarum.

$G$. p. pulex lives in substantial percentages at stations 1 and 14 only. Station 1 is the spring region of the Slack with very unstable environmental conditions. Station 14 is situated in the freshwater tidal area of the river Slack. G. p. pulex is an euryecious species.

$G$. fossarum inhabits the upper, and part of the middle region of the Slack down to station 9. Its distribution area is most likely limited by pollution, with its effects on oxygen saturation and conductivity. $G$. fossarum is a cold stenothermous species, and rising water temperatures, for instance due to long hot summers, influence its distribution pattern seriously.

$E$. berilloni lives in the middle region of the Slack, from station 6 downwards, in the lower reaches and is even found in the estuarine part of the river. Its upper boundary coincides with a change in the range of water temperatures: $E$. berilloni occurs in the stretch where the variation in water temperature is considerably higher than more upstream. The almost absence of $E$. berilloni at station 9 is presumably caused by invasion of $G$. fossarum from a nearby springbrook after a period during which gammarids were absent from that station, while $E$. berilloni had to invade this site from places further away. It is a warm eurythermous species.

G. zaddachi inhabits the brackish part of the river Slack and invades the freshwater tidal area only certain times of the year.

Life histories of $E$. berilloni, $G$. fossarum and $G$. p. pulex differ to a large extent both inter- and intraspecifically. This diversity is evident in results on size, growth rates, sexual activity, population 
structure, sex ratio, fecundity and parasitation. Laboratory experiments on egg incubation time and reproduction success affirmed the interspecific dissimilarity and the important role of temperature on life processes.

E. berilloni is a species with a periodical way of reproducing in the Slack. Its sexual activity stops at low temperatures. A female produces one or two batches of juveniles. Its fecundity is rather high. The percentage of females is higher than that of males.

$G$. fossarum is a species with sometimes a perioaicai and at other times a constant way of reproducing. Its sexual activity is highest at for the river Slack common temperatures around $10^{\circ} \mathrm{C}$. A female produces more batches of juveniles (in most cases two) than an E. berilloni female, but fecundity is about twice as low. The mean percentage of males is about the same as that of females, but shows a large microgeographic variation.

Although we had only few data on $G . p$. pulex, we concluded that this species has a periodical way of reproducing in the Slack. Its sexual activity is highest around $15^{\circ} \mathrm{C}$, which is rather high for conditions in this river. A female produces one or two batches of offspring, with more eggs per batch than $G$. fossarum females. In the population at station 1 males dominate.

The large difference between our number of broods and the number, 6, Welton \& Clarke (1980) stipulated for G. p. pulex shows the hazards of an extrapolation of laboratory data to the field situation. The poor results of our laboratory experiments, especially for $E$. berilloni, made us cautious to use these data in prognoses about life cycles. Much more research is necessary to fit laboratory and field data together.

The microgeographic and intraspecific differences in life processes are shown in figs. 38, 39, $46,47,55,56$ and tables IV to XI. Reproducing animals seemed able to colonize stations outside the main distribution area of each species first.

Seasonal variation in microgeographic distribution did not exist. The normal, temperaturerelated fluctuations in size, growth rates, sexual activity and population structure were found. After a period of almost no reproduction, males seem to be produced first. Fecundity of females of a certain length appears to increase as water temperatures rise, and decrease later in the year.

The between-year differences are mainly caused by a higher mean temperature and a mild, although long cold period in the second year (1974-1975). Perhaps pollution increased during this year, although higher temperatures may simply have enlarged the influence of pollution. In general, sexual activity increased, sizes were larger and growth rates higher during the second year, while fecundity was smaller.

\section{REFERENCES}

Armitage, P. D., A. M. Machale \& D. C. Crisp, 1974. A survey of stream invertebrates in the Cow Green basin (Upper Teesdale) before inundation. Freshwat. Biol., 4: $369-398$.

Beld, F. A. J. van DEN, 1973. Cycles annuels de Gammarus duebeni duebeni Liljeborg, 1852, et de Gammarus pulex pulex (Linné, 1758) le long de la côte française du Boulonnais. Bull. zoöl. Mus. Univ. Amsterdam, 3 (13): 79-98.

BLom, H., unpubl. Verslag van de werkzaamheden tijdens de stageperiode van augustus 1978 tot februari 1979 aan het Instituut voor Taxonomische Zoölogie der Universiteit van Amsterdam: 1-58 (Stageverslag Instituut voor Taxonomische Zoölogie, Univ. Amsterdam, 1979).

Clemens, H. P., 1950. Life cycle and ecology of Gammarus fasciatus Say. Hydrobiol. Contr., Franz Theodore Stone Inst. Ohio Stat. Univ., 12: 1-61.

Dennert, H. G., A. L. Dennert, P. Kant, S. Pinkster \& J. H. STock, 1969. Upstream and downstream migrations in relation to the reproductive cycle and to envi- ronmental factors in the amphipod, Gammarus zaddachi. Bijdr. Dierk., 39: 11-43.

Frost, S., A. Huni \& W. E. Kershaw, 1971. Evaluation of a kicking technique for sampling stream bottom fauna. Can. J. Zool., 49 (2): 167-173.

Goedmakers, A., 1972. Gammarus fossarum Koch, 1835: Redescription based on neotype material and notes on its local variation (Crustacea, Amphipoda). Bijdr. Dierk., 42 (2) : 124-138.

- 1980 . Population dynamics of three gammarid species (Crustacea, Amphipoda) in a French chalk stream. Part I. General aspects and environmental factors. Bijdr. Dierk., 50 (1): 1-34.

Goedmakers, A. \& S. Pinkster, in press. Population dynamics of three gammarid species (Crustacea, Amphipoda) in a French chalk stream. Part III. Migration. Bijdr. Dierk., 51 (2).

Goedmakers, A. \& A. L. Roux, 1975. Essais d'hybridation entre plusieurs populations de Gammares du groupe pulex (Amphipoda). Crustaceana, 29 (1): 99-109. 
Heñze, K., 1932. Fortpflanzung und Brutpflege bei Gammarus pulex L. und Carinogammarus roeselii Gerv. Zool. Jahrb. (Allg. Zool.), 51: 398.441.

Hultin, L., 1971. Upstream movements of Gammarus pulex pulex (Amphipoda) in a South Swedish stream. Oikos, 22: 329-347.

HyNES, H. B. N., 1955. The reproductive cycle of some British freshwater Gammaridae. J. anim. Ecol., 4: 352-387.

- 1961 . The invertebrate fauna of a Welsh mountain stream. Arch. Hydrobiol., 57 (3): 344-388.

Iversen, T. \& J. JeSSEN, 1977. Life-cycle, drift and production of Gammarus pulex L. (Amphipoda) in a Danish spring. Freshwat. Biol., 7: 287-296.

Janssen, H., M. Scheepmaker, M. VAN CouwelaAr \& S. PINKSTER, 1979. Biology and distribution of Gamma. rus aequicauda and G. insensibilis (Crustacea, Amphipoda) in the lagoon system of Bages-Sigean (France). Bijdr. Dierk., 49 (1): 42-70.

Karaman, G. S. \& S. Pinkster, 1977. Freshwater Gammarus species from Europe, North Africa and adjacent regions of Asia (Crustacea-Amphipoda). Part I. Gam. marus pulex-group and related species. Bijdr. Dierk., 47 (1): 1-97.

Koch-Kallnbach, M. E. \& M. P. D. Meijering, 1977. Duration of instars and praecopulae in Gammarus pulex (Linnaeus, 1758) and Gammarus roeselii Gervais, 1835 under semi-natural conditions. Crustaceana, Suppl. 4: 120-127.

LehmanN, U., 1967. Drift und Populationsdynamik von Gammarus pulex fossarum Koch. Z. Morph. Ökol. Tiere, 60: 227.274.

LE Roux, M. L., 1933. Recherches sur la sexualité des Gammariens. Bull. biol. Fr. Belg., Suppl. 16: 1-138.

MACAN, T. T. \& C. MACKERETH, 1957. Notes on Gammarus pulex in the English Lake District. Hydrobiologia, 9 (1): 1-12.

Maitland, P. S., 1966. Notes on the biology of Gammarus pulex in the river Endrick. Hydrobiologia, 28: 142-152.

MAREN, M. J. VAN, 1977. Note on the occurrence of Acanthocephala larvae in gammaridean Amphipoda from the river Slack (France, Dépt. Pas-de-Calais). Bull. zoöl. Mus. Univ. Amsterdam, 6 (6): 45-48.

Meijering, M. P. D., 1972. Zur Methodik der Driftmessung im Rahmen produktionsbiologischer Untersuchungen in Fliessgewässern. Verh. dt. zool. Ges., 35: 69-73.

Meijering, M. P. D. \& W. Teichmann, 1978. Zeitpläne limnischer Gammariden unter naturnahen Bedingungen. Verh. Gesellsch. Ökol., Kiel, 7: 191-199.

MilleR, J. D. \& A. L. BuIKema, 1977. The effect of substrate on the distribution of the spring form (form III) of Gammarus minus Say, 1818. Crustaceana, Suppl. 4: $153-163$.

OBRDlík, P., 1972. A population of Rivulogammarus fossasarum Koch (Amphipoda) in a small fishless stream. Hydrobiologia, 40: 279-295.

Pieper, H., 1978. Ökophysiologische und produktionsbiologische Untersuchungen an Jugendstadien von Gammarus fossarum Koch 1835. Arch. Hydrobiol., Suppl. 54 (3): 257-327.
Pinkster, S., 1972. On members of the Gammarus pulexgroup (Crustacea-Amphipoda) from Western Europe. Bijdr. Dierk., 42 (2): 164-191.

—, 1973. The Echinogammarus berilloni-group, a number of predominantly Iberian amphipod species (Crustacea). Bijdr. Dierk., 43 (1): 1-39.

RAVENSTEIN, K., unpubl. Verband tussen koplengte en aantal eieren bij Gammarus fossarum en Echinogammarus berilloni: 1-15 (Stageverslag Instituut voor Taxonomische Zoölogie, Univ. Amsterdam, 1978).

REES, P., 1972. The distribution of the amphipod Gammarus pseudolimnaeus Bousfield as influenced by oxygen concentration, substratum, and current velocity. Trans. Am. microsc. Soc., 91 (4): 514-529.

Roux, A. L., 1970. Le cycle de reproduction de deux espèces étroitement parentes de Crustacés Amphipodes: Gammarus pulex et $G$. fossarum. Annls. Limnologie, 6 (1): 27.49.

__, 1971. Les Gammares du groupe pulex. Essai de systématique. II. Quelques caractéristiques écologiques et physiologiques. Archs. Zool. exp. gén., 112: 471-503.

-_, 1975. Température stable et température fluctuante. II. Etude comparative de leurs effets sur la durée d'intermue de Gammaridae femelles. Verh. int. Verein. theor. angew. Limnol., 19: 3014-3021.

Sexton, E. W., 1942. The relation of Gammarus zaddachi Sexton to some other species of Gammarus occurring in fresh, estuarine and marine waters. J. mar. biol. Ass. U.K., 25: 575-606.

Steenbergen, H., M. van Hezewijk \& F. Schuring, unpubl. Onderzoek naar de populatieopbouw en migratieactiviteit van gammariden in de Slack: 1-99 (Doctoraalverslag Instituut voor Taxonomische Zoölogie, Univ. Amsterdam, 1979)

Sukop, I., 1975. A population of Gammarus fossarum Koch (Amphipoda) in a karstic stream. Věst. čsl. Spol. zool., 39 (1): 73-79.

TeckelmanN, U., 1974. Temperaturwirkungen auf Wachstum und Stoffwechsel kaltstenothermer Fliesswassertiere. Arch. Hydrobiol., 74 (4): 479-527.

TeIXeIRA DE MATTOS, J., unpubl. De verspreiding van 4 soorten gammariden (Crustacea Amphipoda) en hun jaarcyclus in de rivier de Slack (Pas-de-Calais, Nrd. Frankrijk): 1.31 (Doctoraalverslag Instituut voor Taxonomische Zoölogie, Univ. Amsterdam, 1977).

VINCENT, M., 1971. Ecologie et écophysiologie des Gammaridés épigés du centre ouest. Thèse Fac. Sci. Univ. Limoges, 5313: i-iv, 1-132.

WEERD, W. DE, unpubl. Onderzoek naar twee verschillende monstermethodes: - elektrisch vissen, - monstername met een schepnet: 1-8 (Stageverslag Instituut voor Taxonomische Zoölogie, Univ. Amsterdam, 1980).

WeLton, J. S., 1979. Life-history and production of the amphipod Gammarus pulex in a Dorset chalk stream. Freshwat. Biol., 9: 263-275.

Welton, J. S. \& R. T. Clarke, 1980. Laboratory studies on the reproduction and growth of the amphipod Gammarus pulex. J. anim. Ecol., 49: 581-592. 